University of Nebraska - Lincoln

DigitalCommons@University of Nebraska - Lincoln

2001

Perspectives of Giant Magnetoresistance

Evgeny Y. Tsymbal

University of Oxford, tsymbal@unl.edu

David G. Pettifor

University of Oxford, david.pettifor@materials.ox.ac.uk

Follow this and additional works at: https://digitalcommons.unl.edu/physicstsymbal

Part of the Condensed Matter Physics Commons

Tsymbal, Evgeny Y. and Pettifor, David G., "Perspectives of Giant Magnetoresistance" (2001). Evgeny Tsymbal Publications. 50.

https://digitalcommons.unl.edu/physicstsymbal/50

This Article is brought to you for free and open access by the Research Papers in Physics and Astronomy at DigitalCommons@University of Nebraska - Lincoln. It has been accepted for inclusion in Evgeny Tsymbal Publications by an authorized administrator of DigitalCommons@University of Nebraska - Lincoln. 


\section{Perspectives of Giant Magnetoresistance}

E.Y.Tsymbal and D.G.Pettifor

Department of Materials, University of Oxford, Parks Road, Oxford OX1 3PH, UK

I. Introduction

II. Origin of GMR
1. Spin-dependent conductivity
2. Role of band structure
3. Resistor model

III. Experimental survey

4. Composition dependence

5. Nonmagnetic layer thickness dependence

6. Magnetic layer thickness dependence

7. Roughness dependence

8. Impurity dependence

9. Outer-boundary dependence

10. Temperature dependence

11. Angular dependence

IV. Free-electron and simple tight-binding models
12. Semiclassical theory
13. Quantum-mechanical theory
14. Tight-binding models

V. Multiband models
15. Ballistic limit
16. Semiclassical theory
17. Tight-binding models
18. First-principle models

\section{CPP GMR}

VII. Conclusions 


\section{INTRODUCTION}

Giant magnetoresistance (GMR) is one of the most fascinating discoveries in thin-film magnetism, which combines both tremendous technological potential and deep fundamental physics. Within a decade of GMR being discovered in 1988 commercial devices based on this phenomenon, such as hard-disk read-heads, magnetic field sensors and magnetic memory chips, had become available in the market. These achievements would not have been possible without a detailed understanding of the physics of GMR, which requires a quantum-mechanical insight into the electronic spin-dependent transport in magnetic structures.

The discovery of GMR was to a great extent due to the significant progress in thin-film deposition techniques, which made it possible to fabricate layers of various materials with nearly a monolayer precision. Thin films of a nanometer thickness can nowadays be routinely fabricated using molecular beam epitaxy (MBE), sputtering and electrodeposition. By stacking such thin films in multilayers one can create layered systems with properties, which are totally distinct from those of the constitutive bulk materials. Metallic magnetic multilayers, which consist of several ferromagnetic layers separated by non-magnetic layers, are very attractive as they exhibit a broad variety of unique electronic, magnetic, and transport properties.

Like other magnetoresistive effects, GMR is the change in electrical resistance in response to an applied magnetic field. It was discovered that the application of a magnetic field to a $\mathrm{Fe} / \mathrm{Cr}$ multilayer resulted in a significant reduction of the electrical resistance of the multilayer. ${ }^{1}$ This effect was found to be much larger than either ordinary or anisotropic magnetoresistance and was, therefore, calle "giant magnetoresistance" or GMR. A similar, though diminished effect was simultaneously discovered in $\mathrm{Fe} / \mathrm{Cr} / \mathrm{Fe}$ trilayers. ${ }^{2}$ As was shown later, high magnetoresistance values can also be obtained in other magnetic multilayers, such as $\mathrm{Co} / \mathrm{Cu}$. The change in the resistance of the multilayer arises when the applied field aligns the magnetic moments of the successive ferromagnetic layers, as is illustrated schematically in Fig.1. In the absence of the magnetic field the magnetizations of the ferromagnetic layers are antiparallel. Applying the magnetic field, which aligns the magnetic moments and saturates the magnetization of the multilayer, leads to a drop in the electrical resistance of the multilayer.

In order to observe GMR one has to provide an opportunity to reorient the magnetic moments of the ferromagnetic layers relative to one another. In magnetic multilayers this can be achieved due to the effect of antiferromagnetic interlayer coupling, ${ }^{3}$ which is a particular case of interlayer exchange coupling. The interlayer exchange coupling is mediated by the itinerant electrons in the metallic spacer layer and is an analogue of the Ruderman-Kittel-Kasuya-Yosida (RKKY) interaction between localized magnetic moments in a non-magnetic host metal. The interlayer exchange coupling oscillates between ferromagnetic and antiferromagnetic as a function of the thickness of the nonmagnetic layer, ${ }^{4}$ By choosing an appropriate thickness of the non-magnetic layer it is, therefore possible to create an antiparallel configuration of the ferromagnetic layers and then reorient (align) the moments by an applied magnetic field.

The presence of an antiferromagnetic interlayer coupling is not, however, a necessary condition for GMR to occur. Antiparallel alignment can also be obtained by introducing different coercivities of the successive ferromagnetic layers. ${ }^{5-7}$ In this case the magnetic moments of the soft and har magnetic layers switch at different values of the applied magnetic field providing a field range in which they are antiparallel and the resistance is higher. Another way to change the alignment of the magnetizations is to use a spin valve. ${ }^{8}$ In the spin valve the magnetization of one ferromagnetic laye is pinned by the exchange coupling with an adjacent antiferromagnetic layer, whereas the magnetization of the other ferromagnetic layer is free to rotate with the applied magnetic field. Although the measured values of GMR are higher in magnetic multilayers, spin valves are more the point of view of applications, because only small magnetic fiel applied to change the resistance. Magnetic granular solids represent another system, which displays the GMR effect. ${ }^{9}$ In these materials ferromagnetic precipitates are embedded in a non-magnetic host metal film. The randomly-oriented magnetic moments of the precipitates can be aligned by the applied magnetic field which results in a resistance drop. The various types of systems in which GMR is observed are shown in Fig.2.

a

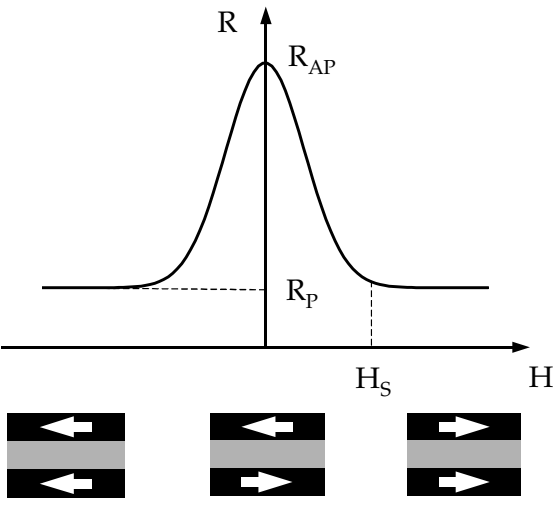

$\mathrm{M}$

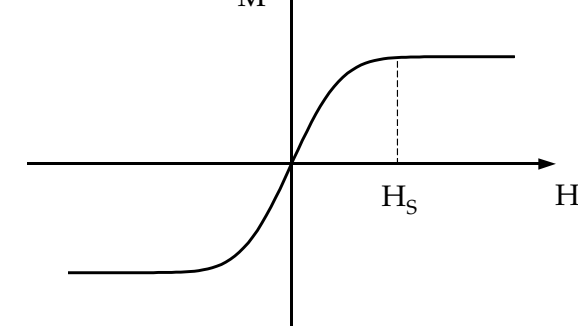

Fig.1 Schematic representation of the GMR effect. (a): Change in the resistance of the magnetic multilayer as a function of applied magnetic field. (b): The magnetization configurations (indicated by the arrows) of the multilayer (trilayer) at various magnetic fields: the magnetizations are aligned antiparallel at zero field, the magnetizations are aligned parallel magnetization curve for the multilayer.

GMR is distinguished from both ordinary magnetoresistance and anisotropic magnetoresistance (AMR) which are also present in layered and granular magnetic systems. Ordinary magnetoresistance arises from the effect of the Lorentz force on the electron trajectories due to the applied magnetic field. In contrast to GMR, it does not saturate at the saturation magnetic field and is usually small in metals (less than $1 \%$ in fields of the order of 1 Tesla). AMR originates from the spin-orbit interaction and causes the resistance to depend on the relative orientations of the magnetization and the electric current. The magnetic field range in which the AMR effect occurs is governed by the field needed to change the direction of the magnetic moment. For example, permalloy $\left(\mathrm{Ni}_{80} \mathrm{Fe}_{20}\right)$ films, which are presently employed in sensor applications, exhibit the AMR effect of $1-2 \%$, the resistance change taking place in a field range of a few Gauss. ${ }^{10}$ Contrary to anisotropic magnetoresistance, GMR arises 
due to the dependence of the resistivity in layered and granular magnetic structures on the loca magnetic configuration rather than on the orientation of the applied magnetic field with respect to the electric current.

a
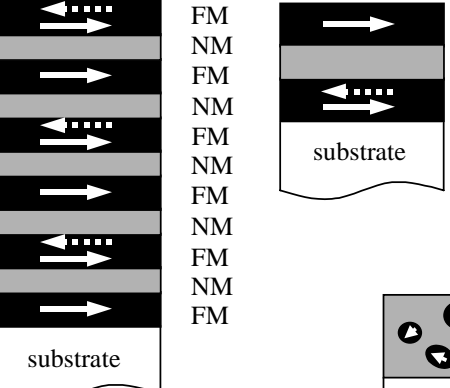

substrate

FM

NM

FM

FM

bstrate
FM - hard

NM

FM - soft

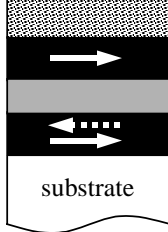

FM - pinned

NM

FM - free

d

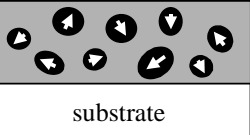

therefore the physics of GMR is related to spin-dependent electronic transport in complex metal systems. On the other hand, TMR is observed in layered systems where magnetic metallic layers are separated by an insulating spacer layer and is a consequence of spin-polarized tunneling.

Most experiments on GMR are performed by measuring the electric current in the plane of the multilayer, i.e. within the current-in-the-plane (CIP) geometry. This geometry is currently used for the industrial applications of GMR. Measuring the current perpendicular to the multilayer plane, i.e. within the current-perpendicular-to-the-plane (CPP) geometry, is much more difficult. This is due to the very small thickness of the multilayer and consequently the very low CPP resistance, which is not easy to detect. There are several ways to solve this problem, one of which is to perform the experiments using superconducting contacts. ${ }^{13}$ Although this technique has the advantage of relatively simple sample preparation, measurements can be performed only at low temperatures. Other techniques, which avoid this problem, are based on lithographically defined pillar structures ${ }^{14}$ on growing the magnetic multilayers on prestructured (grooved) substrates $^{15,16}$ (Fig.3a) or on electrodeposition of the multilayer nanowires into the pores of an insulating polymer matrix ${ }^{17,1}$ (Fig.3b). CPP GMR appeared to be very attractive, because its magnitude is higher than the corresponding magnitude of CIP GMR. In addition we will see that CPP experiments provide important information about the mechanisms of giant magnetoresistance.

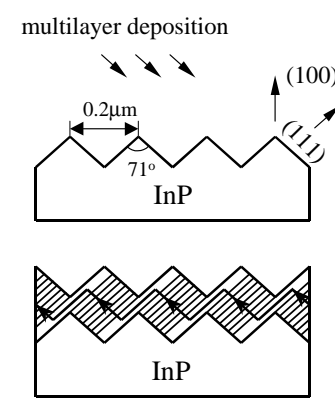

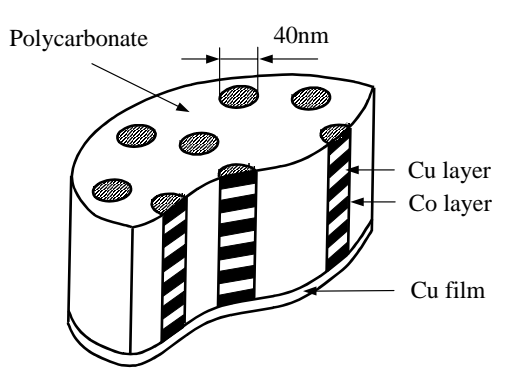

Fig.3 Techniques for current-perpendicular-to-the-plane (CPP) GMR, which can be used at room temperature: grooved InP substrate and a multilayer evaporated at an angle to the surface. Current flow
gromention grooved InP substrate and a multilayer evaporated at an angle to the suftace. Current flow
is indicated by the arrows. After Gijs $e t$ al. $^{16}$ (b): Schematic representation of the array of multilayer nanowires in an insulating polymer matrix. After Piraux et $a l^{18}$

Since the discovery of GMR the theoretical treatment of this effect became the subject of much attention. First theories of GMR were based mainly on free-electron models. Although these theorie are useful for the qualitative understanding of electronic transport in magnetic multilayers and can provide valuable insight into the phenomenon, they cannot be applied to the quantitative treatment of GMR, due to the complex spin-polarized electronic structure of the magnetic multilayers. It is wellknown that the band structures of transition metal ferromagnets which are mostly used in the GMR structures are characterized by unfilled $d$ bands which can not be described by a single parabolic band at the Fermi energy. Recent advances in electronic transport and band structure theory have made it possible to develop realistic multiband models and perform first-principle calculations of GMR. 
These models provided new conceptual insights into the phenomenon and have extended our fundamental understanding of GMR.

Reviews on GMR have been published by Fert and Bruno, ${ }^{19}$ Levy $^{20}$ and Dieny ${ }^{21}$ covering the field upto 1994. Other reviews by Gijs and Bauer, ${ }^{22}$ Ansermet, ${ }^{23}$ Bass and Pratt,${ }^{24}$ Fert and Piraux and Gijs ${ }^{26}$ are devoted specifically to the CPP GMR. Very recently two review papers by Coehoorn ${ }^{27}$ and by Barthelemy et al. ${ }^{28}$ have appeared. The first one highlights the theoretical and experimental results, which are of particular interest for applications of spin valves in read heads. The second one discusses the nature of GMR by accenting the importance of CPP geometry and gives a full list of experimental papers.

The present review is devoted to the physics of giant magnetoresistance. We emphasize the role of the spin-polarized electronic band structure, which is crucial for understanding GMR. In section II, the origin of GMR is explained and a simple resistor model is introduced. In section III, we overview the experimental data on CIP GMR in magnetic multilayers and spin valves and discuss the dependence of GMR on composition, layer thickness, roughness, impurities, outer boundaries and temperature. The theoretical formulations of GMR within free-electron and simple tight-binding models are reviewed in section IV both from the semiclassical and quantum mechanical viewpoints. Multiband models for GMR are reviewed in section V. Starting from the ballistic regime of conduction, we discuss both the semiclassical and quantum mechanical approaches to GMR within the diffusive limit. The mechanisms, which are responsible for GMR, are discussed and the interpretation of selected experimental results is presented. A separate section VI is devoted to CPP GMR, which has recently attracted much attention due to new experimental and theoretical results. In conclusion, we indicate directions for future work on GMR.

\section{ORIGIN OF GMR}

GMR can be qualitatively understood using the Mott model, which was introduced as early as 1936 to explain the sudden increase in resistivity of ferromagnetic metals as they are heated above the Curie temperature. ${ }^{29}$ There are two main points proposed by Mott. First, the electrical conductivity in metals can be described in terms of two largely independent conducting channels, corresponding to the up-spin and down-spin electrons, which are distinguished according to the projection of their spins along the quantization axis. The probability of spin-flip scattering processes in metals is normally small as compared to the probability of the scattering processes in which the spin is conserved. This means that the up-spin and down-spin electrons do not mix over long distances and, therefore, the electrical conduction occurs in parallel for the two spin channels. Second, in ferromagnetic metals the scattering rates of the up-spin and down-spin electrons are quite different, whatever the nature of the scattering centers is. According to Mott, the electric current is primarily carried by electrons from the valence $s p$ bands due to their low effective mass and high mobility. The $d$ bands play an important role in providing final states for the scattering of the $s p$ electrons. In $d$ bands play an important role in providing final states for the scattering of the $s p$ electrons.
ferromagnets the $d$ bands are exchange-split, so that the density of states is not the same for the upspin and down-spin electrons at the Fermi energy. The probability of scattering into these states is proportional to their density, so that the scattering rates are spin-dependent, i.e. are different for the two conduction channels. Although, as we will see below, this picture is too simplified in a view of the strong hybridization between the $s p$ and $d$ states, it forms a useful basis for a qualitative understanding of the spin-dependent conduction in transition metals.

Using Mott's arguments it is straightforward to explain GMR in magnetic multilayers. We consider collinear magnetic configurations, as is shown in Fig.4, and assume that the scattering is strong for electrons with spin antiparallel to the magnetization direction, and is weak for electron with spin parallel to the magnetization direction. This is supposed to reflect the asymmetry in the density of states at the Fermi level, in accordance with Mott's second argument. For the parallel- aligned magnetic layers (the top panel in Fig.4a), the up-spin electrons pass through the structure almost without scattering, because their spin is parallel to the magnetization of the layers. On the contrary, the down-spin electrons are scattered strongly within both ferromagnetic layers, because their spin is antiparallel to the magnetization of the layers. Since conduction occurs in parallel for the two spin channels, the total resistivity of the multilayer is determined mainly by the highly-conductive up-spin electrons and appears to be low. For the antiparallel-aligned multilayer (the top panel in Fig.4b), both the up-spin and down-spin electrons are scattered strongly within one of the ferromagnetic layers, because within the one of the layers the spin is antiparallel to the magnetization direction. Therefore, in this case the total resistivity of the multilayer is high.

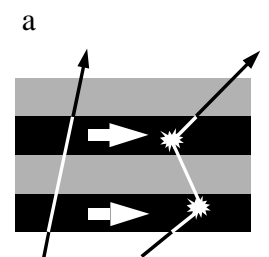

up spin down spin

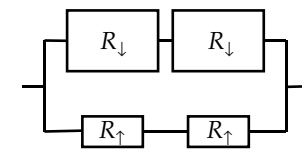

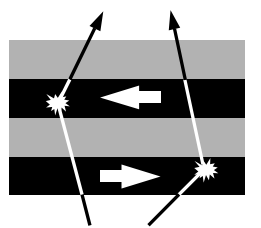

up spin down spin

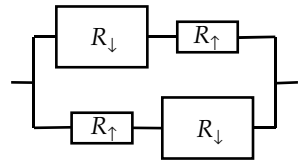

Fig.4 Schematic illustration of electron transport in a multilayer for parallel (a) and antiparalle (b) magnetizations of the successive ferromagnetic layers. The magnetization directions are indicated by the arrows. The solid lines are individual electron trajectories within the two spin channels. It is assumed that the mean free path is much longer than the layer thicknesses and the net electric current flows in the plane of the layers. Bottom panels show the resistor network within the two-current series resistor model. For the parallel-aligned multilayer (a), the up-spin electrons pass through the structure almost without scattering, whereas the down-spin electrons are scattered strongly within both ferromagnetic layers. multilayer is low. For the antiparallel-aligned multilayer (b), both the up-spin and downspin electrons are scattered strongly within one of the ferromagnetic layers, and the tot resistivity of the multilayer is high.

The same arguments can be used for understanding GMR in granular materials. In the absence of a magnetic field, the magnetic moments of the ferromagnetic granules are randomly-oriented. Thi implies that both up- and down-spin electrons are scattered strongly by the granules, the magnetic moments of which are close to antiparallel. The resistance in this case is large. When a saturating magnetic field is applied, the magnetic moments are aligned and the resistance is low, like in the case of the parallel-aligned multilayer.

Therefore, as was originally suggested by Baibich et al., ${ }^{1}$ spin-dependent scattering is the primary origin of GMR. An understanding of the microscopic mechanisms, which cause spin-dependent scattering in magnetic systems, is one of the most important questions, which this review attempts to answer. In addition, we will see that there are other mechanisms distinct from spin-dependent 
scattering which are important for understanding GMR and which will also be addressed in this review. We start now from a qualitative picture for the spin-dependent conduction in ferromagnets.

\section{Spin-dependent conduction}

According to Mott's first argument, the conductivity of a metal is the sum of the independent conductivities for the up-spin and down-spin electrons:

$\sigma=\sigma_{\uparrow}+\sigma_{\downarrow}$

Within each conduction channel the conductivity is determined by various factors. In order to illustrate their role we use the Drude formula (e.g., ref.30) which can be expressed as follows:

$$
\sigma_{\text {Drude }}=\frac{e^{2}}{\pi \hbar} \frac{k_{F}^{2}}{6 \pi} \lambda \text {. }
$$

Here $\sigma_{\text {Drude }}$ is the Drude conductivity per spin, $e^{2} / \pi \hbar \approx 0.387 \cdot 10^{-4} \Omega^{-1}$ is the spin conductance quantum, $k_{F}$ is the Fermi momentum, and $\lambda$ is the mean free path, which is the product of the relaxation time $\tau$ and the Fermi velocity $\mathrm{v}_{F}$, i.e.

$\lambda=\mathrm{v}_{F} \tau$.

We do not display explicitly the spin indices here - it is assumed that all the above quantities are in general spin-dependent. Although the Drude formula is valid only for free electrons, it is useful to understand qualitatively the factors affecting the spin-dependent conductivity.

The conductivity is determined by the electrons which have the Fermi energy. Due to the Paul exclusion principle the electrons which lie below the Fermi level can not gain energy responding to the small applied electric field, because all the states at higher energies are occupied. As a consequence, only electrons at the Fermi level can contribute to the electric current. As can be seen from Eq.(1.2), the conductivity is proportional to the cross sectional area of the Fermi surface $\sim k_{F}^{2}$, which characterizes the number of electrons contributing to the conduction. The mean free path depends of the Fermi velocity and the relaxation time, the latter can be estimated from the Fermi golden rule

$$
\tau^{-1}=\frac{2 \pi}{\hbar}\left\langle V_{\text {scat }}{ }^{2}\right\rangle n\left(E_{F}\right) .
$$

Here $\left\langle V_{\text {scat }}{ }^{2}\right\rangle$ is an average value of the scattering potential and $n\left(E_{F}\right)$ is the density of electronic states at the Fermi energy $E_{F}$ for the appropriate spin.

Although all the quantities which enter expressions (1.2)-(1.4) are in general spin-dependent, the origin of the spin dependence is different. The Fermi momentum $k_{F}$ and the Fermi velocity $\mathrm{v}_{F}$ are intrinsic properties of the metal and entirely determined by the electronic band structure of the metal. In ferromagnetic metals these quantities are different for the up- and down-spin electrons. The density of states at the Fermi energy $n\left(E_{F}\right)$ is also determined by the spin-polarized band structure. It is the density of states, which was referred to by Mott, arguing that the scattering rates in ferromagnetic metals are spin-dependent.

On the contrary, the scattering potential which enters Eq.(1.4) is not an intrinsic property of the metal. It is generated by the scatterers such as defects, impurities, or lattice vibrations. The scattering potential can be either spin-dependent or spin-independent, which is determined by the particular mechanism of scattering. For example, spin-dependent scattering potentials produced by impurities in dilute magnetic alloys leads to the spin asymmetry of the conductivity in these alloys. ${ }^{31-33}$ This has to be taken into account when treating GMR in magnetic layered systems in which ferromagnetic layers are often alloys such as permalloy $\mathrm{Ni}_{80} \mathrm{Fe}_{20}$. Spin-dependent scattering potentials might also contribute to GMR at the interfaces between ferromagnetic and non-magnetic layers. In real magnetic multilayers these interfaces are not ideal. Interfacial roughness and/or substitutional disorder (i.e. mixing of the adjacent metal atoms at the interface) are always present in experiments. Randomness of the atomic potentials at the interface results in enhanced interfacial scattering. If for one spin orientation the atomic potentials of the magnetic and nonmagnetic atoms are similar but for the other spin orientation they are dissimilar, then one would expect a strong spin-dependent scattering due to spin orientation they are dissimilar, then one
the spin dependence in the scattering potential.

The relative importance of spin-dependent scattering potentials can, however, be diminished in real GMR structures which are far from being perfect. Various types of defects such as grain boundaries, stacking faults and misfit dislocations are always present in the multilayers. Because the relaxation time in Eq.(1.4) is determined by the configurationally-averaged value of the scattering potential squared, various types of scattering centers can make this average value spin-independent. In these circumstances the spin-polarized band structure of the multilayer becomes decisive and usually gives the dominant contribution to the spin dependence of the mean free path and the conductivity.

\section{Role of band structure}

The electronic band structure of the multilayer is probably the most important property which determines the spin-dependent conduction and consequently is responsible for the GMR. In most experiments on GMR the ferromagnetic $3 d$ transition metals $\mathrm{Co}, \mathrm{Fe}$ and $\mathrm{Ni}$, and their alloys, such as permalloy $\mathrm{Ni}_{80} \mathrm{Fe}_{20}$, are used in combination with non-magnetic spacer layers, such as $\mathrm{Cr}$ or the noble metals $\mathrm{Cu}, \mathrm{Ag}$ and $\mathrm{Au}$. The electronic band structure of these metals is characterized by a number of similar features which we discuss below.

Due to the spin-orbit coupling of the $3 d$ transition metals being very weak the electronic structure for the up-spin and down-spin electrons can be considered independently. The $3 d$ elements are characterized by the presence of the $4 s, 4 p$ and $3 d$ valence states, which are distinguished by their orbital momentum. The $4 s$ and $4 p$ states create a dispersive $s p$ band which is similar to a free-electron band. The $s p$ electrons have a high velocity, a low density of states and consequently a long mean free path, i.e. they may be thought to be mainly responsible for the conductivity in $3 d$ metals. On the contrary, the $d$ band is localized in a relatively narrow energy window and is characterized by a high density of states and a low velocity of electrons. In the interval of energy where the $s p$ and $d$ bands cross, they can not be considered as independent because of the strong $s p-d$ hybridization, which modifies substantially the band structure. It changes dramatically the properties of the $s p$ electrons, which is reflected in the band bending and results in a reduced velocity associated with the $s p$ band These features are evident from Fig.5a, in which the electronic band structure of $\mathrm{Cu}$ is shown.

In ferromagnetic $3 d$ metals the $d$ band is exchange-split. Due to the localized nature of the $d$ electrons, two $d$ electrons experience a strong Coulomb repulsion provided that they have antiparallel spins and occupy the same orbital. To reduce the energy it is advantageous for the $d$ electrons to have parallel-oriented spins, because the Pauli exclusion principle does not permit two electrons with the same spin to approach each other closely (i.e. occupy the same orbital) and hence the Coulomb interaction is reduced. Therefore, the Coulomb repulsion in conjunction with the Pauli principle leads to the ferromagnetic exchange interaction and favors the formation of a spontaneous magnetic moment. However, putting all the electrons into states with the same spin direction increases the tota kinetic energy, the increase being larger the wider the $d$ band or lower the $d$-band density of states. There are, therefore, two competing tendencies, which have to be balanced in order to find whethe ferromagnetic ordering is favored. The condition which has to be satisfied for the appearance of ferromagnetism is the famous Stoner criterion $J n\left(E_{F}\right)>1$, where $J$ is the exchange constant (which takes the value of about $1 \mathrm{eV}$ for $3 d$ transition metals) and $n\left(E_{F}\right)$ is the density of states for a given spin at the Fermi energy. ${ }^{34}$ The Stoner criterion is satisfied for $b c c \mathrm{Fe}, f_{c c} \mathrm{Co}$ and $f c c \mathrm{Ni}$. Due to the exchange splitting of the $d$ bands, the number of occupied states is different for the up-spin and down- 
spin electrons, giving rise to the non-zero magnetic moments of $2.2 \mu_{\mathrm{B}}, 1.7 \mu_{\mathrm{B}}$ and $0.6 \mu_{\mathrm{B}}$ for Fe, Co and $\mathrm{Ni}$ respectively. In order to distinguish between the high and low-occupied spin states, the terms 'majority-spin electrons' and 'minority-spin electrons' are usually used. The band structure of Co as a representative of the ferromagnetic $3 d$ metals is shown in Figs.5b,c.

a
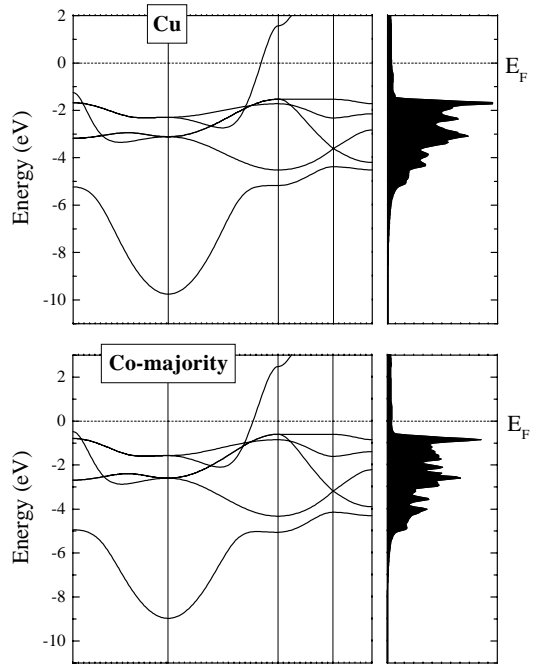

$\mathrm{c}$

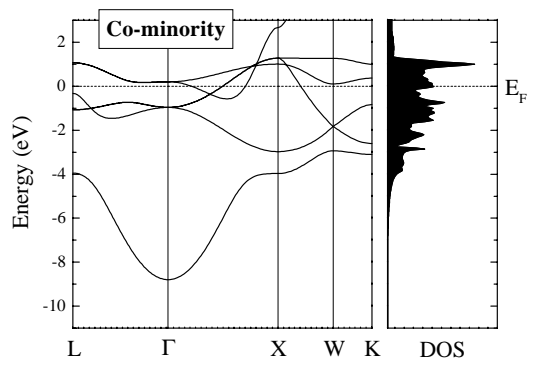

Fig.5 Electronic band structures (left panels) and the density of states (right panels) of $\mathrm{Cu}$ (a) and fcc Co for the majority-spin (b) and minority-spin (c) electrons. The band structure of non-magnetic $\mathrm{Cu}$ is same for the up-spin and down-spin electrons. It is characterized by the fully occupied $d$ bands and the presence of a dispersive $s p$ band at the Fermi energy, which result in high conductivity of $\mathrm{Cu}$. The electronic structure of ferromagnetic Co is different for the two spin orientations and is characterized by the exchange-split $d$ bands. The Fermi level lies within the $s p$ band for the majority-spin electrons, which leads to high conductivity of majority-spin channel. The Fermi level lies, however, within the $d$ band for the minority-spin electrons resulting in low conductivity of the minority-spin channel. In the latter case the $s p$ electrons are strongly hybridized with the $d$ electrons, which diminishes their contribution to conduction.

The conductivity is determined by the position of the Fermi energy with respect to the $d$ bands. In the case of $\mathrm{Cu}$, the $d$ bands are fully occupied and the Fermi level lies within the $s p$ band (Fig.5a). Due to the high velocity of the electrons within the $s p$ band and the low density of states with resultant low probability of scattering, the mean free path is long and $\mathrm{Cu}$ is a very good conductor. This is also the case for the other noble metals $\mathrm{Ag}$ and $\mathrm{Au}$. On the other hand, in the case of a ferromagnetic metal like $\mathrm{Co}$, as a result of the exchange splitting, the majority $d$ band is fully occupied, whereas the $d$ minority band is only partly occupied (Fig.5b,c). The Fermi level lies, therefore, within the $s p$ band for the majority spins but within the $d$ band for the minority spins. The exchange splitting of the spin bands leads to a crucial difference in the conductivity between the majority- and minority-spin electrons. For the majority spins the situation is similar to that in $\mathrm{Cu}$ : the conductivity is governed by the $s p$ electrons and is high. On the contrary, the conductivity of the minority-spin electrons is not entirely determined by the $s p$ electrons. Due to the strong $s p-d$ hybridization which mixes the $s p$ and $d$ states the contributions of both the $s p$ and $d$ electrons become important. The minority bands represent hybridized $s p d$ bands which are not dispersive and have a high density of states. This makes the mean free path associated with these bands relatively short and the minority-spin conductivity low, despite a sizeable factor proportional to the area of the multiban Fermi surface. These arguments, which are based on the spin-polarized band structure, explain the strong spin asymmetry in the conductivity of bulk Co.

The presence of the interfaces in a magnetic multilayer adds a new important feature to our discussion above of spin-dependent transport in bulk elemental ferromagnets. The two adjacent metals creating the interface have different band structures, which lead to a potential step at the interface and results in the transmission probability across the interface being less than 1 . If the interface separates ferromagnetic and non-magnetic metals the transmission will be spin-dependent due to the spin dependence of the band structure of the ferromagnetic layer. This can be illustrated by considering the band structures of $\mathrm{Co}$ and $\mathrm{Cu}$, which are shown in Fig.5. As is seen by comparing Fig.5a and $5 \mathrm{~b}$, the band structure of $\mathrm{Cu}$ is similar to the band structure of the majority spins in $\mathrm{Co}$ This good band matching implies a high transmission for the majority-spin electrons across the $\mathrm{Co} / \mathrm{Cu}$ interface. On the contrary, as is seen from Fig.5a and $5 \mathrm{c}$, there is a relatively large band mismatch between $\mathrm{Cu}$ and the minority spins in Co and consequently the transmission of the minority-spin electrons across the $\mathrm{Co} / \mathrm{Cu}$ interface is expected to be poor. Therefore, the interfaces of the $\mathrm{Co} / \mathrm{Cu}$ multilayer act as spin-filters. When the filters are aligned, the majority spin-electrons can pass through relatively easily. When the filters are antialigned, the electrons in both spin channels are reflected a one of the interfaces. This spin-dependent transmission is an important ingredient of the electronic transport in GMR structures.

Band matching also plays an important role in the spin-dependent interface scattering due to the intermixing of atoms near the interfaces. If we ignore the change in the chemical state of the atoms, i.e. assume that their atomic energy levels and magnetic moments are identical to those in the bulk of the adjacent layers, then the intermixing at the interface produces a random potential which is strongly spin-dependent. This spin dependence is a direct consequence of the good band matching for the majority spins in $\mathrm{Co} / \mathrm{Cu}$, which implies a small scattering potential, and the poor band matching for the minority spins in $\mathrm{Co} / \mathrm{Cu}$, which implies a large scattering potential. A similar behavior takes place in $\mathrm{Fe} / \mathrm{Cr}$ multilayers, where a very small scattering potential (good band matching) is expected for the minority-spin electrons, but a large scattering potential (bad band matching) is expected for the majority-spin electrons. Thus, the matching or mismatching of the bands between the ferromagnetic and nonmagnetic metals results in spin-dependent scattering potentials at disordered interfaces, which can contribute to GMR

\section{Resistor mode}

Physical insight into the origin of the current-in-the-plane (CIP) GMR can be obtained using the very simple resistor model. ${ }^{35,36}$ Although this model is not able to provide a quantitative description of the CIP GMR, it is useful as a starting point for understanding this phenomenon. 
According to the resistor model each metallic layer (and each interface) is treated as an independent resistor. Within each spin conduction channel the resistors are added in parallel or in series depending on the relationship between the mean free path and the layer thickness. If the mean free path is short compared to the layer thickness, then each layer conducts the electric curren independently and the resistors should be added in parallel. It is obvious that under this circumstance the resistance of the parallel and antiparallel configurations are the same and consequently the GMR is zero. The above observation indicates that for obtaining non-zero GMR the mean free path should be sufficiently long. This is consistent with the qualitative picture of GMR (the top panels of Fig.4) which is based on the possibility for the electrons to propagate across the spacer layer freely, sensing the magnetizations of the two consecutive ferromagnetic layers. In the limit the long mean free path being long compared to the layer thickness, the probability of scattering within the multilayer is the sum of scattering probabilities within each layer and each interface.$^{37}$ Therefore, within a given spin channel the total resistance is the sum of resistances of each layer and each interface, i.e. the resistors are connected in series. This limiting case is more relevant to the magnetic multilayers exhibiting giant magnetoresistance.

In order to build up the resistor network for the multilayer, we consider a unit cell which consisting of the four layers, two ferromagnetic and two non-magnetic, as is shown in the top panels of Fig.4a and 4b. We choose the global spin-quantization axis collinear to the magnetization directions. Within each ferromagnetic layer the electron spin can be either parallel or antiparallel to the magnetization direction. In the former case the electron is locally a majority-spin electron and in the latter case a minority-spin electron. The majority- and minority-spin resistivities of the ferromagnetic layer are different and are equal to $\rho_{\uparrow}$ and $\rho_{\downarrow}$ respectively. The resistance of the bilayer, which consists of the ferromagnetic layer and the spacer layer, for either of the two spin channels is equal to

$$
R_{\uparrow, \downarrow}=\rho_{N M} d_{N M}+\rho_{\uparrow, \downarrow} d_{F M},
$$

where $\rho_{N M}$ and $d_{N M}$ denote the resistivity and the thickness of the non-magnetic spacer layer and $t_{F M}$ is the thickness of the ferromagnetic layer. For simplicity the interface resistance between the ferromagnetic and spacer layers has been omitted. Using the resistances which are defined by Eq.(3.1) the equivalent network of resistors for the parallel and antiparallel magnetizations are shown in the bottom panels of Figs.4a and $4 \mathrm{~b}$. The total resistance of the parallel-aligned multilayer is then given by

$$
R_{P}=N \frac{R_{\uparrow} R_{\downarrow}}{R_{\uparrow}+R_{\downarrow}}
$$

where $N$ is the number of the four-layer unit cells within the multilayer. The total resistance of the antiparallel-aligned multilayer equals to

$$
R_{A P}=N \frac{R_{\uparrow}+R_{\downarrow}}{2} .
$$

Thus, the magnetoresistance ratio is determined by the simple expression

$$
\frac{\Delta R}{R}=\frac{R_{A P}-R_{P}}{R_{P}}=\frac{\left(R_{\downarrow}-R_{\uparrow}\right)^{2}}{4 R_{\downarrow} R_{\uparrow}} .
$$

Note that we use a definition in which GMR is normalized to the low resistance $R_{P}$. Although within this definition the GMR can be larger than $100 \%$, this definition is used in most papers devoted to GMR, and therefore we adopt it in this review.
Using Eqs. (3.1) and (3.4), it is easy to pinpoint the main factors which determine GMR. Let us first assume that the resistance of the spacer layer is small as compared to the resistance of the ferromagnetic layers. In this limit the expression for GMR is

$$
\frac{\Delta R}{R}=\frac{\left(\rho_{\downarrow}-\rho_{\uparrow}\right)^{2}}{4 \rho_{\downarrow} \rho_{\uparrow}}=\frac{(\alpha-1)^{2}}{4 \alpha},
$$

where the spin asymmetry parameter is defined by $\alpha=\rho_{\downarrow} / \rho_{\uparrow}$. As is obvious from Eq. (3.5), the magnitude of GMR is strongly dependent on the asymmetry in the resistivity between the two spin conduction channels in ferromagnetic layers. Large asymmetry, i.e. $\alpha>>1$ or $\alpha<<1$, is an importan requirement for obtaining high values of GMR. If there is no spin asymmetry in the resistivity of the two spin channels, i.e. $\alpha=1$, then the GMR will be zero.

Now we use Eq.(3.5) to make an estimate of $\mathrm{GMR}$ in $\mathrm{Co} / \mathrm{Cu}$ and $\mathrm{Fe} / \mathrm{Cr}$ multilayers. If we assume that $\alpha$ is entirely determined by the spin asymmetry in the scattering rates in Eq.(1.4), due to the spindependent density of states at the Fermi energy, we find $\alpha \approx 7$ for Co and $\alpha \approx 3$ for Fe. For the $\mathrm{Co} / \mathrm{Cu}$ multilayer this leads to a GMR value of $130 \%$, which is very close to the best published experimental result of $120 \%{ }^{38}$ However, for $\mathrm{Fe} / \mathrm{Cr}$ Eq. (3.5) gives a GMR of 30\%, which is far below the highest ever observed value of $220 \%{ }^{39}$ This is not surprising because the above model is too simplified as GMR depends on many other factors such as the properties of the FM/NM interface which were ignored in this estimate.

The finite resistance of the spacer layer may also be taken into account, which leads to

$\frac{\Delta R}{R}=$ $(\alpha-1)^{2}$

$\frac{R}{4\left(\alpha+p d_{N M} / d_{F M}\right)\left(1+p d_{N M} / d_{F M}\right)}$

where $p=\rho_{v M} / \rho_{\uparrow}$. Hence, for a given value of $\alpha$, the GMR will increase with decreasing $p d_{N M} / d_{F M}$. Therefore, in order to obtain higher GMR, it is important to have a low resistivity of the non-magnetic spacer layer. As a function of the spacer thickness $d_{N M}$, the GMR decrease monotonically and at large spacer thickness it falls off as $1 / d^{2}$. Although the drop in GMR with increasing spacer thickness is also found in experiments, the actual dependence on $d_{N M}$ is differen compared to this simplified model. As discussed later, the CIP GMR is found to decrease exponentially with $d_{N M}$ for large spacer thicknesses. The reason for this disagreement is that the series-resistor model is not applicable for $d_{N M}$ large compared to the mean free path. In the latter case, more sophisticated models have to be applied.

The series-resistor model is able to account for the inverse GMR effect. ${ }^{40}$ Eq.(3.5) suggests that the resistance of the parallel configuration is always smaller than the resistance of the antiparallel configuration. In most cases this statement is correct. However, there are exceptions. This can be seen, by considering a multilayer, which comprises different ferromagnetic layers. As is easy to show, in this case the GMR equals to

$$
\frac{\Delta R}{R}=\frac{\left(\alpha_{1}-1\right)\left(\alpha_{2}-1\right)}{\alpha_{1}(1+q)+\alpha_{2}\left(1+q^{-1}\right)},
$$

where $\alpha_{1}$ and $\alpha_{2}$ are the asymmetry parameters for the two different ferromagnetic layers, i.e. $\alpha_{1}=\rho_{\downarrow}^{(1)} / \rho_{\uparrow}^{(1)}$ and $\alpha_{2}=\rho_{\downarrow}^{(2)} / \rho_{\uparrow}^{(2)}$, and $q$ is the ratio of the up-spin resistivities in the two ferromagnets, i.e. $q=\rho_{\uparrow}^{(1)} / \rho_{\uparrow}^{(2)}$. It is clear from Eq.(3.7) that in the case when the two ferromagnetic layers have different asymmetries in resistivity, i.e. $\alpha_{1}>1$ and $\alpha_{2}<1$ or vice versa, then one can expect an inverse GMR.

The series-resistor model can be readily generalized to include spin-dependent interface resistances, by adding additional resistors in the network. As will be discussed in section VI, this 
model is a better approximation for the description of GMR within the CPP geometry, ${ }^{41-43}$ rather than the CIP geometry discussed here. For this reason it has often been used to obtain values of the spindependent bulk and interface resistances from CPP experimental data.

\section{EXPERIMENTAL SURVEY}

In this section we overview the main experimental results on GMR. Where it is not specified, we discuss the current-in-the-plane (CIP) geometry. A separate section VI will be devoted to GMR within the current-perpendicular-to-the-plane (CPP) geometry.

Giant magnetoresistance was discovered in 1988 by the group of Albert Fert on $\mathrm{Fe} / \mathrm{Cr}$ magnetic multilayers ${ }^{1}$ and the group of Peter Grünberg on $\mathrm{Fe} / \mathrm{Cr} / \mathrm{Fe}$ trilayers. ${ }^{2}$ In both cases the samples were grown using MBE and had [001] orientation of the layers. The $\mathrm{Cr}$ spacer layers were about $1 \mathrm{~nm}$ thick, so that the Fe layers were coupled antiferromagnetically providing an antiparallel alignment of their magnetizations at zero applied magnetic field. As the applied field is increased, the magnetic moments of the ferromagnetic layers progressively rotate towards the field, leading to a decrease in the resistance of the multilayer (trilayer). At saturation the magnetizations end up in a configuration of parallel alignment with the lowest value of the resistance. Fig.6 shows the variation in the resistance of the $\mathrm{Fe} / \mathrm{Cr}$ multilayer measured by Baibich et al. ${ }^{1}$ The highest magnitude of GMR in these experiments was found of $79 \%$ (using the definition of GMR given by formula (2.7)) at $\mathrm{T}=4.2 \mathrm{~K}$. The experiments was found of $79 \%$ (using the definition of GMR given by formula $(2.7)$ ) at $\mathrm{T}=4.2 \mathrm{~K}$. The
GMR effect was ascribed to the spin-dependent transmission of the conduction electrons between the $\mathrm{Fe}$ and $\mathrm{Cr}$ layers.

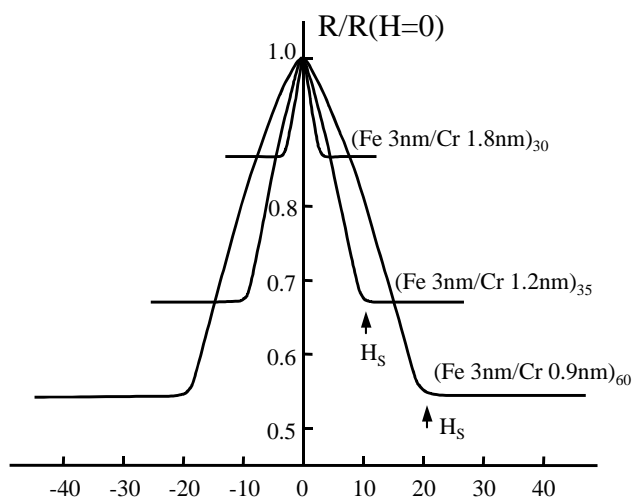

Magnetic field (kG) Fig.6 Normalized resistance versus applied magnetic field for several antiferromagnetically required to overcome the antiferrom. Aretic interlayer coupling between the Fe layers and align their magnetizations parallel. After Baibich et al.

In 1990 a significant step towards the industrial application of GMR was made by Parkin et al. who demonstrated that GMR can be observed in multilayers deposited by sputtering rather than the much slower MBE growth process. They succeeded in obtaining similar GMR values on sputtered polycrystalline $\mathrm{Fe} / \mathrm{Cr}$ multilayers ${ }^{4}$ and later found a sizeable GMR of $120 \%$ on $\mathrm{Co} / \mathrm{Cu}$ multilayers. ${ }^{44}$ In the magnetic multilayers the successive ferromagnetic layers are exchange-coupled through a nonmagnetic spacer layer. Parkin et al. found that the sign of the coupling oscillates between ferromagnetic and antiferromagnetic with increasing thickness of the spacer layer. The magnitude of GMR is also oscillating from a finite value to zero as the spacer thickness increases, as shown in Fig.7 for the $\mathrm{Fe} / \mathrm{Cr}$ multilayer. These oscillations in GMR reflect the oscillations in the interlayer coupling. Sizeable values of GMR are observed when the coupling is antiferromagnetic, since this provides an antiparallel alignment of the magnetizations in the successive ferromagnetic layers at zero magnetic field, as for $d_{C r}=1 \mathrm{~nm}$ and $d_{C r}=2.5 \mathrm{~nm}$ in Fig.7. No GMR (a much diminished GMR in Fig.7) is observed when the coupling is ferromagnetic which prevents the change in the relative alignment of the magnetizations as the applied field is varied, as for $d_{C r}=1.8 \mathrm{~nm}$ in Fig.7. As we saw earlier, antiferromagnetic coupling is not a necessary condition for GMR to occur. All that is necessary is that the magnetic moments of the layers are not locked by the ferromagnetic coupling, but can be reoriented by an applied field.

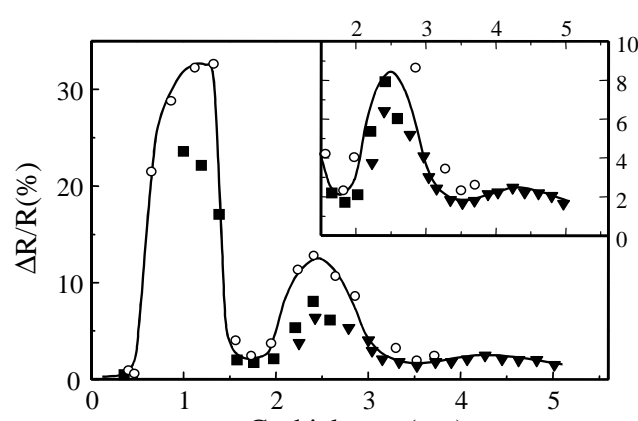

Fig.7 Saturation magnetoresistance at $4.2 \mathrm{~K}$ versus $\mathrm{Cr}$ thickness for $\mathrm{Si}(111) / \mathrm{Cr}(10 \mathrm{~nm}) /$ $[\mathrm{Fe}(2 \mathrm{~nm}) / \mathrm{Cr}(\mathrm{t})] \mathrm{Cr}(5 \mathrm{~nm})$ multibyers deposited at various temperatures: triangles and squares $-40^{\circ} \mathrm{C}(\mathrm{N}=30)$; circles $-125^{\circ} \mathrm{C}(\mathrm{N}=20)$. After Parkin et al . $^{4}$

Although the highest values of GMR were measured in antiferromagnetically-coupled magnetic multilayers, these multilayers are not the best materials for technological applications. This is due to the large magnetic fields, which are required to saturate the magnetization of the multilayers and to obtain a sizeable change in the resistance. For example, as is evident from Fig.6, the saturation field in the $\mathrm{Fe} / \mathrm{Cr}$ multilayers are of the order of $10-20 \mathrm{kG}$ which is three orders of magnitude higher than the fields required for applications. The sensitivity, which is defined as $\Delta R / R$ per unit magnetic field, is much too small. It is of the order of $0.01 \% / \mathrm{G}$, as compared, e.g., to $1 \% / \mathrm{G}$ AMR in permalloy. A search of low field GMR structures in which an antiparallel configuration of the magnetizations could be achieved by different means, as compared to the aniferromagnetic interlayer coupling, resulted in the invention of pseudo spin valves and spin valves.

The pseudo spin valves, shown in Fig.2b, combine hard and soft magnetic layers, which have different coercivities. The magnetic moments of the soft and hard magnetic layers switch at differen values of the applied magnetic field providing a field range in which they are antiparallel and the resistance is higher. ${ }^{5-7}$ In the experiments of Barnas et al. ${ }^{5} \mathrm{Co} / \mathrm{Au} / \mathrm{Co}$ trilayers were used with an $\mathrm{A}$ spacer layers thick enough so that there was no exchange coupling between the Co films. The first $\mathrm{Co}$ 
layer was evaporated on [110]-oriented GaAs, whereas the second one was grown on the Au space layer, which resulted in different coercive fields of the two Co films. Fig.8a shows the hysteresis loop for the $\mathrm{Co} / \mathrm{Au} / \mathrm{Co}$ trilayer obtained at room temperature by the magneto optic Kerr effect (MOKE). I is seen that there is a range of magnetic fields where the magnetizations of the Co layers are aligned antiparallel, as is indicated by the arrows in Fig.8a. Fig.8b shows the resistance trace obtained by scanning through the hysteresis loop. At sufficiently high magnetic fields the magnetizations of both ferromagnetic films are parallel and the resistance is low. The resistance increases, however, each time the antiparallel alignment is achieved during a scan through the hysteresis loop.

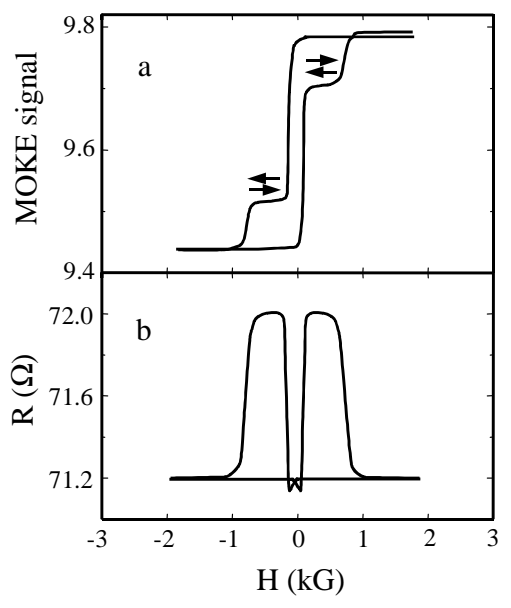

Fig.8 MOKE (a) and resistance (b) hysteresis loops of a Co/Au/Co trilayer with Co thickness of $10 \mathrm{~nm}$ and $\mathrm{Au}$ interlayer thickness of $6 \mathrm{~nm}$ at room temperature. The two Co films have different coercive fields. A range of magnetic fields, in which the magnetizations of both Co layers are aligned antiparallel and the resistance is high, is indicated by arrows. After Barnas et $a l .^{5}$

A structure, which gives a much better performance from the point of view of applications, is the spin valve introduced by Dieny $e t a l .^{8}$ The simplest form of the spin valve structure is shown in Fig.2c. It consists of a magnetically soft ferromagnetic layer (free layer), a non-magnetic metal space layer and a second ferromagnetic layer (pinned layer), which is exchange-coupled to an antiferromagnetic layer. The exchange coupling between the antiferromagnetic layers and the adjacent ferromagnetic layer creates unidirectional exchange anisotropy, i.e. pins the magnetization of this ferromagnetic layer creates unidirectional (exchange anisotropy, i.e. pins the magnetization of this of the pinned ferromagnetic layer is therefore centred about a non-zero bias field, $H_{B}$. On the contrary, the magnetic hysteresis loop of the free layer is centred close to zero field, provided the magnetic coupling between the ferromagnetic layers across the spacer layer is weak enough. The magnetic moments of the two ferromagnetic layers are thus aligned antiparallel in the field range between zero and $H_{B}$.

This behavior is illustrated in Figs.9(a,b) which show, respectively, the magnetization curve and the change in resistance relative to parallel alignment, measured at room temperature, for a sample with the following structure: $\mathrm{Si} / \mathrm{Ni}_{80} \mathrm{Fe}_{20}(15 \mathrm{~nm}) / \mathrm{Cu}(2.6 \mathrm{~nm}) / \mathrm{Ni}_{80} \mathrm{Fe}_{20}(15 \mathrm{~nm}) / \mathrm{FeMn}(10 \mathrm{~nm}) / \mathrm{Ag}(2 \mathrm{~nm}){ }^{8}$ The magnetization curve shows two separate hysteresis loops. The loop with the smaller coercivity corresponds to the reversal of the free $\mathrm{NiFe}$ layer, while the loop shifted by exchange anisotropy to around $H_{B}=90 \mathrm{G}$ corresponds to the reversal of the magnetization of the pinned NiFe layer. Thus, as the field $H$ is swept, the magnetizations of the two NiFe layers change from parallel alignment for $H$ lower than $2 \mathrm{G}$ or higher than $135 \mathrm{G}$ to antiparallel alignment between these two values. It is thus apparent that the change in resistance of Fig. $9 \mathrm{~b}$ is related to the change in relative orientation between the magnetizations of the two ferromagnetic layers. The steep resistance change in the smal field range close to $H=0$ is now used for many low field applications, such as sensors, read heads, and magnetic random access memories.

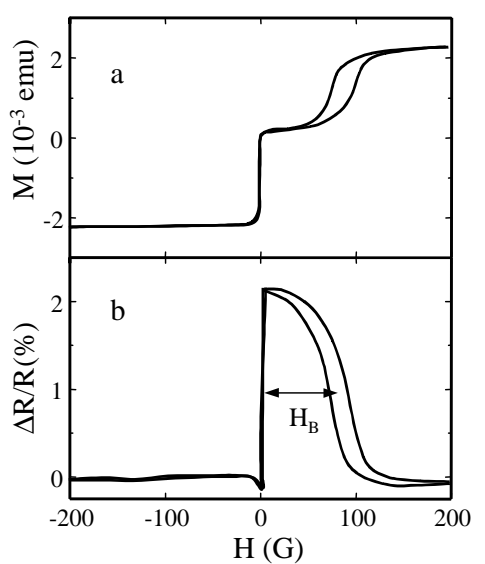

Fig.9 Magnetization curve (a) and relative change in resistance (b) for $\mathrm{Si} / \mathrm{Ni}_{80} \mathrm{Fe}_{20}(15 \mathrm{~nm}) /$ $\mathrm{Cu}(2.6 \mathrm{~nm}) / \mathrm{Ni}_{80} \mathrm{Fe}_{20}(15 \mathrm{~nm}) / \mathrm{Fe}_{50} \mathrm{Mn}_{50}(10 \mathrm{~nm}) / \mathrm{Ag}(2 \mathrm{~nm})$ spin valve. The field is applied parallel to the exchange anisotropy field created by FeMn. $H_{B}$ denotes the exchange-bias field. After Dieny et $a l^{8}$

Magnetic granular materials represent another structure, which displays the GMR effect. ${ }^{9}$ They consist of a non-magnetic metal alloyed with a ferromagnetic metal, which precipitates into granules, as is schematically shown in Fig.2d. The size of the granules depends on the solubility of the ferromagnetic material in the nonmagnetic matrix and on growth and annealing conditions and can be as small as $2 \mathrm{~nm}$. Although the granules can be magnetically coupled, in the absence of the applied field their magnetic moments are randomly-oriented. Applying the magnetic field aligns the moments of the granules, which results in the resistance drop. This behaviour is illustrated in Fig.10, which displays the field dependence of the relative change in the resistance for heterogeneous $\mathrm{Co}_{x} \mathrm{Cu}_{1 \times}$ alloys for two concentrations of $\mathrm{Co}: \mathrm{x}=0.19$ and $\mathrm{x}=0.28 .^{9}$ Although the major part of the samples were found to be disordered fcc alloys, the presence of Co-rich clusters results in a sizeable magnetoresistance at low temperatures. The saturation fields, which are required to align the moments, are as high as in the antiferromagnetically-coupled multilayers, i.e. of the order of $10 \mathrm{kG}$ This fact makes the applicability of granular materials fairly limited. In addition, the magnitude of GMR in granular materials at room temperature is strongly reduced due to superparamagnetic relaxation, which originates from thermal fluctuations of the magnetic moments of the granules. 


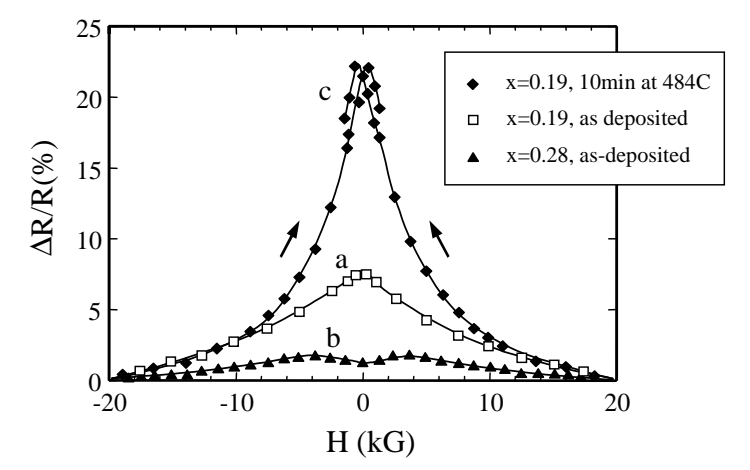

Fig.10 Magnetic field dependence of $\Delta R / \mathrm{R}=[R(H)-R(H=20 \mathrm{kG})] / R(H=20 \mathrm{kG})$ in granular $\mathrm{Co}_{\mathrm{x}} \mathrm{Cu}_{1-\mathrm{x}}$ films. Curves $a$ and $b$ measured at $T=100 \mathrm{~K}$, curve $c$ measured at $T=10 \mathrm{~K}$. After Berkowitz
et ${ }^{9}$

In the following sections we review experimental results on composition, thickness, roughness, impurity, outer boundary, temperature and angular dependence of GMR in magnetic multilayers an spin valves.

\section{Composition dependence}

Since the discovery of GMR a large number of magnetic multilayer structures, which display the GMR effect, have been discovered. It was found that the magnitude of GMR varies considerably depending on the chemical constituents of the multilayer. The highest published values of GMR to date are $220 \%$ in $\mathrm{Fe} / \mathrm{Cr}$ multilayers ${ }^{39}$ and $120 \%$ in $\mathrm{Co} / \mathrm{Cu}$ multilayers. ${ }^{44}$ Sizeable values of GMR were also obtained in the multilayers: $\mathrm{Co} / \mathrm{Ag}-22 \%$ at room temperature (RT), ${ }^{46} \mathrm{Ni} / \mathrm{Ag}-28 \%$ at $4.2 \mathrm{~K},{ }^{47}$ $\mathrm{Ni} / \mathrm{Cu}-9 \%$ at $4.2 \mathrm{~K},{ }^{48} \mathrm{Ni}_{80} \mathrm{Fe}_{20} / \mathrm{Cu}-18 \%$ at $\mathrm{RT}^{49}{ }^{4} \mathrm{Ni}_{80} \mathrm{Fe}_{20} / \mathrm{Ag}-17 \%$ at $\mathrm{RT},{ }^{50}$ and $\mathrm{Ni}_{80} \mathrm{Fe}_{20} / \mathrm{Au}-$ $12 \%$ at RT. ${ }^{51}$ On the other hand, low GMR values of the order of $1 \%$ or less were measured in $\mathrm{Fe} / \mathrm{Mo},{ }^{52} \mathrm{Fe} / \mathrm{Au}{ }^{53} \mathrm{Co} / \mathrm{Cr}^{4}{ }^{4} \mathrm{Co} / \mathrm{Al}^{54},{ }^{54}$ and $\mathrm{Co} / \mathrm{Ir}^{55}$ multilayers. No GMR was found in $\mathrm{Ni}_{80} \mathrm{Fe}_{20} / \mathrm{NM} /$ $\mathrm{Ni}_{80} \mathrm{Fe}_{56} / \mathrm{Fe}_{50} \mathrm{Mn}_{50}$ spin valve structures with $\mathrm{Ta}, \mathrm{Al}, \mathrm{Cr}$ and $\mathrm{Pd}$ as the nonmagnetic (NM) spacer layers. ${ }^{56}$

Why are some of the multilayers highly magnetoresistive, whereas the others are not? All the above multilayers contain ferromagnetic $3 d$ metals, which should have a pronounced spin asymmetry in their conductivity due to the presence of exchange split $d$ bands. It appears that the spin asymmetry in the band structure is a necessary but not sufficient condition for high GMR values. As was noted in section 2, GMR to a great extent is determined by the ferromagnetic metal/nonmagnetic metal pair, rather than by an individual material considered separately. For example, GMR was found to be much lower in $\mathrm{Co} / \mathrm{Cr}$ and $\mathrm{Fe} / \mathrm{Cu}$ multilayers $\left(3 \%\right.$ in $\mathrm{Co} / \mathrm{Cr}^{4}$ and $5.5 \%$ in $\left.\mathrm{Fe} / \mathrm{Cu}^{57}\right)$, as compared to the $\mathrm{Fe} / \mathrm{C}$ and $\mathrm{Co} / \mathrm{Cu}$ multilayers.

There are two factors, which are crucial for obtaining high values of GMR. These are the ban matching and the lattice matching between the ferromagnetic and nonmagnetic metals. As has been already explained in section 2, a good band matching for one spin orientation between ferromagnetic metal (FM) and a non-magnetic metal (NM) implies high transmission for this spin across the FM/NM interface. On the other hand, a large band mismatch for the other spin orientation implies that the transmission of this spin is poor. In addition, roughness and intermixing near the interfaces results in spin-dependent scattering as a consequence of the lateral randomness in the atomic potentials. Large spin dependence in scattering arises if the atomic potentials of the two type of atoms are similar (matched) for one spin orientation but strongly dissimilar (mismatched) for the other spin orientation.

Lattice matching of the subsequent layers is also a very important factor for GMR. Lattice mismatch leads to the formation of misfit dislocations and other structural defects at the interfaces. Scattering by these defects in the nonmagnetic spacer layer is spin-independent resulting in a reduction of GMR. Although the scattering by defects in a ferromagnetic layer could be spindependent, the spin asymmetry in the scattering potentials will vary depending on structural details The presence of various types of defects will make the average of the scattering potential only weakly-dependent on the spin, which can lead to reduced values of GMR.

These two conditions, i.e. band and lattice matching, are almost perfectly satisfied in $\mathrm{Co} / \mathrm{Cu}$ and $\mathrm{Fe} / \mathrm{Cr}$ multilayers. There is an excellent band matching between the majority-spin electrons of $\mathrm{Co}$ and $\mathrm{Cu}$ and the minority-spin electrons of $\mathrm{Fe}$ and $\mathrm{Cr}$. On the other hand, there is a strong band mismatch between the minority spins in $\mathrm{Co}$ and $\mathrm{Cu}$ and the majority spins of $\mathrm{Fe}$ and $\mathrm{Cr}$. The lattice matching is also almost perfect in these systems. Thin films of Co grow in the $f_{c c}$ structure with the lattice parameter of $3.56 \mathrm{~A}$, which is only $2 \%$ less than the lattice parameter of $3.61 \AA \mathrm{A} f c c \mathrm{Cu}$. Both $\mathrm{Fe}$ an in $\mathrm{Cr}$. Thus, it is not surprising that the highest values of GMR are obtained in $\mathrm{Co} / \mathrm{Cu}$ and $\mathrm{Fe} / \mathrm{Cr}$ multilayers.

$\mathrm{Ni}$ and permalloy $\left(\mathrm{Ni}_{80} \mathrm{Fe}_{20}\right)$ have the $f c c$ structure with a lattice parameter close to that in Co and $\mathrm{Cu}$. Like $\mathrm{Co}$, these materials are strong ferromagnets with entirely filled majority-spin $d$ bands, so tha there is good band matching between the majority-spin electrons in $\mathrm{Ni}$ and $\mathrm{Ni}_{80} \mathrm{Fe}_{20}$ on the one han and in $\mathrm{Cu}$ on the other hand. This fact explains the relatively high values of GMR in $\mathrm{Ni} / \mathrm{Cu}$ and $\mathrm{Ni}_{80} \mathrm{Fe}_{20} / \mathrm{Cu}$ multilayers. Nevertheless, the magnitude of GMR in these multilayers is normally les that in $\mathrm{Co} / \mathrm{Cu}$ multilayers (e.g., refs.48,49). This difference can be explained by the stronger disorder in magnetic moments at the $\mathrm{Ni} / \mathrm{Cu}$ and $\mathrm{Ni}_{80} \mathrm{Fe}_{20} / \mathrm{Cu}$ interfaces as compared to the $\mathrm{Co} / \mathrm{Cu}$ interface, as will be discussed in section 8 .

The noble metals $\mathrm{Ag}$ and $\mathrm{Au}$ can serve as good spacer materials in Co-, $\mathrm{Ni}$ - and $\mathrm{Ni}_{80} \mathrm{Fe}_{20}$-based multilayers and spin valves. These metals have electronic and atomic structure similar to $\mathrm{Cu}$, although not as good band and lattice matching with the $3 d$ ferromagnets. For example, $\mathrm{Ni}_{80} \mathrm{Fe}_{20} / \mathrm{Ag}$, and $\mathrm{Ni}_{80} \mathrm{Fe}_{20} / \mathrm{Au}$ permalloy-based multilayers show GMR values of about $20 \%$ at room temperature and reveal a high sensitivity of the resistance to the applied field, $0.2 \% / \mathrm{G}$, and low interlayer coupling. ${ }^{50,5}$ This combination makes them attractive for applications. Unfortunately, the growth of these multilayers represents a real problem. For example the $\mathrm{Ni}_{80} \mathrm{Fe}_{20} / \mathrm{Ag}$ multilayer has to be deposited at liquid-nitrogen temperatures in order to attain the required integrity of the layers.

Other nonmagnetic materials are poor for using as spacer layers in $3 d$-ferromagnet-based multilayers. For example, Al, though a good conductor, displays an unimpressive performance in GMR structures (e.g., ref.54). It produces a strong spin-independent scattering at the interfaces due to the electronic structure mismatch for both spin orientations. This is similar to what one would expect in $\mathrm{Co} / \mathrm{Cr}$ multilayers. Ta is a bad conductor due to a high density of states at the Fermi energy. It is not surprising that GMR is negligible in systems where Ta is used as a spacer layer.

It is interesting that the systems with highest GMR, such as $\mathrm{Fe} / \mathrm{Cr}, \mathrm{Co} / \mathrm{Cu}, \mathrm{Co} / \mathrm{Ag}, \mathrm{Ni}_{80} \mathrm{Fe}_{20} / \mathrm{Au}$ and $\mathrm{Ni}_{80} \mathrm{Fe}_{20} / \mathrm{Ag}$, are all immiscible. This fact indicates that intermixing at the interfaces is not favourable to GMR, and contradicts the expectation that intermixing produces strong spin-dependent scattering potentials. One of the reasons for this might be a reduction in the magnetic moments in the intermixed regions which negatively effects GMR (e.g., ref.58). In addition, the intermixing may result in misaligned spins, which are weakly coupled with the ferromagnetic layer, or magnetically "dead" layers. We come back to this issue in section 7 and 8 . 
A number of attempts have been made to use half-metallic materials in GMR structures. ${ }^{59-61}$ Halfmetallic compounds are characterized by the coexistence of metallic behaviour for one electron spin and insulating behaviour for the other spin. The electronic density of states is, therefore, $100 \%$ spinpolarized at the Fermi level, and the conductivity is dominated by single-spin charge carriers. Calculations predict, for example, that bulk $\mathrm{NiMnSb}^{62}$ and $\mathrm{CrO}_{2}{ }^{63}$ are half-metallic ferromagnets. Ideally incorporation of a $100 \%$ spin-polarized ferromagnet into a GMR multilayer should lead to switching between finite and infinite resistance within the CPP geometry as the magnetezation of alternative ferromagnetic layers switches from parallel to antiparallel, i.e. to an infinitely large GMR Unfortunately, experiments so far show an unimpressive behavior of GMR systems based on half metals. The highest value of GMR of about $7 \%$ is found in CPP measurements on $\mathrm{NiMnSb} / \mathrm{Cu} / \mathrm{NiMnSb}$ trilayers at liquid helium temperature. ${ }^{60}$ This result is far short of the infinite value of CPP GMR expected from a half-metallic-based structure. A possible reason for this is the poor quality of the NiMnSb films, in particular at the $\mathrm{NiMnSb} /$ spacer interfaces, resulting in a reduced spin polarization and/or a significant spin-flip scattering due to misaligned magnetic moments.

\section{Nonmagnetic layer thickness dependence}

When considering the dependence of GMR on the non-magnetic layer thickness in magnetic multilayers and spin valves one should compare the resistances of the perfectly parallel and antiparallel magnetic configurations. The presence of the interlayer exchange coupling leads to oscillations in GMR, similar to those displayed in Fig.7. This oscillatory contribution to GMR reflect the extent of antiparallel alignment, which is achieved at zero magnetic field, rather than an intrinsic variation in GMR. Spin valves are in this sense better for studying the spacer thickness dependence of GMR than magnetic multilayers. This is due to the pinned ferromagnetic layer, which keeps the direction of its magnetization and helps to maintain an antiparallel alignment of the magnetizations in a certain field interval, provided that the ferromagnetic interlayer coupling is not stronger than the exchange-bias field. However, at small spacer thicknesses the magnetic layers may become strongly coupled ferromagnetically due to the presence of pinholes in the nonmagnetic film, leading to a decreased GMR ratio.

The dependence of GMR on the non-magnetic layer thickness in spin valves was studied by Dieny $e t$ al. ${ }^{64}$ Fig. 11 shows the variation of GMR as a function of the thickness of the non-magnetic layer (NM) in spin valve structures with composition: $\mathrm{Si} / \mathrm{Co}(7 \mathrm{~nm}) / \mathrm{NM}\left(d_{N M}\right) / \mathrm{Ni}_{80} \mathrm{Fe}_{20}(5 \mathrm{~nm}) /$ $\mathrm{Fe}_{50} \mathrm{Mn}_{50} \mathrm{Mn}(8 \mathrm{~nm})$ with $\mathrm{NM}=\mathrm{Cu}$ and $\mathrm{Au}$. As is seen from the figure, the value of GMR decrease monotonically with increasing non-magnetic layer thickness. This decrease can be qualitatively ascribed to two factors. (i) With increasing spacer thickness the probability of scattering increases as the conduction electrons traverse the spacer layer, which reduces the flow of electrons between the ferromagnetic layers and consequently reduces GMR. (ii) The increasing thickness of the nonmagnetic layer enhances the shunting current within the spacer, which also reduces GMR. These two contributions to GMR can be phenomenologically described by the following expression: ${ }^{21}$

$$
\frac{\Delta R}{R}=\left(\frac{\Delta R}{R}\right)_{0} \frac{\exp \left(-d_{N M} / l_{N M}\right)}{\left(1+d_{N M} / d_{0}\right)}
$$

The exponential factor represents the probability that an electron is not scattered within the NM layer. The factor in the denominator describes the shunting effect due to the NM layer. The parameter $l_{N M}$ is related to the mean free path of the conduction electrons in the spacer layer. One expects that $l_{N M}$ will be less than the mean free path in the spacer layer $\lambda_{N M}$, due to the fact that electrons which most effectively contribute to GMR have out-of-plane velocities. Dieny et $a l .^{21}$ proposed that for systems of practical interest $l_{N M}$ is approximately equal to half of the mean free path $\lambda_{N M}$. The parameter $d_{0}$ is an effective thickness, which depends on the conductance of the system in the absence of the NM layer $(\Delta R / R)_{0}$ is a normalization coefficient.

Although formula (5.1) is a purely phenomenological expression, it contains a significant part of the physics involved. As we will see in section 12, within a Boltzmann approach to free electrons the simple exponential in expression (5.1) is replaced by more complicated exponential integrals over various incidence angles of the conduction electrons with respect to the plane of the layers. Nevertheless, the typical variation of GMR versus non-magnetic layer thickness remains qualitatively the same.

It was found that the $\mathrm{Cu}$ and $\mathrm{Au}$ thickness dependence of GMR, illustrated in Fig.11, can be fitted well by using decay lengths of $l_{C u}=6 \mathrm{~nm}$ and $l_{A u}=5 \mathrm{~nm}$ respectively. ${ }^{21}$ These decay lengths are determined by scattering in the spacer, due to phonons, grain boundaries, and other defects, and are correlated with the mean free path $\lambda_{N M}$. The smaller value found for Au is consistent with the highe resistivity of $\mathrm{Au}$, deduced from measurements on sputtered samples: $\lambda_{A u}=8.5 \mathrm{~nm}(\rho=7 \mu \Omega \mathrm{cm})$ for $\mathrm{Au}$ versus $\lambda_{C u}=11.5 \mathrm{~nm}(\rho=5 \mu \Omega \mathrm{cm})$ for $\mathrm{Cu}$.

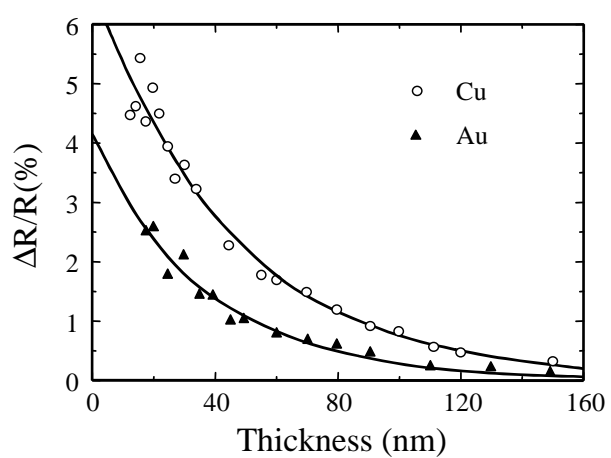

Fig.11 Magnetoresistance at room temperature versus thickness of the noble-metal layer in spin valves $\mathrm{Si} / \mathrm{Co}(70 \mathrm{~nm}) / \mathrm{NM}\left(d_{N M}\right) / \mathrm{Ni}_{8} \mathrm{Fe}_{20}(5 \mathrm{~nm}) / \mathrm{Fe}_{50} \mathrm{Mn}_{50}(8 \mathrm{~nm}) / \mathrm{NM}(1.5 \mathrm{~nm})$ with $\mathrm{NM}=\mathrm{Cu}$ and Au. The solid lines represent fits according to Eq.(3.1). After Dieny et al. ${ }^{6}$

As is evident from Fig.11, the values of GMR are higher for the $\mathrm{Cu}$ spacer layer than for the $\mathrm{Au}$ spacer layer. Indeed, extrapolation to zero interlayer thickness gives $\Delta R / R$ of $6.4 \%$ for Cu versus $4.1 \%$ for $\mathrm{Au}$. This fact was ascribed to a lower transmission through the ferromagnetic/noble-metal interfaces for $\mathrm{Au}$ than for $\mathrm{Cu}$, which reduces the intensity of the flow of electrons that continuously escape from each ferromagnetic layer across the interfaces. ${ }^{64}$ The low GMR values for Au may also reflect the higher spin-orbit scattering expected of the heavier element, which leads to spin-flip scattering in the spacer layer. The effect of the microstructure may also be important: the large lattice mismatch between ferromagnetic layers and Au may result in misfit dislocations and be an additiona cause of the lower GMR.

GMR in magnetic multilayers versus thickness of the non-magnetic spacer layer behaves in a similar fashion as in spin valves. Figs.12a,b display values of GMR in $\mathrm{Co} / \mathrm{Cu}$ multilayers measured a relatively large $\mathrm{Cu}$ thicknesses, so that the interlayer exchange coupling is small. ${ }^{65}$ Note that the interlayer exchange coupling decreases with increasing $\mathrm{Cu}$ thickness much faster than GMR, such that the exchange coupling fields become much weaker than the saturation fields. Therefore, GMR in 
Figs.12 results from the random arrangement of magnetic domains in successive magnetic layers Parkin et al. ${ }^{65}$ found that at $\mathrm{T}=4.2 \mathrm{~K}$ GMR decays approximately as $1 / d_{C u}$ (Fig.12a), which is consistent with Eq. (5.1) provided that the decay length $l_{N M}$ is large. As was explained above, this behavior is the direct consequence of the shunting of the electric current due to increasing thicknes of the spacer layers. At room temperature the scattering within the spacer layers diminishes the flow of electrons from one magnetic layer to neighboring magnetic layers and therefore reduces the magnitude of GMR. Such scattering is related to volume scattering within the interior of the spacer layers due to electron-phonon interactions. The value of GMR then decays as in Eq. (5.1) with $l_{N M}$ $=32 \mathrm{~nm}$ and is shown in Fig. $12 \mathrm{~b}$ by the solid line.

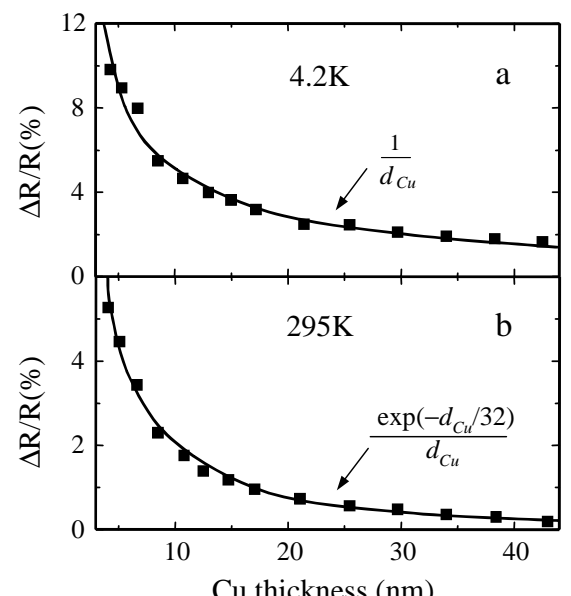

Fig.12 Saturation magnetoresistance versus $\mathrm{Cu}$ spacer layer thickness for several series of multilayers of the form, $\mathrm{Si}(111) / \mathrm{Ru}(5 \mathrm{~nm}) /\left[\mathrm{Co}(1.1 \mathrm{~nm}) / \mathrm{Cu}\left(d_{\mathrm{Cu}}\right)\right]_{6} / \mathrm{Ru}(1.5 \mathrm{~nm})$. Data are shown for temperatures of $4.2 \mathrm{~K}$ (a) and $295 \mathrm{~K}$ (b). The actual curves shown in the figure have the form of $\Delta R / R=0.28+55.4 /\left(1.3+d_{\mathrm{Cu}}\right)$ and $\Delta R / R=28.9 /\left(0.43+d_{\mathrm{Cu}}\right) \exp \left(-d_{\mathrm{Cu}} / 31.8\right)$ at 4.2 and $295 \mathrm{~K}$ respectively. $d_{\mathrm{Cu}}$ is given in $\mathrm{nm}$. After Parkin $e t$ al. ${ }^{6}$

As we see from the experimental results and will see from the theoretical analysis within the semiclassical free-electron models (section 12), the mean free path appears to be the scaling length for the thickness dependence of GMR within the CIP geometry. GMR decays monotonically as a function of the spacer layer thickness. The highest values of GMR can be achieved when the spacer layer is as thin as possible and therefore has only a small amount of bulk scattering. The reduction of the spacer layer thickness is however limited by pinholes through the nonmagnetic material, which prevent the antiparallel alignment of the magnetizations and therefore suppress the magnetoresistance.

\section{Magnetic layer thickness dependence}

A typical variation of the magnitude of GMR versus the thickness of the free ferromagnetic layer in the $\mathrm{FM}\left(d_{F M}\right) / \mathrm{Cu}(2.2 \mathrm{~nm}) / \mathrm{Ni}_{80} \mathrm{Fe}_{20}(5 \mathrm{~nm}) / \mathrm{Fe}_{50} \mathrm{Mn}_{50}(8 \mathrm{~nm}) / \mathrm{Cu}(1.5 \mathrm{~nm})$ spin valve versus the thicknes of the ferromagnetic free layer $\mathrm{FM}=\mathrm{Co}, \mathrm{Ni}_{80} \mathrm{Fe}_{20}$ and $\mathrm{Ni}$ is plotted in Fig.13. ${ }^{66}$ As is evident from the figure, the three curves have very similar shapes characterized by a broad maximum between 6 and $10 \mathrm{~nm}$. As was argued by Dieny, ${ }^{21}$ the position of the maximum depends on the location of the spin-dependent scattering centers. In the case of interfacial spin-dependent scattering, the maximum is located at smaller thicknesses than for bulk spin-dependent scattering. The appearance of the maximum is explained by the following arguments. ${ }^{64}$ The decrease in GMR at large magnetic laye thickness is due to the increasing shunting of the current in the inner part of the ferromagnetic layers. The decrease in GMR at low thickness is due to the scattering at the outer boundaries (substrate, buffer layer or capping layer). This scattering significantly affects GMR when the thickness of the ferromagnetic layer becomes smaller than the longer of the two mean-free paths associated with the up- and down-spin electrons (see also section 9).

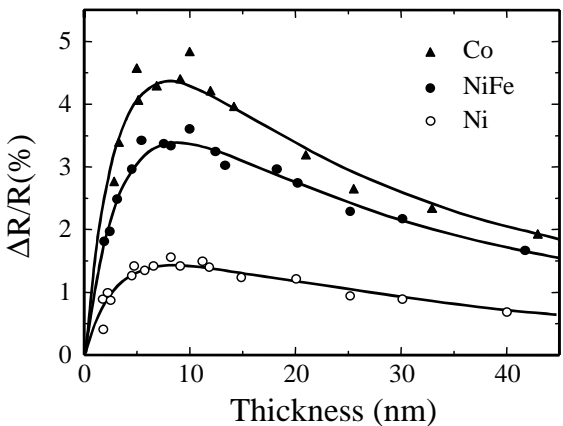

Fig.13 Magnetoresistance in $\mathrm{FM}\left(d_{F M}\right) / \mathrm{Cu}(2.2 \mathrm{~nm}) / \mathrm{Ni}_{80} \mathrm{Fe}_{20}(5 \mathrm{~nm}) / \mathrm{Fe}_{50} \mathrm{Mn}_{50}(8 \mathrm{~nm}) / \mathrm{Cu}(1.5 \mathrm{~nm})$ spin $\mathrm{i}_{80} \mathrm{Fe}_{20}$ and $\mathrm{Ni}$ at room .

Phenomenologically, the variation of spin-valve MR with the thickness of the ferromagnetic lay ers can be fairly well represented by the following expression ${ }^{66}$ (see Fig.13):

$$
\frac{\Delta R}{R}=\left(\frac{\Delta R}{R}\right)_{0} \frac{1-\exp \left(-d_{F M} / l_{F M}\right)}{\left(1+d_{F M} / d_{0}\right)}
$$

The numerator describes the variation of the scattering rates of the electrons with thickness $d_{F M}$. I characterizes the angle-averaged probability for an electron with the longest mean free path to be scattered within the ferromagnetic layer before being scattered diffusively at the outer boundary of the spin valve. This factor is responsible for the decrease of GMR at low thicknesses $d_{F M}$ : if the ferromagnetic layers are too thin the contrast between the spin-dependent mean free paths decreases due to the stronger diffuse scattering of the electrons with the longer mean free path at the outer boundaries. $l_{F M}$ is therefore related to the longest mean free path in the ferromagnetic layer $\lambda_{F M}$. As argued by Dieny, ${ }^{8}$ it is expected that $l_{F M} \approx 1 / 2 \lambda_{F M}$. The denominator describes the shunting of the current within the ferromagnetic layers, so that $d_{0}$ is an effective thickness which represents the shunting of the current in the rest of the structure, i.e. in all layers except the ferromagnetic layer whose thickness is varied. $(\Delta R / R)_{0}$ is a normalization coefficient. Although a more accurate expression describes GMR within the Boltzmann approach (see section 12), formula (6.1) contains a significant part of the physics involved. 
In magnetic multilayers with a large number of repetitions, a maximum in the value of GMR is normally observed when the thickness of the magnetic layers is varied from a monolayer to a few $\mathrm{nm}$, i.e. less than in spin valves. For example, Sato et al.$^{67}$ have found that the optimal thickness of the permalloy layers in $\left(\mathrm{Cu} / \mathrm{Ni}_{80} \mathrm{Fe}_{20}\right)_{60}$ multilayers is typically $1-3 \mathrm{~nm}$. The main difference between the spin valves and multilayers is the reduced effect of the outer boundary scattering for the former. Although the decrease of GMR at high FM layer thickness can still be explained by the shunting current within the FM layers, the decrease of magnetoresistance at small thicknesses has a differen origin and can be explained as follows. ${ }^{21}$ In the case of bulk spin-dependent scattering, the decrease of GMR at low $d_{F M}$ is due to insufficient scattering of the electrons with short mean free paths, which reduces the spin-asymmetry in the conductivity. The critical thickness below which the electrons with short mean free paths are insufficiently scattered is the mean free path of the electrons in the ferromagnetic layers, i.e. of the order of $1-2 \mathrm{~nm}$ in permalloy ${ }^{68}$ In the case of interfacial spin-dependent scattering, this critical thickness of the ferromagnetic material is the minimum thickness required to establish the electronic properties of the FM/NM interface.

\section{Roughness dependence}

As was known from earliest experiments on Fe/Cr multilayers, GMR is very sensitive to the growth conditions and the structure of the interfaces. It is expected that interface roughness will enhance the magnetoresistance due to an increase in spin-dependent scattering. A number of experiments aiming to correlate the interface roughness and GMR in magnetic multilayers have been performed. Indeed, a few experiments have demonstrated that the value of GMR in $\mathrm{Fe} / \mathrm{Cr}$ multilayers has a tendency to increase with roughness. For example, Fullerton et al. ${ }^{69}$ fabricated sputtered $\mathrm{Fe} / \mathrm{Cr}$ multilayers with variable roughness by changing the sputtering gas pressure and varying the sputtering power. The structure of the samples was thoroughly characterized by high and low-angle X-ray diffraction. They found that GMR is higher when the intensity of the low-angle diffraction peak is smaller, which implies rougher interfaces. From the magnetization measurements they showed that the enhancement in GMR is not due to the improvement in antiferromagntic alignment and concluded that spin-dependent scattering at the interfaces is enhanced by roughness.

More recently Schad et al..$^{70}$ fabricated a series of high-quality epitaxial $\mathrm{Fe} / \mathrm{Cr}(001)$ multilayers characterized by a negligible number of bulk defects, so that the dominant contribution to scattering resulted from interface roughness. The interface roughness was varied through annealing at different temperatures and was quantitatively analyzed by specular and diffuse synchrotron X-ray diffraction technique. Schad et al. found that the magnitude of GMR increases with decreasing the lateral correlation length of roughness, $\xi_{x}$. This can be seen from Fig.14a, in which the triangles show the saturation resistivity, $\rho_{S}$, the change in the resistivity, $\Delta \rho$, and the GMR ratio, $\Delta \rho / \rho_{S}$, for the samples with constant roughness amplitude $\eta$, and variable lateral correlation length $\xi_{\mathrm{x}}$. A further increase in the annealing temperature leads to increasing roughness height, $\eta$, resulting in a further enhancemen of GMR, as can be seen from the circles in Fig.14a.

This enhancement of GMR with interfacial roughness observed in monocrystalline $\mathrm{Fe} / \mathrm{C}$ multilayers is in contrast to what was observed earlier on polycrystalline $\mathrm{Fe} / \mathrm{Cr}$ superlattices in experiments by the same group. ${ }^{71}$ A reduction of GMR was found with increasing the amplitude of the interface roughness having a constant correlation length, as is shown in Fig.14b. This fact demonstrates that spin-dependent scattering is very sensitive to the details of the microstructure of the interfaces. For example, polycrystalline samples could provide efficient diffusion channels along the grain boundaries, so that annealing can facilitate creating interdiffused interfaces, which according to ref 39 reduces GMR. In addition, steps at the interfaces of the polycrystalline samples often appear a the grain boundas, resulting the grain boundaries, resulting in a variable spin asymmetry in the scattering potential due to different structural and compositional environment at the steps. On average, the scattering potential associated with the roughness can become spin-independent resulting in a reduced GMR.

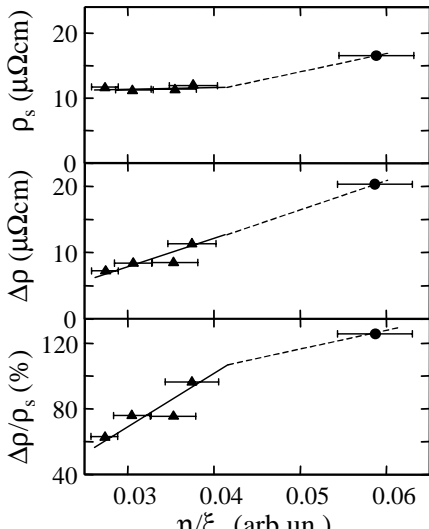

$\eta / \xi_{\mathrm{x}}$ (arb.un.)

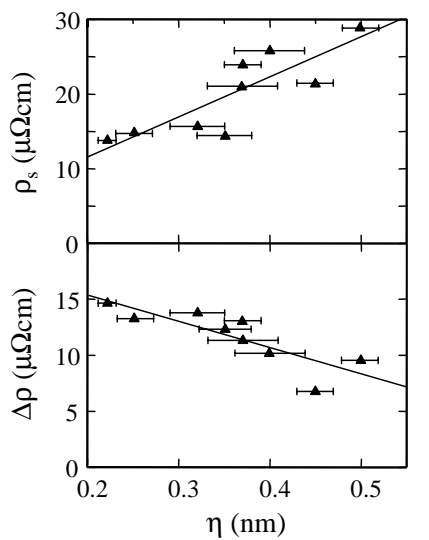

Fig.14 Transport properties of (001)-oriented monocrystalline (a) and polycrystalline (b) $\mathrm{Fe} / \mathrm{Cr}$ multilayers. Variations in the antiferromagnetic coupling are taken into account by dividing $\Delta \rho$ and $\Delta \rho / \rho_{S}$ by $\left(1-M_{R} / M_{S}\right)$, where $M_{R}$ and $M_{S}$ are the remanent and saturation $\Delta \rho$ annetizations respectively. (a) The triangular data points correspond to the samples with the constant roughness amplitude $\eta$, so that only the lateral correlation length $\xi$ was varying. (b) The lateral correlation length of the roughness is constant $\xi_{x} \approx 9 \mathrm{~nm}$. After Schad
et al. ${ }^{70,71}$

From the experiments on $\mathrm{Fe} / \mathrm{Cr}$ multilayers one can conclude that increasing the density of step and the roughness height at compositionally-sharp monocrystalline regions of the interfaces enhances GMR in these multilayers. On the other hand, interface roughness associated with interdiffused regions and a high density of defects, such as grain boundaries, is likely to reduce GMR.

Opposite to these $\mathrm{Fe} / \mathrm{Cr}$ multilayers, no enhancement of GMR has been observed in $\mathrm{Co} / \mathrm{Cu}$ multilayers with increasing interface roughness. For example, Kano et al. ${ }^{72}$ varied the sharpness of the interfaces in sputtered $\mathrm{Co} / \mathrm{Cu}$ multilayers by changing the substrate temperature. The degree of roughness was determined by the X-ray satellite peak intensity. They found that the GMR ratio decreases as the substrate temperature increases and concluded that roughness reduces GMR. Suzuk and $\mathrm{Taga}^{73}$ succeeded in preparing $\mathrm{Co} / \mathrm{Cu}$ superlattices with well-controlled interfacial roughness by magnetron sputtering. The interfaces between $\mathrm{Co}$ and $\mathrm{Cu}$ were modified by codeposition, so that the thickness of the intermixed $\mathrm{CoCu}$ layer was varied from 0 to $0.25 \mathrm{~nm}$. In these samples, only the interfacial region was modified, while the morphology and the crystallinity of the multilayer remain unchanged. They found that interfacial roughness mainly contributes to the residual resistivity and the spin dependence of the scattering at the interfaces is weak. They also concluded that the GMR ratio decreases with increasing interfacial roughness.

The suppression of GMR in $\mathrm{Co} / \mathrm{Cu}$ multilayers with increasing interfacial roughness is probably due to a significant change in the magnetic state of the Co atoms in the intermixed regions. The magnetic moments of these atoms might be reduced and misaligned with the magnetization of the Co layer. The spin asymmetry of scattering by these atoms is obviously strongly reduced, resulting in a decrease of GMR with increasing roughness. $\mathrm{Fe} / \mathrm{Cr}$ multilayers are electronically more stable with respect to roughness, so that the electronic state of the atoms at the stepped interfaces is similar to that in the bulk of the layers. This fact is supported by first-principle calculations which show that the 
atomic moments at the rough $\mathrm{Fe} / \mathrm{Cr}$ interfaces do not depend significantly on the nearest-neighbour environment. $^{58}$

\section{Impurity dependence}

Since the magnitude of GMR is related to the asymmetry in the scattering rates within the two conduction channels, it was expected that modifying the spin-dependent scattering by introducin appropriate impurities either at the interfaces or in the bulk of the ferromagnetic layers would enhance GMR. Scattering asymmetries have been indirectly determined from measurements of the resistivity of magnetic ternary alloys. ${ }^{31,32}$ A number of attempts have, therefore, been made to find a correlation between the magnitude of the scattering asymmetries in bulk magnetic alloys and the magnitude of GMR in magnetic multilayers.

Gurney et $_{\text {al. }}{ }^{74}$ inserted thin layers $(\leq 0.4 \mathrm{~nm})$ of various impurities, such as Au, Ag, V, Mn, Al, $\mathrm{Ge}$, and Ir at the interfaces between $\mathrm{Fe}$ and $\mathrm{Cr}$ layers in $\mathrm{Fe} / \mathrm{Cr}$ multilayers. They found that inserting $\mathrm{Au}, \mathrm{Ag}, \mathrm{Al}, \mathrm{Ge}$, or Ir strongly reduces GMR, whereas, inserting $\mathrm{V}$ and $\mathrm{Mn}$ does not effect GMR much, as compared to an inserted $\mathrm{Cr}$ layer of same thickness. Johnson and Camley interpreted the results of these experiments in terms of different spin-dependent scattering asymmetries of these impurities in bulk iron. ${ }^{75}$ They found that the impurities, such as $\mathrm{Mn}$ and $\mathrm{V}$, which have a spindependent scattering asymmetry similar to that of $\mathrm{Cr}$ in $\mathrm{Fe}$, do not change GMR substantially. On the other hand the impurities, such as $\mathrm{Al}$ and Ir, with a spin-dependent scattering asymmetry opposite to that of $\mathrm{Cr}$ in $\mathrm{Fe}$, lead to a rapid degradation of the GMR. We note, however, that according to Marrows and Hickey, ${ }^{76}$ inserting the impurities at the interface of antiferromagnetically-coupled multilayers could easily destroy the interlayer coupling, resulting in a reduction of GMR due to imperfect antiferromagnetic alignment.

Parkin ${ }^{77}$ demonstrated that inserting a very thin layer of $\mathrm{Co}$ at the $\mathrm{Ni}_{80} \mathrm{Fe}_{20} / \mathrm{Cu}$ interfaces results in a dramatic increase in GMR. Fig.15a shows the room temperature resistance response to the applied magnetic field in a $\mathrm{Si} / \mathrm{Ni}_{80} \mathrm{Fe}_{20}(5.3 \mathrm{~nm}) / \mathrm{Cu}(3.2 \mathrm{~nm}) / \mathrm{Ni}_{80} \mathrm{Fe}_{20}(2.2 \mathrm{~nm}) / \mathrm{Fe}_{50} \mathrm{Mn}_{50}(9 \mathrm{~nm}) / \mathrm{Cu}(1 \mathrm{~nm})$ spin valve and in the same spin valve with $0.25 \mathrm{~nm}$ thick Co layers added at each $\mathrm{Ni}_{80} \mathrm{Fe}_{20} / \mathrm{Cu}$ interface. A is seen from this figure, the value of GMR increases by a factor of two, demonstrating the strong effect of the inserted Co layer. A careful analysis of GMR as a function of the Co layer thickness shows that the magnetoresistance can be enhanced from $2.9 \%$ up to $6.4 \%$, the Co layer thickness scale of the enhancement being just $0.23 \mathrm{~nm}$ (see Fig.15b). The positive effect of the Co layers on GMR in the permalloy-based spin valves was found to be strongly localized at the interfaces. By varying the distance of the $0.5 \mathrm{~nm}$ Co layer from the interface, $d$, no significant increase in GMR was found for $d>0.5 \mathrm{~nm}$ (see Fig. $15 \mathrm{~b}$ ). Contrary to inserting Co layers at the interfaces of permalloy-based spin valves, adding a permalloy layer at the $\mathrm{Co} / \mathrm{Cu}$ interfaces in $\mathrm{Si} / \mathrm{Co}(5.7 \mathrm{~nm}) / \mathrm{Cu}(2.4 \mathrm{~nm}) / \mathrm{Co}(2.9 \mathrm{~nm}) /$ $\mathrm{Fe}_{50} \mathrm{Mn}_{50}(10 \mathrm{~nm}) / \mathrm{Cu}(1 \mathrm{~nm})$ spin valves reduces the value of GMR from $6.8 \%$ to $3.9 \%$ with the $\mathrm{Ni}_{80} \mathrm{Fe}_{20}$ thickness scale of just 2.8nm (see Fig.15d).

The results of these experiments are explained by the dominant contribution to GMR from spindependent scattering at the interfaces. Theoretically Inoue et $a l^{78}$ argued that the minority-spin scattering at $\mathrm{Co} / \mathrm{Cu}$ interfaces is larger than at $\mathrm{Ni}_{80} \mathrm{Fe}_{20} / \mathrm{Cu}$ interfaces, due to the larger mismatch in the minority-spin $d$ atomic energy levels for the former. Experimentally, on the other hand, the effect appears to arise from the stabilising role of $\mathrm{Co}$ on the magnetic moments at the interfaces. In general, appears to arise from the stabilising role of Co on the magnetic moments at the interfaces. In general,
the magnetic moments at interfaces (especially rough interfaces) can be very different from those in the magnetic moments at interfaces (especially rough interfaces) can be very different from those in
the bulk. Speriosu et al.$^{79}$ found that at room temperature there is a substantial reduction in the magnetization of permalloy near the interfaces with $\mathrm{Cu}$, which is equivalent to a magnetically-dead layer of $0.2 \mathrm{~nm}$ thickness. On the contrary, a much thinner dead-layer of only $0.1 \mathrm{~nm}$ was found for $\mathrm{Co} / \mathrm{Cu}$ multilayer. The non-magnetic layers at the interfaces are detrimental to GMR. These layers are a source of strong spin-independent scattering. Misoriented spins also reduce GMR due to spin mixing and spin-flip scattering. The presence of reduced Ni moments and non-collinear Fe moments on interdiffused $\mathrm{Ni}_{80} \mathrm{Fe}_{20} / \mathrm{Cu}$ interfaces is supported by first-principle calculations. ${ }^{80}$ Placing a small amount of $\mathrm{Co}$ at the interface dramatically increase the collinearity of magnetic moments and stabilise the magnetic moments of $\mathrm{Ni}$ at the $\mathrm{Ni}_{80} \mathrm{Fe}_{20} / \mathrm{Cu}$ interface, thereby enhancing GMR.
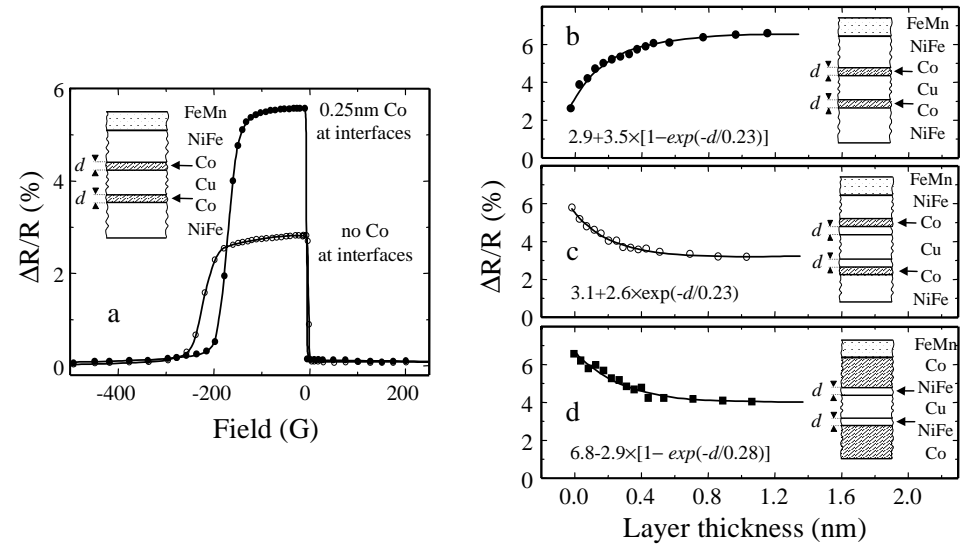

Fig.15 Effect of a thin layer inserted at the interfaces in spin valves. (a) Resistance versus magnetic field for $\mathrm{Si} / \mathrm{NiFe}(5.3) / \mathrm{Cu}(3.2) / \mathrm{NiFe}(2.2) / \mathrm{FeMn}(9) / \mathrm{Cu}(1)$ spin valve without (open circles) and with (filled circles) $0.25 \mathrm{~nm}$ thick Co layers added at each $\mathrm{NiFe} / \mathrm{Cu}$ interface. Dependence of the saturation magnetoresistance on (b) Co interface layer thickness, $d_{C o}$, in $\mathrm{Si} / \mathrm{NiFe}\left(5.3-d_{C o}\right) / \mathrm{Co}\left(d_{C o}\right) / \mathrm{Cu}(3.2) / \mathrm{Co}\left(d_{C o}\right) / \mathrm{NiFe}\left(2.2-d_{C o}\right) / \mathrm{FeMn}(9) / \mathrm{Cu}(1)$ spin valves, (c) distance $d$ of a $0.5 \mathrm{~nm}$ thick Co layer from the $\mathrm{NiFe} / \mathrm{Cu}$ interfaces in $\mathrm{Si} / \mathrm{NiFe}(4.9-$ d) $/ \mathrm{Co}(5) / \mathrm{NiFe}(d) / \mathrm{Cu}(3) / \mathrm{NiFe}(d) / \mathrm{Co}(5) / \mathrm{NiFe}(1.8-d) / \mathrm{FeMn}(9) / \mathrm{Cu}(1)$ spin valves, and (d) $\mathrm{NiFe}$ interface layer thickness, $d_{\text {NiFe }}$, in $\mathrm{Si} / \mathrm{Co}\left(5.7-d_{\text {NiFe }}\right) / \mathrm{NiFe}\left(d_{\text {NiFe }}\right) / \mathrm{Cu}(2.4) / \mathrm{FeNi}\left(d_{\text {NiFe }}\right) /$ $\mathrm{Co}\left(2.9-d_{\text {NiFe }}\right) / \mathrm{FeMn}(10) / \mathrm{Cu}(1)$ spin valves. Note that NiFe stands for permalloy and the layer thicknesses are given in $n \mathrm{~m}$. Experiments are performed at room temperature. After
Parkin. ${ }^{77}$

A number of interesting examples of impurity effects have been obtained within the CPP geometry. As was explained in Sec.3 using a simple series resistor model, the GMR can be inverted (i.e. acquires an opposite sign) if the spin asymmetries in scattering are opposite in consecutive ferromagnetic layers. Vouille et al. ${ }^{81}$ have found that $\mathrm{Ni}_{95} \mathrm{Cr}_{5} / \mathrm{Cu} / \mathrm{Co} / \mathrm{Cu}$ multilayers, in which $\mathrm{N}$ layers are doped by $5 \%$ of $\mathrm{Cr}$ impurities, display the inverse GMR when $\mathrm{Ni}_{95} \mathrm{Cr}_{5}$ thickness is more than $2 \mathrm{~nm}$. According to Campbell and $\mathrm{Fert}^{32} \mathrm{Cr}$ impurities in Ni scatter more strongly the majorityspin electrons, which makes the bulk scattering spin asymmetry of the NiCr layers less than unity, i.e. $\alpha_{\mathrm{NiCr}}<1$. This is opposite to the Co layers, which are characterized by $\alpha_{\mathrm{Co}}>1$, resulting in the inverse GMR. The inverse GMR was also found in other multilayers of the type $\mathrm{FM} / \mathrm{Cu} / \mathrm{Co} / \mathrm{Cu}$, where $\mathrm{FM}=\mathrm{Ni}_{1-\mathrm{x}} \mathrm{Cr}_{\mathrm{x}}, \mathrm{Co}_{1-\mathrm{x}} \mathrm{Cr}_{\mathrm{x}}, \mathrm{Co}_{1-\mathrm{x}} \mathrm{Fe}_{\mathrm{x}}$ and $\mathrm{Fe}_{1-\mathrm{x}} \mathrm{V}_{\mathrm{x}}{ }^{81,82}$ The comparison of the bulk scattering spin asymmetries $\alpha_{\mathrm{FM}}$ extracted from these experiments with the previous results obtained for bulk alloys ${ }^{31,32}$ shows that the sign of $\alpha_{\mathrm{FM}}$ is the same but the magnitude is generally much smaller. The inversion of GMR in the experiments of Hsu et al. ${ }^{82}$ and Vouille et al. ${ }^{81}$ appears to occur at thicknesses of the FM layer above a certain critical thickness, so that there was a crossover in the sign of GMR at this thickness. This was ascribed to the competition between bulk scattering in the FM, 
which has spin asymmetry $\alpha_{\text {bulk }}<1$, and interface scattering at the $\mathrm{FM} / \mathrm{Cu}$ interface, which was assumed to have spin asymmetry $\alpha_{\text {int }}>1$. We note, however, that at low FM thickness the interface resistance might become dependent on the layer thickness, which would make the interpretation of these experiments more complicated (see section VI).
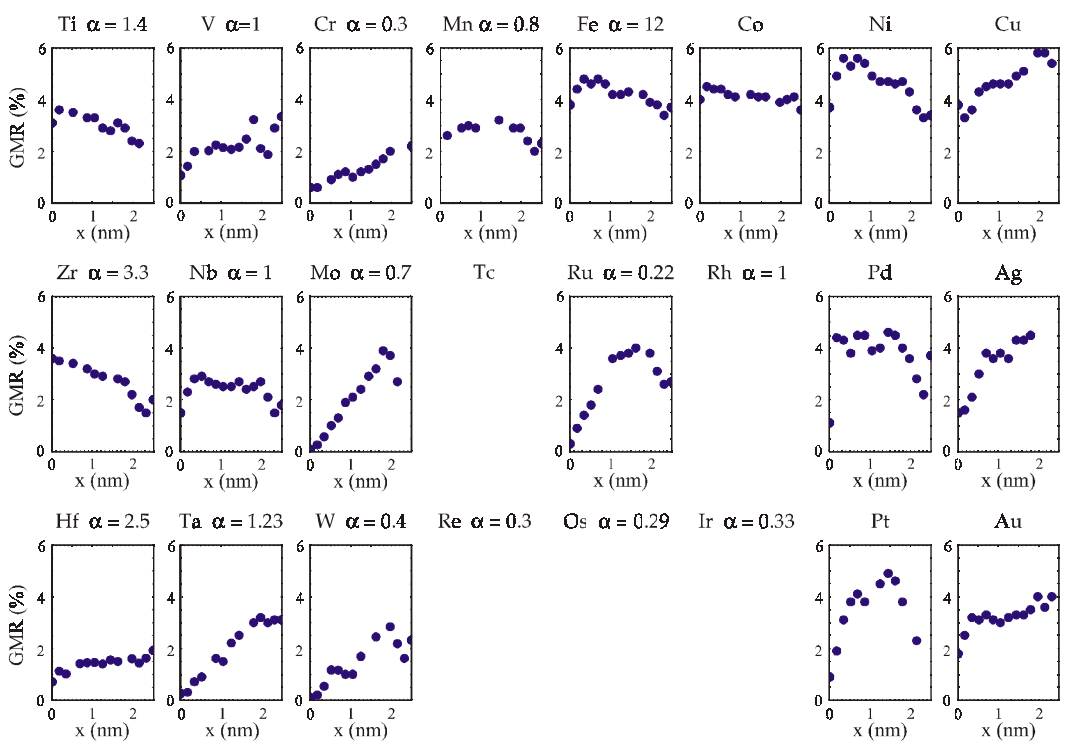
Fig.16 Position dependence of the giant magnetoresistance for various transition metal impurities in the Co layer in Si/Ta (5n)/Co(2.5n $-x) / \delta / \mathrm{Co}(x) / \mathrm{Cu}(3 \mathrm{~nm}) / \mathrm{Co}(x) / \mathrm{Co}(2.5 \mathrm{~nm}-x) / \mathrm{FeMn}(8 \mathrm{~nm}) /$ values not at the top of increases in each oraph the $\delta$ laner. The f the Co layers. The graph for Co con be regrded as the control experiment. After Mace and Hickey. ${ }^{76}$

Very interesting experiments have been performed recently by Marrows and Hickey ${ }^{76}$ who inserted a very thin (sub-monolayer) $\delta$-layer of various elements in a $\mathrm{Co} / \mathrm{Cu} / \mathrm{Co}$ spin valve at variou distances from the interfaces. Some of their results are shown in Fig.16, in which the GMR ratio is plotted against the position of the dopant $\delta$-layer for a variety of elements from the central part of the transition metal series. As is evident from the figure, the value of GMR can be reduced or enhanced depending on the nature of the impurity and its location within the spin valve. For example, ferromagnetic $3 d$ impurities $\mathrm{Fe}$ and $\mathrm{Ni}$ enhance GMR when they are close to but just behind the $\mathrm{Co} / \mathrm{Cu}$ interface. For materials with $\alpha<1$, i.e. $\mathrm{Cr}, \mathrm{Mo}, \mathrm{Ta}$, and $\mathrm{Ru}$, the magnetoresistance is totally suppressed when they are placed at the interface, but it is recovered as they move back into the bulk of the Co layer. Materials to the right of Co, that might be expected to have $\alpha>1$, namely Pd, Pt, and the noble metals, can suppress the GMR somewhat at the interface, but it is very rapidly recovered as they move into the layer. It was also found that $4 f$ magnetic impurities are altogether damaging to the GMR. A quantitative interpretation of these experiments is a challenge for first-principle modeling.

\section{Outer boundary dependence}

As we saw above, the value of GMR is higher in magnetic multilayers than in spin valves. The latter consist of just two ferromagnetic layers separated by a nonmagnetic spacer layer. With increasing number of FM/NM bilayers within a multilayer the value of GMR monotonically increase until it reaches saturation. Fig.17a shows an example of such a variation of GMR, obtained by Plaskett and $\mathrm{McGuire}^{83}$ in experiments on $\mathrm{Cu}(1 \mathrm{~nm}) /[\mathrm{Co}(1 \mathrm{~nm}) / \mathrm{Cu}(1 \mathrm{~nm})]_{\mathrm{N}}$ multilayers. They found that at $\mathrm{T}=4.2 \mathrm{~K}$ GMR grows up to $35 \%$ with increasing $\mathrm{N}$ up to 128 , clearly displaying a tendency to saturate (see Fig.17a). The lower values of GMR in these experiments as compared to those in ref.38 are explained by a weak antiferromagnetic coupling. One of the factors, which may play a role in increasing GMR with the number of bilayers, is an improvement in the structural quality for the thicker multilayers. However, the major factor, which is responsible for the behavior of GMR versus $\mathrm{N}$ shown in Fig.17a, is the presence of diffuse scattering at the outer boundaries of the multilayer Indeed, if the longest mean free path is much larger that the total thickness of the magnetic multilayer diffuse outer-boundary scattering reduces the conductivity of the "good" conduction channel and hence effects negatively GMR. Plaskett and McGuire used the Fuchs-Sondheimer expression for thin film resistivity ${ }^{84,85}$ in order to estimate the mean free paths, as is shown in Fig.17b. They found mean free path of $47 \mathrm{~nm}$ for the saturated state of the $\mathrm{Co} / \mathrm{Cu}$ multilayer, which although possibly an overestimate gives the right order of magnitude. We see, therefore, that outer boundary scattering is very important characteristic of spin valves because their thickness is comparable to (or even less than) the mean free path.
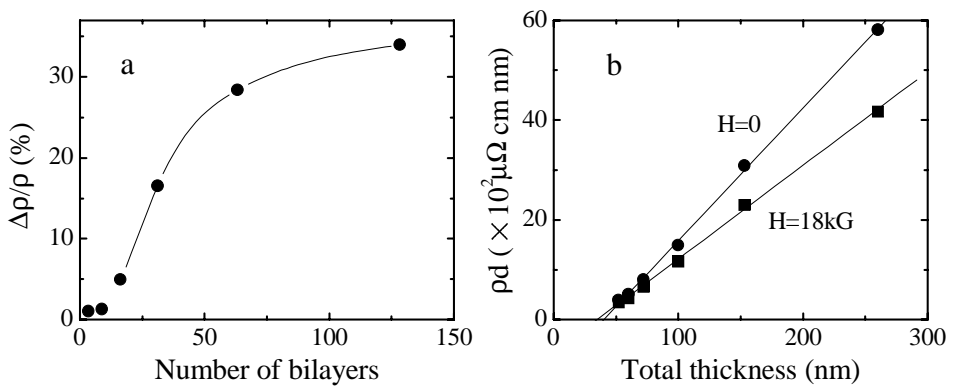

Fig.17 GMR as a function of the number of bilayers $N$ (a) and resistivity as a function of the GMR as a function of the number of bilayers $N$ (a) and resistivity as a function of the
multilayer thickness $d$ (b) in $\mathrm{Cu}(1 \mathrm{~nm}) /\left[\mathrm{Co}(1 \mathrm{~nm} / \mathrm{Cu}(1 \mathrm{~nm})]_{\mathrm{N}}\right.$ multilayers, measured at
$\mathrm{T}=4.2 \mathrm{~K}$. GMR is determined from the resistivity at $H=0$ and $H=18 \mathrm{kG}$ except for $N=128$, for which $H=25 \mathrm{kG}$. Solid lines in (b) represent the Fuchs-Sondheimer approximation for thick film resistivity, ${ }^{2}, \rho d=\rho_{0}(d+3 \lambda / 8)$, where $\rho_{0 \text { is }}$ is the bulk resistivity and $\lambda$ is the mean free path. After Plaskett and McGuire. ${ }^{83}$

The outer boundary scattering in spin valves occurs at the interfaces between the pinned layer and the pinning layer and between the sense layer and the cap layer, which is used for protecting the structure from oxidation or corrosion. Antiferromagnetic $\mathrm{Fe}_{50} \mathrm{Mn}_{50}^{8}$ and ferrimagnetic $\mathrm{Tb}_{23} \mathrm{Co}_{77}{ }^{86}$ are frequently used as pinning layers. The scattering at $\mathrm{FM} / \mathrm{FeMn}$ and $\mathrm{FM} / \mathrm{TbCo}$ interfaces is commonly regarded as entirely diffuse due to the highly disordered atomic and magnetic structures of the antiferromagnetic layers and the interfaces. In addition, although these materials are highly resistive (resistivity is about $100 \mu \Omega \mathrm{cm}$ at room temperature), they still contribute to the shunting curren 
within the spin valve structure, thereby reducing GMR. The same effect occurs at the FM/Ta interface when Ta is used as the protective cap layer.

GMR in spin valves can be improved if antiferromagnetic $\mathrm{NiO}$ is used as a pinning layer Anthony et al.$^{87}$ fabricated bottom spin valves of the type $\mathrm{NiO} / \mathrm{NiFe} / \mathrm{Co} / \mathrm{Cu} / \mathrm{Co} / \mathrm{NiFe}$ and obtained GMR ratio of $13 \%$ at room temperature (thin Co layers at the interfaces were used to enhance $\mathrm{GMR}^{77}$ ). An even higher value of GMR, namely $15 \%$, was obtained by Swagten $e^{2} \mathrm{al}^{88}$ in a spin valve of the type $\mathrm{NiO} / \mathrm{Co} / \mathrm{Cu} / \mathrm{Co} / \mathrm{Cu} / \mathrm{NiO}$, in which the top $\mathrm{NiO}$ layer served as a protective layer and the top $\mathrm{Cu}$ layer magnetically insulated the free layer from exchange biasing. These relatively high values of GMR are the result of the fact that $\mathrm{NiO}$ is an insulator and consequently does not contribute to the shunting current. Moreover, due to the relatively large band gap of $\mathrm{NiO}$ (about $4 \mathrm{eV}$ ) it impedes penetration of electrons into the bulk and therefore provides a mechanism for specular scattering at the interface between the pinned and pinning layers. Contrary to diffuse scattering, specular scattering reflects electrons back through the spin valve allowing them to propagate across the spacer layer many times, thereby increasing spin filtering effects. The degree of specular scattering at $\mathrm{FM} / \mathrm{NiO}$ interfaces is related to the interface quality because disorder can easily make the scattering diffuse.

A certain degree of specular scattering was argued to be the origin of high values of GMR in the experiments performed by Egelhoff et al..$^{89,90}$ on NiO-based symmetric spin valves. A symmetric spin valve $^{87}$ contains one free ferromagnetic layer sandwiched between two pinned magnetic layers, separated by nonmagnetic spacer layers, which allows higher magnitudes of GMR to be obtained than in ordinary spin valves. The symmetric spin valve, which was studied by Egelhoff et al., had the structure of $\mathrm{NiO} / \mathrm{Co} / \mathrm{Cu} / \mathrm{Co} / \mathrm{Cu} / \mathrm{Co} / \mathrm{NiO}$ and displayed $\mathrm{GMR}$ values exceeding $20 \%$ at room temperature. Specular scattering of electrons at $\mathrm{Co} / \mathrm{CoO}$ interfaces was also suggested to be the reason for the enhancement of GMR by up to $17 \%$ at room temperature, obtained in bottom spin valves of the type $\mathrm{NiO} / \mathrm{Co} / \mathrm{Cu} / \mathrm{Co}$, in which the top Co layer was slightly oxidized. ${ }^{91,90}$ Sugita et al..$^{92}$ regarded the high magnitude of GMR of 28\% obtained in an epitaxially-grown symmetric spin valve with an $\alpha$ $\mathrm{Fe}_{2} \mathrm{O}_{3}$ pinning layer, i.e. $\alpha-\mathrm{Fe}_{2} \mathrm{O}_{3} / \mathrm{Co} / \mathrm{Cu} / \mathrm{Co} / \mathrm{Cu} / \mathrm{Co} / \alpha-\mathrm{Fe}_{2} \mathrm{O}_{3}$, as an indication of specular electron reflection at the $\mathrm{Co} / \alpha-\mathrm{Fe}_{2} \mathrm{O}_{3}$ interfaces.

The importance of overlayers deposited at the top outer boundary of the $\mathrm{NiO} / \mathrm{Co} / \mathrm{Cu} / \mathrm{Co}$ spin valve was demonstrated by Egelhoff et al ${ }^{93}$ They found that the deposition of about 2 monolayers (ML) of $\mathrm{Au}, \mathrm{Ag}$, or $\mathrm{Cu}$ increases GMR, whereas the deposition of $2 \mathrm{ML}$ of $\mathrm{Ta}, \mathrm{Si}, \mathrm{C}$, or $\mathrm{Ni}_{80} \mathrm{Fe}_{20}$ decreases GMR. These results were interpreted by Egelhoff $e t$ al. as evidence of enhanced specular scattering for the case of $\mathrm{Au}, \mathrm{Ag}$, and $\mathrm{Cu}$, but suppressed specular scattering for the case of $\mathrm{Ta}, \mathrm{Si}, \mathrm{C}$ and $\mathrm{Ni}_{80} \mathrm{Fe}_{20}$ at the interface with Co. However, recent studies have demonstrated that the enhancement of GMR occurs for any top Co layer thickness. ${ }^{94}$ This fact contradicts the plausible explanation in terms of specular scattering, because the specular scattering should reduce GMR if the Co thickness is much larger than the optimum thickness (see section 12). For such Co thicknesses the specularly reflected electrons are not able to cross the spacer layer and contribute instead to the shunting current. Other effects seem to be important and require further investigations.

\section{Temperature dependence}

Many experiments have found that GMR decreases with increasing temperature. Typically, the magnitude of GMR is a factor of two or three smaller at room temperature than at liquid helium temperature. For example, GMR drops by a factor of 3.1 in $\mathrm{Fe} / \mathrm{Cr}$ multilayers ${ }^{95}$ and by a factor of 1.8 in $\mathrm{Co} / \mathrm{Cu}$ multilayers ${ }^{38}$ in this temperature interval.

The major factor, which contributes to the temperature variation of GMR, is inelastic scattering by phonons. Although electron-phonon scattering conserves spin, it enhances the saturation resistivity of the multilayer, effecting negatively the GMR ratio. In addition, it shortens the mean free path in the spacer layer, which prevents the flow of electrons between successive ferromagnetic layers and hence spacer layer, which prevents the flow of electrons between successive ferromagnetic layers and hence
reduces GMR. Scattering by phonons in ferromagnetic metals is spin-dependent due to the spin- dependent density of states at the Fermi energy (see sec.3). If other spin-dependent scattering processes are important (e.g., spin-dependent scattering at the interfaces due to interface roughness), which are characterized by a different spin asymmetry, the contribution from electron-phonon scattering necessarily changes GMR. Another non-trivial mechanism reducing GMR is related to interband transitions that are driven by the applied electric field in the presence of spin-independent scattering potentials. This will be discussed in section 17 .
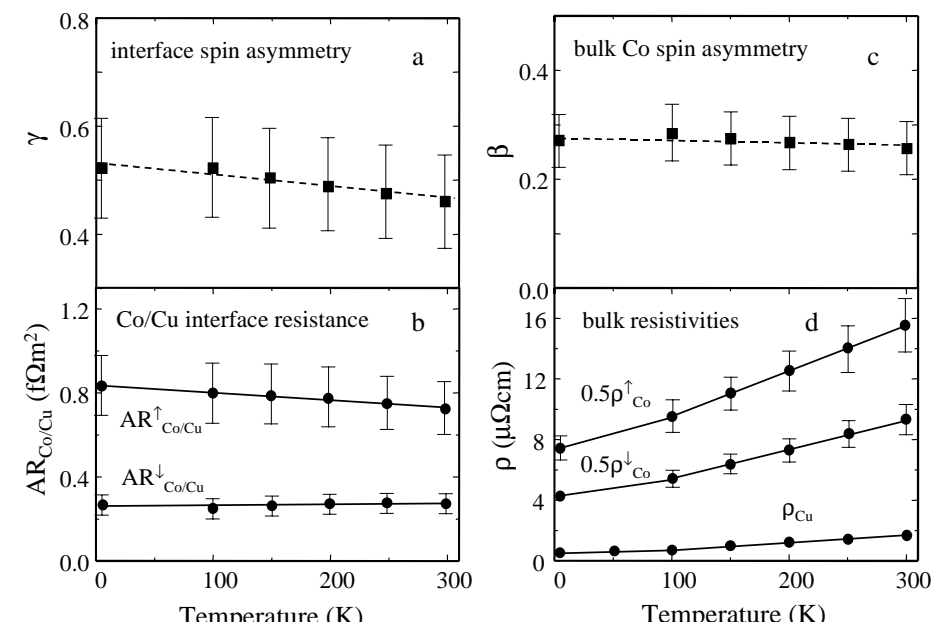

Fig.18 The temperature dependence of the spin-dependent parameters for $\mathrm{Co} / \mathrm{Cu}$ multilayers, a determined from the experiments on grooved substrates using the two-current series resistor model: (a) the spin asymmetry parameter, $\gamma$, of the $\mathrm{Co} / \mathrm{Cu}$ interface, (b) the resistances of the $\mathrm{Co} / \mathrm{Cu}$ interface, (c) the spin asymmetry parameter of the bulk $\mathrm{Co}, \beta,($ d) the resistivity of $\mathrm{Co}$ and Cu layers. $\gamma$ is defined as $\gamma=\left(\alpha_{\mathrm{Co} / / \mathrm{u}^{-1}}-1\right) /\left(\alpha_{\mathrm{Co} / \mathrm{Cu}}+1\right)$, where $\alpha_{\mathrm{Co} / \mathrm{Cu}}$ is the ratio of the interface

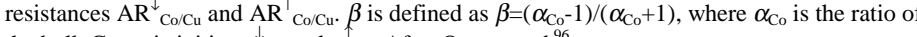
the bulk Co resistivities $\rho_{\text {Co }}^{\downarrow}$ and $\rho_{\text {Co }}$. After Oepts et al. ${ }^{96}$

Another factor, which might influence the temperature variation of GMR, is electron-magnon scattering. Contrary to phonons, scattering by magnons is associated with spin-flip processes which intermix the majority- and minority-spin current channels. At high temperatures when spin fluctuations become sizeable the electron-magnon scattering would inevitably suppress GMR However, whether this effect is important at room temperature and below for GMR in magnetic multilayers based on $3 d$ ferromagnets, which are characterized by very high Curie temperatures, still remains unclear. Dieny et al.$^{66}$ have pointed out that the presence of roughness and interdiffusion a the interfaces weakens the magnetic interactions due to the decreased magnetic moments and the reduced number of magnetic nearest neighbours. These "loose" spins may be more strongly affected by temperature-dependent spin-flip processes than the spins in the bulk of the layers. Dieny et al. have established a correlation between the Curie temperature of the ferromagnetic metal and the slope of the decrease in GMR in spin valves. They found that the thermal variation of GMR in spin valves is weaker for those ferromagnets, which have higher Curie temperature. They argued that this 
correlation is a consequence of spin-flip scattering mainly at the interfaces due to the reduced Curie temperature of the interdiffused interfacial regions.

Very interesting experiments on the temperature dependence of GMR with a quantitative interpretation of the results were reported by Oepts et al. ${ }^{96}$ They measured the temperature variation of GMR in $\mathrm{Co} / \mathrm{Cu}$ multilayers deposited on grooved substrates, as is shown in Fig.3a, so that the geometry in these experiments was similar to CPP GMR. The results were analysed in terms of spindependent bulk and interface resistances within the two-current series resistor model and are shown in dependent bulk and interface resistances within the two-current series resistor model and are shown in
Fig.18. They found that the spin-dependent interface resistances are weakly temperature-dependent (Figs.18a,b), which implies that up to room temperature the major contribution to the interface resistances comes from elastic scattering. On the other hand, as can be seen from Fig.18d, the bulk resistivities of $\mathrm{Co}$ and $\mathrm{Cu}$ increase by more than a factor of two between $4.2 \mathrm{~K}$ and $300 \mathrm{~K}$, reflecting the sizeable contribution from inelastic scattering. However, it was found that the spin asymmetry of the resistivity in the Co layers, $\alpha_{\mathrm{Co}}$, is almost independent of temperature (Fig.18c). The results of these experiments demonstrate, firstly, that phonons, rather than magnons, influence the spindependent resistance of the $\mathrm{Co} / \mathrm{Cu}$ multilayer. Scattering by magnons would inevitably reduce the spin asymmetry of the bulk and interface resistances with increasing temperature due to spin-flip processes. Secondly, the spin asymmetry is about the same for elastic and inelastic scattering in the Co layers. This is evidence that the scattering spin asymmetry is mainly determined by intrinsic properties of bulk $\mathrm{Co}$, namely by its band structure.

\section{Angular dependence}

We have so far considered GMR that arises from parallel and antiparallel magnetizations of the successive ferromagnetic layers. In this section we discuss the variation of the magnetoresistance as function of the angle between the magnetizations $\theta=\theta_{1}-\theta_{2}$. In spin valves which comprise a free and a pinned magnetic layer a continuous change of the angle $\theta$ can be obtained by applying a rotating field, which rotates the magnetization of the free layer, but keeps the direction of the magnetization of the pinned layer fixed. Such an experiment was performed by Dieny et al. ${ }^{8}$ It was found that there are two components contributing to the magnetoresistance: the anisotropic magnetoresistance (AMR), which varies as the cosine squared of the angle between the rotating magnetization and the sensing current, and giant magnetoresistance. By subtracting the contribution from AMR, Dieny et al. found that GMR varies linearly with $\cos \theta$, and can be phenomenologically described by the formula

$$
R(\theta)=R_{P}+\left(R_{A P}-R_{P}\right)(1-\cos \theta) / 2,
$$

where $R_{P}$ and $R_{A P}$ are the resistances of the spin valve for the parallel and antiparallel magnetizations respectively. Such a linear variation of the resistance with $\cos \theta$ was also observed in $\mathrm{Fe} / \mathrm{Cr}$ multilayers. ${ }^{97}$

A theoretical consideration of the angular dependence of GMR within a quantum-mechanical approach and a free-electron model predicts that for a constant potential within the multilayer the conductance, a ther than the resistance, should vary linearly with $\cos \theta^{98,99}$ The same behaviour is a predicted within the semiclassical free-electron the semiclassical freeexperimental study of GMR in $\mathrm{Co} / \mathrm{Ag} / \mathrm{NiFe} / \mathrm{Ag}$ multilayers [100] and is shown in Fig.19. Although the difference between the two descriptions is second order in the GMR ratio, it may be sizeable if the value of GMR is relatively high, which is the case for the $\mathrm{Co} / \mathrm{Ag} / \mathrm{NiFe} / \mathrm{Ag}$ multilayers studied in ref.100. This is evident from Fig.19, which shows almost a perfect linear variation of the conductance with $\cos \theta$, but displays slight non-linearity in the resistance dependence. Although the free-electron theory predicts significant departures from linearity when potential steps at the interfaces are present, ${ }^{98}$ no experiments to date have provided any evidence for such departures. First-principle calculations ${ }^{101}$ of the angular variation of GMR suggest that the functional dependence is essentially of the form $(1-\cos \theta)$, which agrees with experiments.

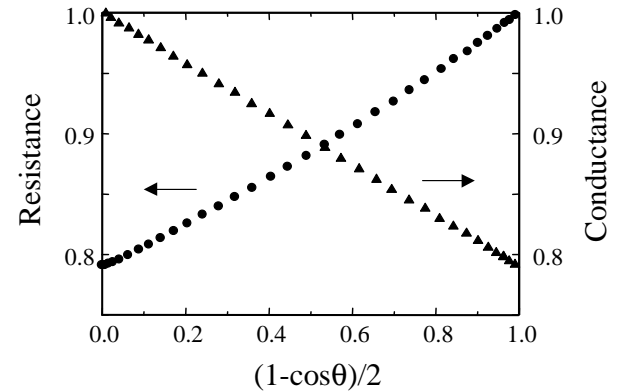

Fig.19 Normalized resistance and conductance versus the cosine of the relative angle between the magnetizations of the soft permalloy layers and hard layers composed of Co clusters for $[\mathrm{Co}(0.4 \mathrm{~nm}) / \mathrm{Ag}(4 \mathrm{~nm}) / \mathrm{NiFe}(4 \mathrm{~nm}) / \mathrm{Ag}(4 \mathrm{~nm})]_{15}$ mutliayer. After Steren et al ${ }^{100}$

The angular variation of GMR has been also studied within the CPP geometry. ${ }^{102}$ Although the deviations from linear dependence of the conductance versus $\cos \theta$ were found to be more pronounced than in the CIP geometry, they still remain relatively small.

\section{FREE-ELECTRON AND SIMPLE TIGHT-BINDING MODELS}

A large number of various theoretical models have been developed to describe GMR. These models differ mainly in the way that they treat the electronic structure and the electronic transport The electronic structure can be described either within a simple free-electron approximation or within an accurate multiband approach. The main advantage of the free-electron theories is that they are physically more transparent and, though simple, can still capture some important physics of GMR This is also the case for simple tight-binding models, which approximate the electronic structure by a single tight-binding band. Multiband models are, however, essential for a quantitative description of GMR. Within these models the electronic structure is described either using parameterized tightbinding bands or first-principle calculations within the local density approximation. The electronic transport can be considered either within semiclassical Boltzmann theory or within quantummechanical theory. The Boltzmann theory of transport is a versatile formalism, which has been widely used for treating GMR. It breaks down, however, in magnetic multilayers of practical interest because the subband energy splitting is comparable with the life-time broadening due to scattering. In these cases quantum-mechanical theory within a multiband treatment of the electronic structure is the bes way to describe GMR. We begin our review of theoretical models for GMR by discussing the semiclassical Boltzmann theory within the free-electron model.

\section{Semiclassical theory}

The resistor model, which has been introduced in section 3 , is too simple to describe correctly CIP GMR in magnetic multilayers and spin valves. This model is based on the assumption that the mean free path is long for both spin channels as compared to layer thicknesses. This approximation is not justified for real layered systems because the mean free path within one of the spin channels is comparable to or even less than the layer thickness. In addition, the resistor model is unable to predic the asymptotic behavior of GMR for large layer thicknesses. 
A more sophisticated quantitative insight into spin-dependent transport can be obtained using the semiclassical Boltzmann theory of transport (e.g., refs.30,103). This theory considers electron transport using classical dynamics, which makes it different from the quantum-mechanical linear response theory of transport that will be considered in section 13. Nevertheless, the semiclassica theory includes many aspects of quantum mechanics. For example, within this approach quantummechanical statistics is used and scattering can be calculated quantum-mechanically assuming a realistic band structure.

Boltzmann theory is based on a semiclassical description of the electrons in metals in the presence of external fields using a statistical distribution function. The distribution function $f(\mathbf{r}, \mathbf{k}, t)$ is defined as the number of electrons with given position $\mathbf{r}$ and wave-vector $\mathbf{k}$ at time $t$. We assume that the two spin states of the electrons are uncoupled and, therefore, the distribution function can be considered independently for the up- and down-spin channels. The Boltzmann transport equation is obtained by balancing the change in the distribution function caused by the applied electric field and the scattering processes that act to bring it back towards equilibrium, i.e.

$$
\frac{d f(\mathbf{r}, \mathbf{k}, t)}{d t}=-\dot{\mathbf{r}} \cdot \nabla_{\mathbf{r}} f-\dot{\mathbf{k}} \cdot \nabla_{\mathbf{k}} f+\left(\frac{\partial f}{\partial t}\right)_{\text {scatt }} .
$$

The first term in this equation describes the electron drift due to their velocity, the second term reflects the acceleration of the electrons due to the applied field and the scattering term describes scattering of the electrons by imperfections in the lattice, such as defects or impurities. It can be written in terms of the probability $P_{\mathbf{k} \mathbf{k}^{\prime}}$ for an electron to scatter between momentum $\mathbf{k}$ and $\mathbf{k}^{\prime}$ :

$$
\left(\frac{\partial f}{\partial t}\right)_{\text {scatt }}=\sum_{\mathbf{k}^{\prime}}\left\{P_{\mathbf{k} \mathbf{k}^{\prime}}[1-f(\mathbf{r}, \mathbf{k}, t)] f\left(\mathbf{r}, \mathbf{k}^{\prime}, t\right)-P_{\mathbf{k} \mathbf{k}}\left[1-f\left(\mathbf{r}, \mathbf{k}^{\prime}, t\right)\right] f(\mathbf{r}, \mathbf{k}, t)\right\},
$$

where the right-hand term describes "scattering-out" processes, in which an electron from an occupie state of momentum $\mathbf{k}$ scatters into unoccupied states $\mathbf{k}^{\prime}$, and the left-hand term describes "scatteringin" processes, in which electrons from occupied states of momentum $\mathbf{k}^{\prime}$ scatter into an unoccupied state $\mathbf{k}$. We are interested in a steady state solution, when the distribution function is no longer changing so that $d f / d t=0$ in equation (12.1). In this case, taking into account the principle of microscopic reversibility, i.e. $P_{\mathbf{k k}^{\prime}}=P_{\mathbf{k} \mathbf{k}}$, and assuming a uniform applied electric field $\mathcal{E}$, we obtain

$$
\mathbf{v}(\mathbf{k}) \cdot \nabla_{\mathbf{r}} f(\mathbf{r}, \mathbf{k})-\frac{e}{\hbar} \boldsymbol{E} \cdot \nabla_{\mathbf{k}} f(\mathbf{r}, \mathbf{k})=\sum_{\mathbf{k}^{\prime}} P_{\mathbf{k} \mathbf{k}^{\prime}}\left[f\left(\mathbf{r}, \mathbf{k}^{\prime}\right)-f(\mathbf{r}, \mathbf{k})\right],
$$

where $e$ is the absolute value of the electron charge and $\mathbf{v}$ is the electron velocity. Aiming at a linear response theory, it is convenient to represent the distribution function as $f(\mathbf{r}, \mathbf{k})=f_{0}(\mathbf{k})+g(\mathbf{r}, \mathbf{k})$, where $g(\mathbf{r}, \mathbf{k})$ is the deviation of the distribution function $f(\mathbf{r}, \mathbf{k})$ from the equilibrium Fermi-Dirac distribution $f_{0}(\mathbf{k})=\left\{1+\exp \left[\left(E(\mathbf{k})-E_{F}\right) / k T\right]\right\}^{-1}$ due to the applied electric field. Substituting this form into Eq. (12.3) and retaining only the lowest order contribution with respect to $\mathcal{E}$ we obtain

$$
\mathbf{v}(\mathbf{k}) \cdot \nabla_{\mathbf{r}} g(\mathbf{r}, \mathbf{k})-e \mathcal{E} \cdot \mathbf{v}(\mathbf{k}) \frac{\partial f_{0}(\mathbf{k})}{\partial E(\mathbf{k})}=\sum_{\mathbf{k}^{\prime}} P_{\mathbf{k} \mathbf{k}^{\prime}}\left[g\left(\mathbf{r}, \mathbf{k}^{\prime}\right)-g(\mathbf{r}, \mathbf{k})\right] .
$$

This is a general representation of the linearized Boltzmann kinetic equation for the description of the electric current, the density of which is given by

$$
\mathbf{j}(\mathbf{r})=-\frac{e}{\Omega} \sum_{\mathbf{k}} \mathbf{v}(\mathbf{k}) g(\mathbf{r}, \mathbf{k}),
$$

where $\Omega$ is the volume of the system. However, the evaluation of Eq.(12.4) is not easy to perform because of the scattering-in term $\sum_{\mathbf{k}^{\prime}} P_{\mathbf{k}^{\prime}} g\left(\mathbf{r}, \mathbf{k}^{\prime}\right)$, which links the values of the distribution function at various momenta. The Boltzmann equation can be considerably simplified using the relaxation time approximation. Within the relaxation time approximation the scattering-in term is neglected which results in

$$
\mathbf{v}(\mathbf{k}) \cdot \nabla_{\mathbf{r}} g(\mathbf{r}, \mathbf{k})-e \mathcal{E} \cdot \mathbf{v}(\mathbf{k}) \frac{\partial f_{0}(\mathbf{k})}{\partial E(\mathbf{k})}=-\frac{g(\mathbf{r}, \mathbf{k})}{\tau(\mathbf{k})},
$$

where $\tau(\mathbf{k})$ is the relaxation time for an electron to scatter out of momentum state $\mathbf{k}$, which is defined

$$
\tau^{-1}(\mathbf{k})=\sum_{\mathbf{k}^{\prime}} P_{\mathbf{k k}^{\prime}} .
$$

In general, neglecting the scattering-in term is not a trivial approximation and has to be justified (e.g. ref.30).

For a bulk homogeneous system it is straightforward within the relaxation time approximation to derive the expression for the conductivity tensor $\sigma^{\mu v}$ which is defined by

$$
j^{\mu}=\sum_{v} \sigma^{\mu v} \mathcal{E}^{v},
$$

where the indices $\mu$ and $v$ denote the Cartesian components. In this case $\nabla_{\mathbf{r}} g(\mathbf{r}, \mathbf{k})=0$ and it follows from Eq. (12.6) that

$$
g(\mathbf{k})=e \tau(\mathbf{k}) \frac{\partial f_{0}(\mathbf{k})}{\partial E(\mathbf{k})} \mathbf{v}(\mathbf{k}) \cdot \mathcal{E} .
$$

Taking the zero-temperature limit, i.e. $\partial f_{n}^{0}(\mathbf{k}) / \partial E_{n}(\mathbf{k})=-\delta\left[E_{n}(\mathbf{k})-E_{F}\right]$, and substituting Eq.(12.9) into Eq.(12.5), we obtain the well-known expression for the conductivity per single spin channel within the relaxation time approximation: ${ }^{30}$

$$
\sigma^{\mu v}=\frac{e^{2}}{\Omega} \sum_{\mathbf{k}} \mathrm{v}^{\mu}(\mathbf{k}) \mathrm{v}^{v}(\mathbf{k}) \tau(\mathbf{k}) \delta\left[E(\mathbf{k})-E_{F}\right] .
$$

In the case of films and multilayers which are assumed to be homogeneous in the $x y$ plane of the layers but inhomogeneous in the $z$ direction perpendicular to the planes (due to the interfaces and boundaries), the distribution function $\mathrm{g}(z, \mathbf{v})$ is dependent on $z$, but independent of $x$ and $y$. In this case the solution of the Boltzmann equation (12.6) takes the form

$$
g^{ \pm}(z, \mathbf{k})=e \tau(\mathbf{k}) \mathcal{E} \cdot \mathbf{v}(\mathbf{k}) \frac{\partial f_{0}(\mathbf{k})}{\partial E(\mathbf{k})}\left[1+A^{ \pm}(\mathbf{k}) \exp \left(\frac{\mp z}{\tau(\mathbf{k})\left|\mathbf{v}_{z}(\mathbf{k})\right|}\right)\right] .
$$

Here signs \pm refer to whether the $z$-component of the electron velocity is positive or negative. The coefficients $A^{ \pm}$are determined from matching the boundary conditions at the interfaces and outer boundaries in terms of reflection and transmission probabilities and will be considered below. The current density can be obtained from Eq. (12.5).

We note that the solution of the Boltzmann equation takes the form of equation (12.11) only for the CIP geometry which we consider in this section. In this case the current and applied field can be assumed to be uniform within the plane of the multilayer. For the CPP geometry the electric field is position- and spin-dependent because magnetic multilayers are inhomogeneous in the direction of the 
electric current ${ }^{20}$ and, therefore, equation (12.11) does not hold. This point will be further discussed in the next section.

Up to this point we have not specified what is the band structure of the system under consideration. The above derivations are valid for the multiband electronic structure (assuming that the band index is included in $\mathbf{k}$ ) and can be applied for the calculations of conductivity and GMR within the semiclassical approximation in this general case. This will be discussed in section 16 Below we consider a free-electron model.

Within a free-electron model the band structure of a magnetic multilayer or a thin film is described using a single parabolic band which is independent of the spin direction. The complicated electronic structure of the transition metals is therefore significantly simplified by neglecting contribution from the $d$ bands and their strong hybridization with the $s p$ bands. Within the freeelectron approximation the expression for the conductivity per spin, which can be found from Eq.(12.5) by integrating over the film thickness, is simplified (e.g., ref.104):

$$
\sigma=\frac{1}{d} \sum_{i}^{N}\left\{\frac{d_{i}}{\rho_{i}}-\frac{3}{4} \frac{\lambda_{i}}{\rho_{i}} \int_{0}^{1} d \mu\left(1-\mu^{2}\right) \mu\left[A_{i}^{+}(\mu)\left(1-e^{-\frac{d_{i}}{\lambda_{i} \mu}}\right)+A_{i}^{-}(\mu)\left(1-e^{\frac{d_{i}}{\lambda_{i} \mu}}\right)\right]\right\} .
$$

Here we have assumed for simplicity that the relaxation time is independent of $\mathbf{k}$ and introduced the layer-dependent mean free paths $\lambda_{i}=\tau_{i} \mathrm{~V}_{\mathrm{F}}$. In Eq. (12.12) $\mu$ refers to the cosine of the momentum perpendicular to the interfaces, $d$ is the total thickness of the multilayer, $d_{i}$ and $\rho_{i}$ are the thickness and the resistivity of the metal layer $i$, and, as before, we omit spin indices. The first term in this expression gives the conductivity if the various layers were carrying the electric current in parallel. The second term is responsible for finite size effects. The coefficients $A^{ \pm}$can be found using the boundary conditions

Fuchs and Sondheimer applied the semiclassical free-electron model to the conductivity of a homogeneous nonmagnetic thin film. ${ }^{84,85}$ The boundary conditions for the film were determined using the following arguments. If the film is placed at $0<z<d$, the distribution function at $z=0$ must have no electrons with $\mathrm{v}_{z}>0$ other than those specularly reflected from the surface because there are no electrons outside the film. Therefore, defining a fraction $p$ of the electrons which are specularly reflected, the boundary condition at $z=0$ is

$$
g^{+}\left(0, \mathrm{v}_{z}\right)=p g^{-}\left(0, \mathrm{v}_{z}\right)
$$

Similarly at the opposite side of the film, i.e. at $z=d$, the electrons with $\mathrm{v}_{z}<0$ could only be those which are specularly reflected from the boundary:

$g^{-}\left(d, \mathrm{v}_{z}\right)=p g^{+}\left(d, \mathrm{v}_{z}\right)$.

In the case $p=1$, which corresponds to perfect reflection from the boundaries, the conductivity of the film is identical to the conductivity of an infinite homogeneous metal ${ }^{105}$ If $p<1$, a fraction of electrons, $(1-p)$, is scattered diffusively. The case of $p=0$ corresponds to perfectly diffuse scattering, when all the reflected electrons lose memory of their velocity. In the presence of diffuse scattering the conductivity of a thin film decreases with the film thickness. The effect is significant if the film thickness is reduced down to become comparable to the mean free path, i.e. $d \sim \lambda=\mathrm{v}_{\mathrm{F}} \tau$. For thicker films the current density is close to the bulk value in the center of the film, but lowered in the vicinity of the boundaries at distances of the order of $\lambda$.

In the case of layered structures, apart from the probability $p$ for specular reflection at the outer boundaries, additional boundary conditions at the interfaces are required. ${ }^{106}$ These boundary conditions can be imposed by assuming that the electrons are coherently transmitted with probability $T$, coherently reflected with probability $R$, or diffusely scattered with probability $D$ at the interface. The electrons which are diffusely scattered are assumed to be simply lost so that the transmission and reflection probabilities are related by the expression $D=1-T-R$. Thus, at the interface between layers 1 and $\mathrm{i}+1$,

$$
g_{i+1}^{+}=T g_{i}^{+}+R g_{i+1}^{-},
$$$$
g_{i}^{-}=T g_{i+1}^{-}+R g_{i}^{+}
$$

Carcia and Suna ${ }^{106}$ used these boundary conditions to treat the conductance in nonmagnetic multilayers, assuming for simplicity that the electrons can be transmitted or scattered, but cannot be specularly reflected, i.e. $R$ was set equal to zero.

Camley and Barnas ${ }^{107}$ generalized this semiclassical free-electron model to treat GMR in magnetic multilayers and spin valves by assuming that the electric current is spin-dependent and is carried in parallel by the up-spin and down-spin electrons. Within their model scattering in the bulk ferromagnetic layers is treated by introducing spin-dependent relaxation times (or equivalently spindependent mean free paths which enter the expression for the conductivity (12.12)). Scattering at the interfaces is taken into account by assuming spin-dependent transmission coefficients in the boundary conditions (12.15) and (12.16), i.e. $T^{\uparrow} \neq T^{\downarrow}$, specular reflection at the interfaces being neglected Using this semiclassical free-electron model the conductivity and GMR in magnetic multilayers an spin valves can, therefore, be analysed in terms of a number of phenomenological parameters such as spin-dependent mean free paths, spin-dependent transmission probabilities at the interfaces and specular reflection coefficients at the outer boundaries. This model has been extensively used for calculations of GMR and interpreting experimental data. ${ }^{5,75,104,107-110}$ Below, we discuss the main predictions for GMR, which follow from this semiclassical free-electron model.

The magnitude of GMR decreases monotonically with increasing ratio of the thickness of the nonmagnetic spacer layer $d_{N M}$ to the mean free path in the spacer layer $\lambda_{N M}$, similar to that displayed in Figs.11,12. There are two contributions to the decrease of the GMR ratio $\Delta R / R \equiv \Delta \rho / \rho_{P}=\Delta \sigma / \sigma_{A P}$ : the first one contributes to the drop of $\Delta \sigma=\sigma_{P}-\sigma_{A P}$, whereas the second one results in the increase of $\sigma_{A P}$. The drop of $\Delta \sigma$ reflects the reduction in the number of electrons, which can reach an opposite FM/NM interface before being scattered within the spacer layer. Asymptotically, the decrease of $\Delta \sigma$ is exponential with a characteristic decay length equal to the mean free path in the spacer layer, i.e. $\exp \left(-d_{N M} / \lambda_{N M}\right)$. However, this asymptotic regime is reached only for $d_{N M}>>\lambda_{N M}$, because according to formula (12.12) the conductance may be expressed as an integral over an exponential with respect to various incidence angles of the electrons. This asymptotic regime does characterize those multilayers in which $\mathrm{Cu}$ (or other noble metals) is used as the spacer layer. This is due to the relatively large mean free path in $\mathrm{Cu}$, typically of the order of $20 \mathrm{~nm}$ at room temperature as is estimated from the Drude formula. The regime which is relevant to these experiments is characterized by a more complicated variation of $\Delta R / R$ than a simple $\exp \left(-d_{N M} / \lambda_{N M}\right)$. However, as was demonstrated in section 5 , a reasonable description can be obtained by introducing an effective scattering length $l_{N M}$ according to Eq. (3.1). Another contribution to the decrease of GMR as a function of $d_{N M}$ comes from the shunting current within the spacer layer, which leads to an increase of $\sigma_{A P}$ approximately in a linear fashion with $d_{N M}$. If bulk scattering is negligible compared to the scattering at the interfaces, then the change in $\Delta \sigma$ with $d_{N M}$ will be small. In this case the shunting current becomes the dominant contribution and $\Delta \sigma / \sigma_{A P}$ drops as $1 / d_{N M}$

According to the semiclassical free-electron model the variation of GMR as a function of the thickness of the magnetic spacer layer is different depending on whether the bulk or interface spindependent scattering is dominant. In the case of bulk scattering the GMR ratio exhibits a maximum a a certain thickness similar to that in Fig.13. This can be seen from the calculated results presented by the solid lines in Fig.20, which display the magnitude of GMR as a function of the ferromagnetic layer thickness $d_{F M}$ for multilayers with various number of FM/NM bilayers. ${ }^{109}$ In this calculation bulk spin-dependent scattering is introduced through the spin-dependent mean free paths in the ferromagnetic layers, namely $\lambda_{F M}^{\uparrow}=12 \mathrm{~nm}$ and $\lambda_{F M}^{\downarrow}=0.6 \mathrm{~nm}$, and diffuse scattering is assumed at the 
outer boundaries. As is evident from this figure, the position of the GMR maximum shifts towards lower FM layer thicknesses with increasing the number of bilayers $N$. The presence of the GMR maximum and its shift with $N$ is a direct consequence of the bulk spin-dependent scattering and the diffuse scattering at the outer boundaries. In the case of a small number of bilayers, e.g., for the trilayer structure with $N=1$, the position of the GMR maximum is related to the long mean free path in the FM layer, so that $d_{F M} \sim \lambda_{F M}^{\uparrow}$. At this FM layer thickness the up-spin electrons are able to contribute to the conduction before being diffusely scattered at the outer boundaries. On the othe hand, in the case of a large number of the FM/NM bilayers, the position of the GMR maximum is determined by the shorter of the two mean free paths, so that $d_{F M} \sim \lambda_{F M}^{\downarrow}$. This is because for large $N$ the scattering at the outer boundaries becomes unimportant so that the maximum value of GMR wil be obtained when the ferromagnetic layer thickness is sufficient to provide scattering of the downspin electrons.

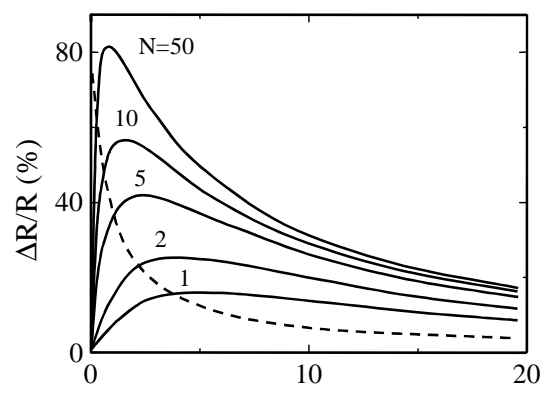

FM layer thickness (nm)

Fig.20 Magnetoresistance versus ferromagnetic layer thickness in (FM/NM) $\mathrm{NM}$ multilayers for bulk (the solid lines) and interface (the dashed line) scattering as calculated using the semiclassical free-electron model. $\lambda_{N M}=20 \mathrm{~nm}, d_{N M}=20 \mathrm{~nm}$, and diffuse outer boundary scattering are assumed in the calculation. The other parameters are set as follows: $N$ is varied, $\lambda_{F M}^{\uparrow}=12 \mathrm{~nm}, \lambda_{F M}^{\downarrow}=0.6 \mathrm{~nm}, T^{\uparrow}=T^{\downarrow}=1$ for bulk spin-dependent scattering and $N=\infty, \lambda_{F M}^{\uparrow}=\lambda_{F M}^{\downarrow}=0.6 \mathrm{~nm}, T^{\uparrow}=1, T^{\downarrow}=0.1$ for interface spin-dependent scattering. After
Dieny. ${ }^{104,109}$

In the case of interface spin-dependent scattering GMR decreases monotonically as a function of the FM layer thickness. This can be seen from the dashed line in Fig.20, which is calculated for the multilayer with an infinite number of repetitions $N$ by introducing spin-dependent transmission coefficients at the FM/NM interfaces, i.e. $T^{\uparrow}=1$ and $T^{\downarrow}=0.1{ }^{104}$ The decrease of GMR reflects the fact that the bulk scattering in the ferromagnetic layers is assumed to be spin-independent and therefore, increasing the FM layer thickness enhances the relative contribution of this type of scattering. When $d_{F M}$ becomes much longer that the mean free path in the FM layer, $\lambda_{F M}$, GMR is inversely proportional to $d_{F M}$, i.e. $\propto \lambda_{F M} / d_{F M} .{ }^{108}$ This dependence can be explained by the argument that only those electrons which leave the FM region of thickness $\lambda_{F M}$ adjacent to the interface have a sufficiently high probability not to be scattered within this FM layer and, therefore, reach the opposite interface. The rest of the FM layer is inactive and serves only as a shunt, which reduces GMR inversely proportional to $d_{F M}$.

The semiclassical model predicts that with increasing number of FM/NM bilayers within a multilayer the value of GMR increases until it reaches saturation, which is similar to that found experimentally and presented in Fig.17a. This tendency can also be seen in Fig.20, according to which at a fixed value of the FM layer thickness the increment of the GMR growth decreases for larger $N$. This behaviour of GMR versus $N$ is due to the diffuse scattering at the outer boundaries of the multilayer. As has already been explained in section 9 , if the longest mean free path is larger than the total thickness of the multilayer, then the diffuse outer-boundary scattering reduces the conductivity of the "good" spin channel and hence effects GMR negatively. The magnetoresistance ratio become independent of the number of bilayers when the total thickness of the multilayer is much larger than the longest mean free path.

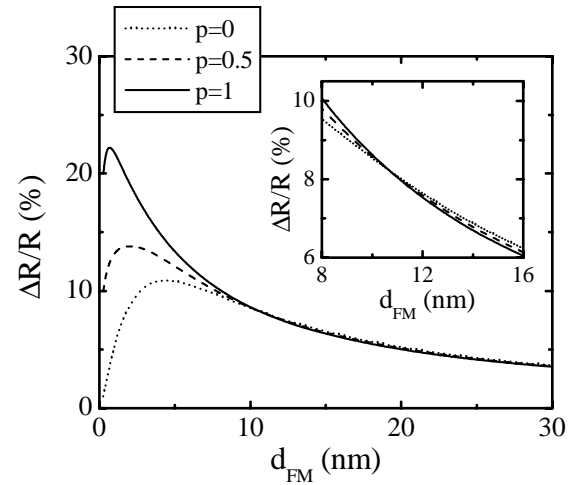

Fig.21 The effect of specular scattering at the top outer boundary of a FM/NM/FM trilayer as calculated from the semiclassical free-electron model. The magnitude of GMR is plotted as a function of the top FM layer thickness for various probabilities of specular reflection $p$ at the top outer boundary. Bulk spin dependent scattering, $T^{\uparrow}=T^{\downarrow}=1$ and $\lambda_{F M}^{\uparrow} / \lambda_{F M}^{\downarrow}=10$, and specular reflection at the bottom outer boundary, $p_{\text {botom }}=1$, are assumed in the
calculation. The other parameters of the model are set as follows: $d_{F M-\text {-bottom }}=2 \mathrm{~nm}$, $d_{N M}=2 \mathrm{~nm}, \rho_{F M}=15 \mu \Omega \mathrm{cm}$, and $\rho_{N M}=6 \mu \Omega \mathrm{cm}$. After Bailey. ${ }^{111}$

Increasing the specular scattering at the outer boundaries strongly enhances GMR in FM/NM/FM trilayers, provided that the FM layers are not too thick. This effect is evident from Fig.21, which shows the calculated magnetoresistance as a function of the top FM layer thickness in the spin valve with varied probability of specular scattering $p$ at the top outer boundary of the trilayer. ${ }^{111}$ Bulk spinwith varied probability of specular scattering $p$ at the top outer boundary of the trilayer. ${ }^{11}$ Bulk spin-
dependent scattering in the ferromagnets and specular reflection at the bottom outer boundary simulating a $\mathrm{NiO}$ pinning layer are assumed in this calculation. The enhancement of GMR with the increasing amount of specularity $p$ is due to the stronger specular scattering from the top surface, which unlike diffuse scattering reflects electrons back allowing them to cross the spin valve many times, thereby increasing spin-filtering effects. Note that the optimum thickness of the FM layer at which the maximum GMR is observed also depends on $p$ and decreases with top surface specularity $p$, which is similar to what was found for the multilayers with an increasing number of FM/NM 
bilayers (Fig.20). This is not surprising because, as has been recognized by Barnas et al.., a multilaye with an infinite number of periods can be simulated by considering a bilayer with specular scattering at the outer boundaries in which the FM layer thickness is taken as half the actual one. At larger FM layer thicknesses the GMR ratio for the specular top surface becomes lower than the GMR ratio for the diffuse-scattering top surface. As is seen from the insert in Fig.21, the crossover occurs at $d_{F M}$ $\approx 10 \mathrm{~nm}$. This is a consequence of the current shunting which becomes dominant over GMR enhancement. Indeed, when the FM layer thickness is larger than the longest mean free path within this layer the specular-reflected electrons are not able to reach the spacer layer and instead contribute to the shunting current.

The semiclassical free-electron model predicts a linear variation in the conductance as a function of $\cos \theta$, where $\theta$ is the angle between the magnetizations of the two ferromagnetic layers in a spin valve. When the magnetic moments of the FM layers are not aligned, the momentum transfer between the up- and down-spin conduction channels is determined by the transmission coefficients which are given by $T_{\uparrow \uparrow}=T_{\downarrow \downarrow}=\cos ^{2}(\theta / 2)$ and $T_{\uparrow \downarrow}=T_{\downarrow \uparrow}=\sin ^{2}(\theta / 2) .{ }^{107}$ Using these expressions and assuming diffuse scattering at the outer boundaries and no specular reflection at the interfaces, it can be shown that the conductance of the trilayer, $\Gamma$, varies in a linear fashion with $\cos \theta,{ }^{27}$ i.e.

$\Gamma(\theta)=\Gamma_{A P}+\left(\Gamma_{P}-\Gamma_{A P}\right)(1-\cos \theta) / 2$

This result is to first order in the GMR ratio equivalent to expression (11.3), according to which the resistance varies linearly with $\cos \theta$. However, the difference between these two descriptions can become sizeable for those systems with relatively large values of GMR (see also section 11).

We see that the semiclassical free-electron model predicts correctly a number of importan features of GMR which are observed experimentally (section III). For example, it qualitatively explains the variation of GMR versus ferromagnetic and nonmagnetic layer thickness, the effect of specular/diffuse scattering at the outer boundaries, the enhancement of GMR with the increasing number of repetitions within a multilayer, and the angular variation of the conductance in spin valves. The great advantage of this model is the ease of application to a particular layered system, which The great advantage of this model is the ease of application to a particular layered system, which
allows understanding qualitative trends in the transport properties. However, as was mentioned above the semiclassical free-electron model ignores the realistic band structure of the multilayer and, therefore, can not be applied for a quantitative description of GMR. Although much experimental dat can be fitted well using the semiclassical free-electron model, the parameters, which are extracted from the fitting, should be treated with caution. For example, Camley and Barnas ${ }^{107}$ have found that in order to account for the increase in GMR from room temperature to liquid helium temperature they had to use a mean free path of $600 \mathrm{~nm}$ at $4.2 \mathrm{~K}$, which is unrealistically long even for the MBE-grown thin films.

A qualitative failure of the semiclassical free-electron model to describe consistently in-situ conductance experiments in $\mathrm{NiO} / \mathrm{Co} / \mathrm{Cu} / \mathrm{Co}$ spin valves was demonstrated recently by Bailey al. ${ }^{112,113}$ They found striking features in the experimental thickness-dependent conductance which is displayed in Fig.22a. As is evident from the figure, addition of about 1 monolayer of Co to $\mathrm{NiO} / \mathrm{Co} / \mathrm{Cu}$ surface causes the net film conductance to decrease. The reverse case of $\mathrm{Cu}$ on $\mathrm{NiO} / \mathrm{Co}$ shows a strong positive curvature of the conductance, indicating a reduction of the conductivity in $\mathrm{Cu}$ near the interface with Co. Detailed microstructural characterization using in-situ Auger electron spectroscopy and $e x$-situ X-ray diffraction measurements indicated that the defect concentration doe not vary noticeably as a function of thickness. These microstructural measurements suggest that the bulk scattering parameters $\rho$ and $\lambda$ should be considered to be constant within each layer, and that the surface scattering parameter $p$ does not change between the layers. Under these constraints, it appears to be impossible to fit even qualitatively the highly asymmetric scattering behavior measured during the formation of $\mathrm{Co} / \mathrm{Cu}$ versus $\mathrm{Cu} / \mathrm{Co}$ interfaces. As can be seen from Fig.22b, depending on the choice of the interfacial transmissivity parameter $T$ the thickness-dependent conductance either do not display any conductance step at the interfaces or display a step at both $\mathrm{Co} / \mathrm{Cu}$ and $\mathrm{Cu} / \mathrm{Co}$ interfaces, neither being observed experimentally. As we will see in section 17, calculations incorporating a realistic band structure resolve the observed inconsistency between the free-electron model and the experiments.
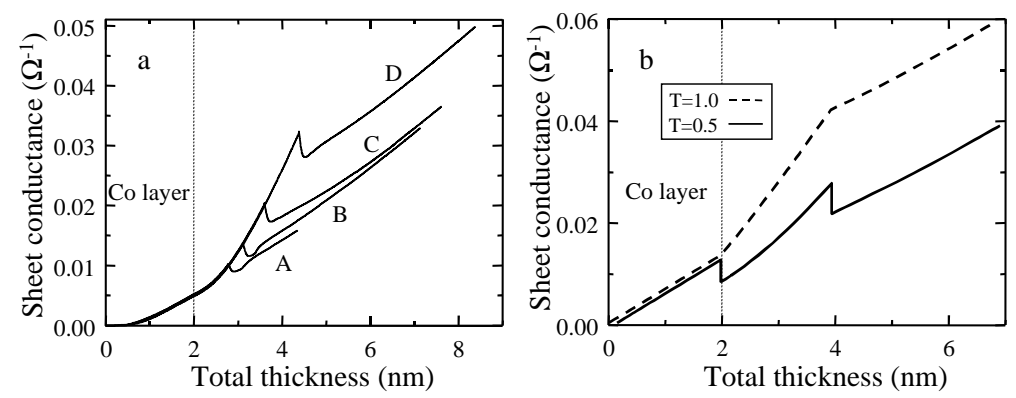

Fig.22 Experimental (a) and calculated (b) thickness-dependent conductance of $\mathrm{NiO} / \mathrm{Co}(2 \mathrm{~nm}) /$ $\mathrm{Cu}\left(t_{\mathrm{Cu}}\right) / \mathrm{Co}(4 \mathrm{~nm})$ spin valves. (a): Conductance is measured $i n$-situ during the deposition of the spin valves with various thickness of the Cu layers: $d_{\mathrm{Cu}}=5.8 \mathrm{~nm}(\mathrm{~A}), 1.1 \mathrm{~nm}(\mathrm{~B}), 1.6 \mathrm{~nm}$ (C), $2.3 \mathrm{~nm}$ (D). The position of the interface with the bottom Co layer is the same for all the samples and is marked by the vertical line. Note the strong deviations from linearity in the vicinity of the interfaces: a drop in film conductance for $\mathrm{Co}$ on $\mathrm{Cu}$ and positive curvature for $\mathrm{Cu}$ on $\mathrm{Co}$. After Bailey et al. ${ }^{12}$ (b): Calculations are performed using a free-electron semiclassical model using parameters which provide a best fit of the data in Fig.21a. Interface scattering $(T<1)$ must be introduced to produce the conductance drop observed during deposition of $\mathrm{Co}$ on $\mathrm{Cu}$, producing a complementary drop for $\mathrm{Cu}$ on Co which is not
observed. After Bailey et al. ${ }^{113}$

Some of the band structure effects can be captured within an extended free-electron model, whic has been proposed by Hood and Falicov. ${ }^{14}$ They introduced layer- and spin-dependent effective masses and the relevant band fillings. They then studied the effect of specular scattering from the resulting potential steps at the interfaces. Unfortunately, the number of free parameters in such a phenomenological model is so large that the analysis of the experimental data in terms of this model becomes uncontrolled.

\section{Quantum-mechanical theory}

In addition to the lack of an accurate description of the electronic structure, the semiclassical freeelectron model suffers from the inability to describe quantum effects, which become important at small film thicknesses. The confinement of electrons in a thin film leads to a discretization of the energy levels. The corresponding quantum effects become observable when the typical spacing $\delta E$ between the energy levels near the Fermi energy becomes larger than the level broadening $\hbar / \tau$ arising from various scattering mechanisms. ${ }^{115}$ Since $\delta E \sim \hbar \mathrm{v}_{F} / d$, where $d$ is the film thickness, the condition for observing quantum size effects is $d<\lambda$, where $\lambda$ is the mean free path. The failure of the semiclassical theory becomes apparent if one considers the conductivity of a thin film with diffusively-reflecting surfaces. ${ }^{116}$ In the limit when the mean free path becomes much longer than the film thickness the conductivity tends to infinity, implying that in the absence of bulk scattering the 
scattering by the rough surfaces induces no dissipation of electrical current. This unphysical result is a direct consequence of ignoring quantum size effects within the semiclassical theory. ${ }^{11}$

In order to resolve this deficiency of the semiclassical model a quantum-mechanical approach to electronic transport is required. There are several different quantum-mechanical formulations of transport theory which include those of Kubo, ${ }^{118}$ Landauer $^{119}$ and Keldysh. ${ }^{120}$ The Kubo (linear response) formalism considers the electronic transport in a disordered metallic system as a linear response to an applied electric field. ${ }^{121}$ The Landauer formalism describes the conductance from the response to an applied electric field. ${ }^{121}$ The Landauer formalism describes the conductance from the
point of view of the transmission of electrons through a conductor and is applicable to mesoscopic transport. ${ }^{122}$ The Keldysh (non-equilibrium Green's function) formalism is conceptually more complicated than the Kubo and Landauer formalisms, but is more general because it provides a description of the quantum transport in the presence of dissipative interactions. ${ }^{122,123}$ All these theories can be used for calculations of GMR within a quantum-mechanical approach. In the present review we outline basic principles of the Kubo theory, which is the most widely used for the treatment review we outline basic principles of the Kubo theory, which is the most widely used fo
of GMR (for a detailed formulation of the linear response theory see, e.g., ref.121).

The starting point of the Kubo formalism is the density matrix. The density matrix is the quantum-mechanical operator, which describes the statistical properties of a quantum-mechanical system. It is the analogue of the distribution function within the semiclassical theory, which we have discussed in section 12 . The density matrix $\rho_{t}$ satisfies the quantum-mechanical equation of motion

$$
i \hbar \frac{d \rho_{t}}{d t}=\left[H_{t}, \rho_{t}\right]
$$

where $H_{t}$ is the Hamiltonian of the system and [,] denotes a commutator. This equation describes the evolution of the system affected by a time-dependent perturbation $U(t)$ due to the applied electric field. We assume that the electric field takes the form $\operatorname{Eexp}(\varepsilon t)$, so that it is uniform in space, is applied at $t=-\infty$ and grows adiabatically to its value $\mathcal{E}$ at $t=0$. The latter is taken into account by an infinitesimal positive $\varepsilon$, so that the limit $\varepsilon \rightarrow 0$ should be taken in the final result. The single-electron Hamiltonian of the system can then be represented by

$$
H_{t}=H+U(t)=H+e \mathcal{E} \cdot \mathbf{r} e^{\varepsilon t},
$$

where $H$ is the time-independent Hamiltonian of the unperturbed system.

Equation (13.1) is the quantum-mechanical analogue of the semiclassical equation (12.1). It describes the time evolution of the system. Initially, i.e. at $t=-\infty$, the system is at equilibrium and is characterized by the unperturbed density matrix $\rho$, according to the Fermi-Dirac distribution $\rho=\left[e^{\left(H-E_{F}\right) / k T}+1\right]^{-1}$. Due to the applied electric field (13.2) the system adiabatically follows the perturbation. Within the Kubo formalism we are looking for the solution of the equation (13.1) to first order with respect to the applied electric field. We can, therefore, represent the density matrix as $\rho_{l}=\rho+\delta \rho(t)$, where $\delta \rho(t)$ is a small time-dependent deviation from the equilibrium Fermi-Dirac distribution $\rho$ due to the applied electric field. The linearized equation for the density matrix (13.1) then takes the form

$$
i \hbar \frac{d \delta \rho(t)}{d t}=[H, \delta \rho(t)]+[U(t), \rho] .
$$

Now we rewrite this operator equation in terms of the matrix elements by introducing a basis of eigenstates $|\alpha\rangle$ of the unperturbed Hamiltonian $H$. The equilibrium density matrix $\rho$ has the same eigenstates as $H$ so that

$$
\rho_{\alpha \beta}=\delta_{\alpha \beta} \frac{1}{e^{\left(E_{\alpha}-E_{F}\right) / k T}+1}=\delta_{\alpha \beta} f\left(E_{\alpha}\right),
$$

where $f\left(E_{\alpha}\right)=\left[e^{\left(E_{\alpha}-E_{F}\right) / k T}+1\right]^{-1}$ is the Fermi-Dirac distribution function and $E_{\alpha}$ is an eigenvalue of $H$. The operator $\mathbf{r}$ which enter the term $[U(t), \rho]$ through equation (13.2) is non-diagonal in the $|\alpha\rangle$ representation. It is convenient to represent the matrix elements of $\mathbf{r}$ in terms of the matrix elements of the velocity operator $\mathbf{v}$ using the relation $i \hbar \mathbf{v}=[\mathbf{r}, H]$, where we can use $H$ instead of $H_{t}$ by neglecting high order terms. Taking this into account we find:

$$
[U(t), \rho]_{\alpha \beta}=i \hbar e \mathcal{E} \cdot \mathbf{v}_{\alpha \beta} \frac{f\left(E_{\alpha}\right)-f\left(E_{\beta}\right)}{E_{\alpha}-E_{\beta}} e^{\varepsilon t} .
$$

The time dependence of $\delta \rho(t)$ is determined by the perturbation (13.5), so that $\delta \rho(t)=\delta \rho \exp (\varepsilon t)$. Using equations (13.4-13.6) we find the solution for the density matrix at $t=0$

$$
\delta \rho_{\alpha \beta}=\frac{i \hbar e \mathcal{E} \cdot \mathbf{v}_{\alpha \beta}}{E_{\beta}-E_{\alpha}+i \varepsilon} \frac{f\left(E_{\alpha}\right)-f\left(E_{\beta}\right)}{E_{\alpha}-E_{\beta}} .
$$

This solution can now be used for calculating the electrical current. For a spatially homogeneous system the current-density operator is defined by $\mathbf{j}=-e \mathbf{v} / \Omega$, where $\Omega$ is the total volume of the system. We need to calculate the expectation value of $\mathbf{j}$ which is determined by $\langle\mathbf{j}\rangle=\operatorname{Tr}(\mathbf{j} \delta \rho)$, where we have used the fact that $\operatorname{Tr}(\mathbf{j} \rho)=0$, as in equilibrium there is no net current in the system. Using the definition of the conductivity (12.8) and taking the limit $\varepsilon \rightarrow 0$ we obtain

$$
\sigma^{\mu v}=\frac{e^{2} \pi \hbar}{\Omega} \sum_{\alpha \beta} \mathrm{v}_{\alpha \beta}^{\mu} \mathrm{v}_{\beta \alpha}^{v} \delta\left(E_{\alpha}-E_{\beta}\right)\left[-f^{\prime}\left(E_{\alpha}\right)\right]
$$

where we have taken into account that $\operatorname{Re} \lim _{\varepsilon \rightarrow 0} \frac{i}{x+i \varepsilon}=\pi \delta(x)$ and used the fact that as $E_{\alpha} \rightarrow E_{\beta}$, which is required by the $\delta$-function, $\left[f\left(E_{\alpha}\right)-f\left(E_{\beta}\right)\right] /\left(E_{\alpha}-E_{\beta}\right) \rightarrow f^{\prime}\left(E_{\alpha}\right)$. Using the identity

$$
\delta\left(E_{\alpha}-E_{\beta}\right)=\int_{-\infty}^{\infty} d E \delta\left(E-E_{\alpha}\right) \delta\left(E-E_{\beta}\right)
$$

and taking the zero-temperature limit at which $\left[-f^{\prime}(E)\right]=\delta\left(E-E_{F}\right)$, formula (13.7) for the conductivity can be neatly represented by

$$
\sigma^{\mu v}=\frac{e^{2} \pi \hbar}{\Omega} \operatorname{Tr}\left\{\mathrm{v}^{\mu} \delta\left(E_{F}-H\right) \mathrm{v}^{v} \delta\left(E_{F}-H\right)\right\} .
$$

The above formula for the conductivity would generally depend on the particular type of the disorder responsible for the scattering. In order to obtain a result that is independent of the particula disorder configuration but depends only on average characteristics (e.g., defect density or impurity concentration), one has to perform a configurational average of this expression. In this form the above expression is known as the Kubo-Greenwood formula. ${ }^{12}$

In many cases it is convenient to carry out the configurational averaging using techniques which have been developed for Green's functions (e.g., refs.121,125,126). Therefore it is useful to rewrite the Kubo-Greenwood formula in the following form

$$
\sigma^{\mu v}=\frac{e^{2} \hbar}{\Omega \pi}\left\langle\operatorname{Tr}\left\{\mathrm{v}^{\mu} \operatorname{Im} G\left(E_{F}\right) \mathrm{v}^{v} \operatorname{Im} G\left(E_{F}\right)\right\}\right\rangle .
$$

Here the angular brackets stand for the configurational average and we have introduced the Green's function, which is defined by 


$$
G(E)=\lim _{\varepsilon \rightarrow 0} \frac{1}{E-H+i \varepsilon},
$$

and have taken into account that $\delta(E-H)=-\operatorname{Im} G(E) / \pi$

The general techniques for configurational averaging in disordered homogeneous systems have been described in detail elsewhere, e.g., in refs.121,125,126. Here we briefly summarize the main results. We assume that the total Hamiltonian of the system can be represented by $H=H^{0}+V$, where $H^{0}$ describes the undisturbed periodic system and $V$ is scattering potential due to the defects or impurities. By configurational averaging we replace this system which is characterized by the random non-periodic potential by an effective medium which possess translational invariance. The configurational averaging leads to the renormalization of the Green's function so that

$$
\langle G(E)\rangle=\frac{1}{E-H^{0}-\Sigma(E)},
$$

where $\Sigma$ is the self energy. The above equation can be considered as the definition of $\Sigma$, which is an energy-dependent non-Hermitian operator. Its real part shifts the energy levels of the undisturbed system, whereas the imaginary part characterizes the broadening of the levels due to the finite scattering lifetime. $\operatorname{Im} \Sigma\left(E_{F}\right)$ determines, therefore, the relaxation time $\tau$, which has been introduced within the semiclassical theory (section 12$)$

It follows from equation (13.10) that the conductivity tensor requires an average over the product of two Green's functions, i.e. $\sigma \propto\langle\mathrm{GG}\rangle$. In general, performing this averaging explicitly is complicated problem. This is the reason why very often the conductivity is approximated by the product of the average of the Green's functions, i.e. $\sigma \propto\langle\mathrm{G}\rangle\langle\mathrm{G}\rangle$. By making this approximation one ignores the contribution from the vertex corrections in the linear response formalism. ${ }^{121}$ This approximation is equivalent to neglecting the scattering-in term in the semiclassical theory, which allows the introduction of the relaxation time approximation (section 12). Similar to the relaxation time approximation, the neglect of the vertex corrections in quantum-mechanical linear-response theory is a non-trivial approximation and has to be justified (see ref.121 for a discussion).

We note that the above derivation of the formula for conductivity is valid for a homogeneous system. In this case the current and applied field can be assumed to be uniform so that one can define the conductivity according to equation (12.8). This is the conductivity, which is given by the KuboGreenwood formula (13.10). In a general case of an inhomogeneous system the current density is determined by the non-local conductivity according to

$$
\mathbf{j}^{\mu}(\mathbf{r})=\int d \mathbf{r}^{\prime} \sum \sigma^{\mu v}\left(\mathbf{r}, \mathbf{r}^{\prime}\right) \mathcal{E}^{v}\left(\mathbf{r}^{\prime}\right) .
$$

The electric field $\mathcal{E}(\mathbf{r})$ in this equation is the local electrostatic field, which arises from the application of the potential difference across the sample. As was explained by Levy, ${ }^{20}$ this is an internal field which is not the same as the field applied externally. For inhomogeneous magnetic systems the local internal field is position- and spin-dependent. If the rate at which the electrons are scattered varie from one region to another, then electrical conduction will lead to a spatial redistribution of charge. This charge redistribution in inhomogeneous media results in a nonuniform internal electric field. In magnetic systems electron conduction is spin-dependent. Spin-polarized electric currents in inhomogeneous media lead to a spatial redistribution of spin as well as charge. ${ }^{127}$ This phenomenon is known as spin accumulation or current-driven magnetization. The internal electric field in equation (13.13) may, therefore, be different for different spins. In magnetic multilayers the effect of position- and spin-dependent internal field is very important for perpendicular transport because these eneous in the direction of electric current ${ }^{20}$ For parallel transport, however, the internal electric field is a constant, because these layered systems are homogeneous in the plane of the layers. The Kubo-Greenwood formula (13.10) can, therefore, be used for the treatment of CIP GMR.
The quantum-mechanical model for GMR was first introduced by Levy et al. ${ }^{128}$ They used the Kubo formalism to calculate the conductivity of free electrons scattered by a spin-dependent potentia

$$
V(\mathbf{r})=\sum\left(v_{a}+j_{a} \mathbf{m} \cdot \boldsymbol{\sigma}\right)\left(\mathbf{r}-\mathbf{r}_{a}\right),
$$

which is produced by random-point scatterers. In the above formula $\mathbf{r}_{\mathbf{a}}$ is a random position of the scatterer, $\mathbf{m}$ is a unit vector in the direction of magnetization, $\sigma$ is the Pauli matrix, $v_{\mathrm{a}}$ and $j_{\mathrm{a}}$ characterize the strength of the spin-independent and spin-dependent contribution to the scattering potential respectively, which may be different for different layers and interfaces. Performing the configurational averaging in momentum space, Levy et al. derived a simple formula for the local conductivity, which can be expressed in terms of the local spin-dependent scattering rate $\Delta(z)$ as

$$
\sigma(z)=\frac{n e^{2}}{m \Delta(z)},
$$

where $z$ is a coordinate perpendicular to the layers. The local scattering rate $\Delta(z)$ is determined by the appropriate average of the $z$-dependent scattering rates within the different layers. The total CIP conductivity of the multilayer can be found by integrating over the multilayer thickness $L$, i.e.

$$
\sigma=\frac{1}{L} \int_{L} \sigma(z) d z
$$

In the limit of the mean free path being long compared to the layer thickness, $\lambda \gg d$, this mode reduces to the series-resistor model, which was introduced in section 3. In particular, in the case of interface scattering the result for GMR is given by formula (3.5), in which the asymmetry parameter $\alpha$ is determined by the interfacial scattering parameters in equation (13.14), namely $j_{\text {int }} / v_{\text {int }}=(\sqrt{\alpha}-1) /(\sqrt{\alpha}+1)$. In the case of bulk scattering the value of GMR can be found from formula (3.6), in which $\alpha$ is determined by bulk scattering parameters in equation (13.14), namely $j_{\text {bulk }} / v_{\text {bulk }}=(\sqrt{\alpha}-1) /(\sqrt{\alpha}+1)$.

The same model of free electrons scattered by spin-dependent potential (13.14) was used by Camblong et al. ${ }^{129,130}$ to describe GMR within a real-space approach. According to Camblong ${ }^{130}$ the real-space approach works better because it avoids the local approximation for the conductivity which was used in the momentum space approach of ref.128. Solving equation (13.12) within a weak scattering approximation and in the dilute limit of impurity concentration, Camblong et al. derived expressions for the non-local conductivity for both the CIP and CPP geometries. They found that the semiclassical approach for multilayers and the real-space quantum theory produce the same magnetotransport properties, provided the effect of quantum interference and quantum-size effects are neglected. A similar conclusion was arrived at by Vedyayev et al. ${ }^{131}$ who used the real-space quantum-mechanical approach for the conductivity of free electrons affected by bulk spin-dependen scattering in spin valves. ${ }^{132}$

The quantum models of Levy et al. ${ }^{128}$ and Camblong and Levy ${ }^{129,130}$ have been compared with the "exact" solution for the conductivity by Zhang and Butler. ${ }^{133}$ Similar to refs.128-130 they used the model of free electrons with random point scatterers and calculated the position-dependent conductivity without the vertex corrections. However, instead of deriving the expression for the selfenergy from a Hamiltonian by configurational averaging, they assumed that the imaginary part of the self-energy is a phenomenological parameter and compared approximations used by the previous authors within the quantum and semiclassical models. Zhang and Butler found that the Camblong and Levy's approach is identical to the Fuchs-Sondheimer theory within the relaxation time approximation and specular boundary conditions at the interfaces. As can be seen from Fig.23a, this approach predicts the total CIP conductivity of a multilayer in good agreement with the exact solution. This fact demonstrates that in the absence of potential discontinuities at the interface 
quantum effects, such as oscillations in the conductivity, are effectively averaged out and the semiclassical solution provides a good approximation for GMR within the free-electron model. It i also clear from Fig.23a that although the model of Levy et al. ${ }^{128}$ predicts the correct thin and thick limits, it does not accurately reproduce the exact results for intermediate film thicknesses. Fig.23b compares the calculated results for GMR within the different models. As is seen from the figure, the model of Levy et al. strongly overestimates GMR, whereas the Camblong and Levy's or semiclassical results are much closer to the "exact" curve.
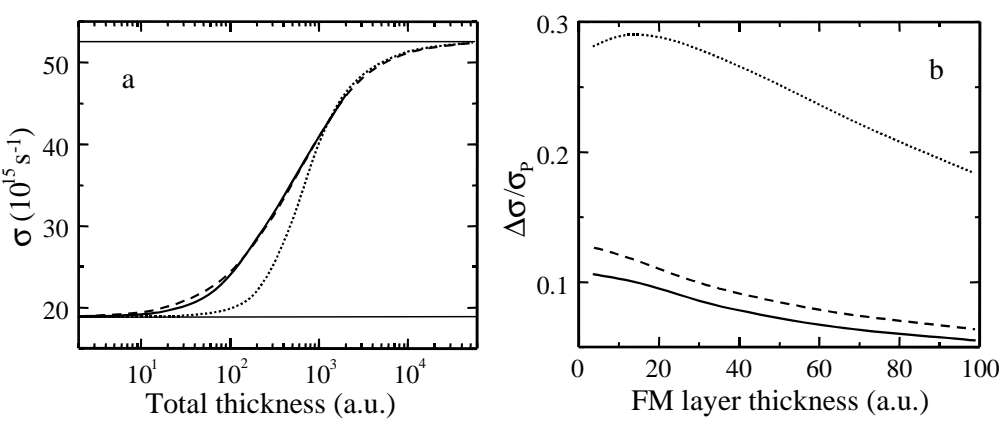

Fig.23 Comparison of "exact" calculations (solid lines) with different free-electron models for GMR, the model of Levy et al. ${ }^{128}$ (dotted lines) and the model of Camblong and Levy ${ }^{129,130}$ (dashed lines). Note that Camblong and Levy's theory is identical to the Fuchs-Sondheimer theory with $p=1$. (a): The calculated conductivity (in units $10^{15} \mathrm{~s}^{-1}=1113 \Omega^{-1} \mathrm{~cm}^{-1}$ ) as a function of the total thickness of a period for a nonmagnetic multilayer with a period of two layers and with mean free paths in each layer of $\lambda_{1}=36 \mathrm{a} . u$. and $\lambda_{2}=360$ a.u. (1a.u. $=0.0529 \mathrm{~nm}$ ). The thickness of the first layer is twice that of the second. The horizontal lines show the limits of thin and thick films. (b): The calculated GMR of a magnetic multilayer as a function of the ferromagnetic $\lambda^{2}=10$ a.u. are introduced to model strong spin-dependent scattering at the interfaces. Other

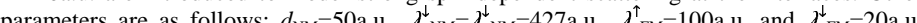

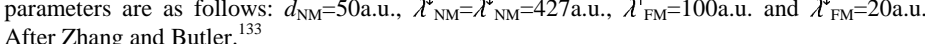

The above quantum models for GMR assume that the origin of GMR lies in spin-dependent scattering potentials at the interfaces or in the bulk metals. By using the free-electron approximation these models ignore the variation of the intrinsic spin-dependent electronic potential of the layered structures. An extension of the free-electron theory for magnetic multilayers to include the intrinsic potential within the Kronig-Penney model has been performed by Zhang and Levy ${ }^{134}$ in the momentum-space approach and Bulka and Barnas ${ }^{135}$ in the real-space approach. They found that the spin-dependent steps at the interfaces could enhance or reduce GMR depending on the parameter characterizing the potential. It was also shown that the GMR phenomenon could occur in structures with no spin asymmetry in the relaxation times, but with a spin-dependent electronic structure (which originates in the above models from introducing a spin-dependent potential within the multilayer). However, quantitative predictions are difficult because of the unknown parameters characterizing the spin-dependent potential. Only a full band structure calculation can predict why potential steps at the interfaces may be important.

The effect of the intrinsic step-like potential on GMR in spin-valve structures was also considered by Vedyayev et al. ${ }^{136}$ and Barnas and Bruynseraede. ${ }^{137}$ They found that their results are strongly influenced by quantum-size effects at small layer thicknesses and predicted oscillations in GMR as a function of the layer thicknesses. Unfortunately, these quantum-size effects in GMR which make the quantum free-electron models conceptually different from the semiclassical free-electron models have not yet been observed. Apart from the quantum-size effects, the variation of CIP GMR as a function of magnetic and non-magnetic layer thickness within the quantum free-electron models qualitatively reproduces semiclassical results. ${ }^{129,133}$ Although quantitatively the predictions of the semiclassical and quantum models might differ, the experimental data can be fitted well using both approaches. ${ }^{131}$ However, neglecting the realistic band structure, which is the grossest approximation inherent in the free-electron models, makes quantitative comparison of these models with experiments unreliable. Including an accurate spin-polarized band structure to the models for GMR will be considered in section $\mathrm{V}$.

\section{Tight-binding models}

A single-band tight-binding model is another simple way to describe the electronic band structure of a metal. Unlike the free-electron theory, the tight-binding theory describes the electronic structure in terms of localized atomic orbitals, which overlap due to the bonding between neighboring atoms. The propagating (Bloch) states, which are responsible for the electronic transport in metals, can be built up from the atomic orbitals by solving the respective Schrödinger equation. The tight-binding approach is especially suited to numerical calculations of the conductance because it discretizes the spatial continuum in terms of atomic cites. A single-band approximation to the tight-binding model is, of course, a strong simplification to the band structure. However, as we will see in section 17, the tight-binding model can be generalized to a multiband description of the electronic structure which makes it a very powerful tool for modeling GMR.

A single-band tight-binding Hamiltonian takes the form

$$
H=\sum_{i}|i\rangle E_{i}\left\langle i\left|+\sum_{i j}\right| i\right\rangle h_{i j}\langle j| \text {, }
$$

where $E_{i}$ are $|i\rangle$ are the on-site atomic energy and the atomic orbital at site $i$ respectively. The tightbinding hopping matrix elements $h_{i j}$ are usually assumed to be non-zero only between nearestneighbor lattice sites. This simple model is quite flexible and allows elaboration. In particular, the Stoner exchange splitting of the spin bands can be included in this model by taking different on-site atomic energies for the up- and down-spin electrons. Disorder can be introduced by assuming randomness in the on-site energies or in the hopping matrix elements. Impurities can be included by putting atoms with their on-site energies, which are different from those of the host atoms. Using thi tight-binding model for studying GMR helps to elucidate the microscopic origin of spin-dependen scattering, an important issue which has not been addressed within the phenomenological freeelectron models described in sections 12 and 13.

As was discussed in the previous section, in order to calculate the transport properties of a disordered metal an appropriate configurational average has to be performed. This configurational averaging can be carried out analytically using the Green's function's methods (e.g., ref.125) within certain approximations, e.g., low density of impurities/defects, weak scattering potentials, and neglecting vertex corrections. The drawback of this approach is that frequently the validity of the approximate solution is difficult to monitor. An alternative approach is to perform the averaging numerically by generating a number of random configurations of disorder/impurities within a sufficiently large cell. An efficient method for calculating conductance using this approach has been sufficiently large cell. An efficient method for calculating conductance using this approach has been
developed in the field of mesoscopic physics. ${ }^{139}$ This method utilizes the discrete nature of the tightbinding model and is based on the Kubo formula and the recursive Green's function technique. The disadvantage of the recursive method is that it is computationally demanding. One is limited to a relatively small cell size, which may lead to artifacts due to the effect of boundary conditions. 
Nevertheless, this method has become a powerful tool for studying GMR, especially within the CPP geometry (see section VI). Below we outline the basic ideas of this approach.

The geometry of the system, which is normally considered for calculating the conductance within the recursive technique, is shown schematically in Fig.24. The sample under consideration, e.g., a magnetic multilayer, is placed between two semiinfinite leads. The sample can be, in general, disordered but the leads are assumed to be perfect. We note that although the system, which is shown in Fig.24, represents an infinite wire, periodic boundary conditions can, if required, be imposed in the transverse direction to built an infinite multilayer. It is assumed that at infinity the leads are connected to reservoirs, which are at thermodynamic equilibrium. The electric current in the system is driven by a small electrochemical potential difference between the reservoirs. Such a formulation of the problem is typical for the Landauer approach to the electronic transport in mesoscopic systems. ${ }^{122}$ It has been proved that the Landauer formalism can be derived directly from the Kubo formalism. ${ }^{140}$ the latter being an efficient method for calculating the conductance within the recursive technique. ${ }^{139}$

(a) CIP geometry

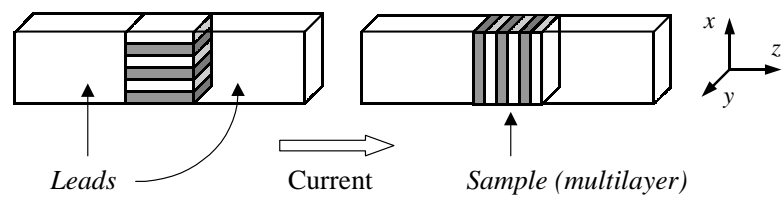

Fig.24 Geometry for the CIP (a) and CPP (b) GMR calculation within the tight-binding recursion Geometry for the CIP (a) and CPP (b) GMR calculation within the tight-binding recursion electric current flows in the $z$ direction.

Within the above formulation the conductance rather than the conductivity (13.10) is the subject of interest. The Kubo formula (13.10) for the zero-temperature conductance $\Gamma$ per spin can be rewritten for the case of a simple cubic lattice with lattice constant $a$ as

$\Gamma=\frac{2 e^{2} \hbar}{\pi a^{2}} \operatorname{Tr}\left\{\mathrm{v} \operatorname{Im} G\left(E_{F}\right) \mathrm{v} \operatorname{Im} G\left(E_{F}\right)\right\}$.

Here $\mathrm{v}$ is the projection of the velocity operator to the direction of current (the $z$ direction in Fig.24) and $G\left(E_{F}\right)$ is the Green's function of the total coupled system, which includes the right and the lef electrodes and the sample, at the Fermi energy $E_{F}$. The Kubo formula (14.2) can be evaluated by "cutting" the system in the transverse direction (i.e. in the $x y$ plane in Fig.24) and calculating the matrix elements of the Green's function and the velocity operator between the atomic planes $l$ and $l+1$. Due to the current conservation condition the result for the conductance is independent of $l$ and the latter can be chosen arbitrarily. The velocity operator can be represented as

$$
\mathrm{v}=\frac{i a}{\hbar} \sum_{i j} h_{i l, j l+1}\{|j, l+1\rangle\langle i, l|-| i, l\rangle\langle j, l+1|\},
$$

where $|i, l\rangle$ is the atomic orbital of atom $i$ in plane $l$ and $h_{i l, j l+1}$ are hopping matrix elements between planes $l$ and $l+1$. Normally it is convenient to take $l$ being the last atomic plane of the sample $L$ so that $L+1$ is the first layer of the right lead. The matrix elements of the Green's function of the total system at planes $L, L+1$ and between them can be evaluated using the recursion technique as described below.

First, one finds the matrix elements of the surface Green's function for the detached semi-infinite leads. A simple algebraic expression can be derived within the single-band tight-binding model and simple-cubic geometry. ${ }^{141}$ In a general case, the surface Green's function can be expressed in terms of the Green's function for the bulk metal, ${ }^{142}$ the latter being calculated in momentum space using standard techniques. Then, the sample is grown by adding atomic layers with impurity atoms distributed randomly, layer by layer, onto the left lead. At every step the Green's function matrix elements between atomic sites in the last added layer are calculated by solving numerically the Dyson equation:

$$
g_{l+1}=\left(H_{l+1}-h_{l+1, l} g_{l} h_{l, l+1}\right)^{-1} .
$$

Here $\mathrm{g}_{l}$ is the Green's function of the left semiinfinite lead with added $l$ layers $(0 \leq l \leq L)$ of the sample (before adding the layer $l+1$ ), $\mathrm{g}_{l+1}$ is the Green's function of the left semiinfinite lead with added $l+1$ layers of the sample (after adding the layer $l+1$ ), $H_{l+1}$ is the Hamiltonian of the added layer $l+1, h_{l l+1}$ is the hopping (bonding) between atoms in the new layer $l+1$ and atoms in the previous layer $l$. Once the sample has been fully-grown, the last layer is bonded to the right lead in order to obtain the Green's function $G\left(E_{F}\right)$ of the full system, which enters the expression for the conductance (14.2). We note that this method gives an exact solution for the given model and geometry and describes the conductance for a particular disorder/impurity configuration. The configurational averaging should be performed numerically by generating a number of random disorder/impurity configurations.

Asano et al. ${ }^{143}$ implemented this approach for studying GMR in a $\mathrm{Fe} / \mathrm{Cr}$ magnetic multilaye within the CIP and CPP geometries. They used a single-band $s$-valent tight-binding model within simple cubic geometry with (001) orientation of atomic planes and constant hopping $h$ between nearest-neighbors (so that $h_{i l, j l+1}=h \delta_{i j}$ in equation (14.3)). They introduced a Stoner exchange splitting $2 J$ of the spin bands in $\mathrm{Fe}$, so the on-site atomic energies of the majority- and minority-spin electrons were equal $E_{F e}^{\uparrow}=E_{F e}-J$ and $E_{F e}^{\downarrow}=E_{F e}+J$ respectively. They assumed that $\mathrm{Cr}$ was non-magnetic with on-site atomic energy chosen to be equal to the minority on-site atomic energies of Fe, i.e. $E_{C_{l}}=E_{\mathrm{Fe}}^{\downarrow}$ Due to this the minority-spin electrons do not experience a potential step (or roughness potential) for the parallel alignment of the magnetizations. Using this model Asano et al. studied the effect of interface roughness and bulk disorder on CIP and CPP GMR. The interface roughness was introduced through substitutional randomness at the interfacial layers. This implied that in the $\mathrm{Fe}$ layer, each atom adjacent to the $\mathrm{Cr}$ layer was replaced by the $\mathrm{Cr}$ atom with a probability $c$. Similarly, in the $\mathrm{C}$ layer each atom adjacent to a Fe layer was replaced by the $\mathrm{Fe}$ atom with the same probability $c$. The bulk disorder was modeled by a random variation of the on-site atomic energies of the $\mathrm{Fe}$ and $\mathrm{C}$ atoms with a uniform distribution of width $\gamma$, which was assumed to be spin-independent and was allowed to vary in the calculations. The resulting conductance was averaged over 100 random configurations of roughness or disorder.

As is evident from Fig.25a, the magnitude of CPP GMR is much larger than the magnitude of CIP GMR and they behave differently as a function of the interface roughness, the measure of which is the intermixing concentration $c$ of the two monolayers forming the interface. Not unexpectedly, within the model considered the interface roughness has a beneficial effect on CIP GMR, because it is the only mechanism of spin-dependent scattering and, therefore, CIP GMR increases with $c$ (the ful squares in Fig.25a). The presence of steps in the electronic potential at the interfaces has little influence on CIP GMR, which is found to be close to zero in the absence of roughness. On the contrary, a sizeable CPP GMR is found in the absence of any roughness, the latter only weakly contrary, a sizeable CPP GMR is found in the absence of any roughness, the latter only weakly
reducing GMR (the open circles in Fig.25a). This is the result of the spin-dependent potential of the multilayer, which effects differently the number of electrons contributing to the conduction for the parallel and antiparallel configurations (see also section 15). Figure 25b shows the magnitude of 
GMR as a function of bulk disorder within the multilayer, which is measured by the width of the distribution in the on-site atomic energies $\gamma$. As is seen from the figure, the value of GMR decrease with increasing $\gamma$ for both the CIP and CPP geometries. This is a direct consequence of the increasing scattering in the "good" minority conduction channel, which reduces the conductance within the parallel configuration of the multilayer.
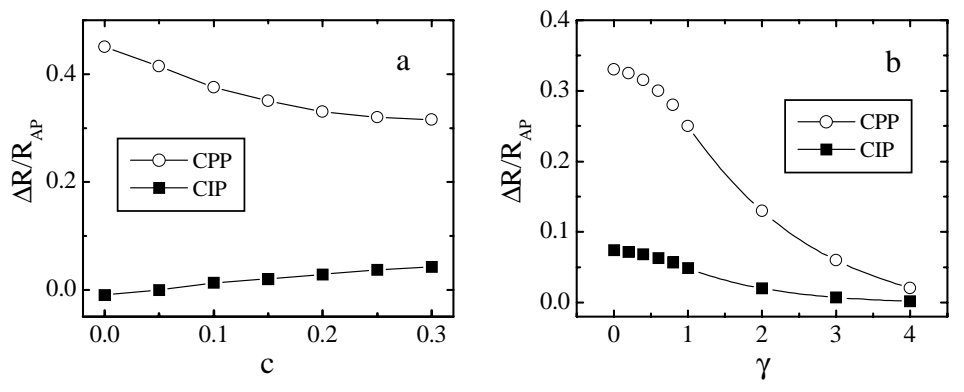

Fig.25 Calculated GMR of $(\mathrm{Fe} / \mathrm{Cr})_{2}$ multilayer within a single band tight-binding model as a function of interface roughness $c$ (a) and bulk disorder $\gamma$ (b). Each metal within the multilayer layer consists of three atomic monolayers of cross section $12 \times 12$ atoms. Roughness is measured in terms of the intermixing concentration $c$ of the two monolayers forming the interface. Disorder is introduced as a random variation in the on-site atomic energy levels of width $\gamma$. The tight-biding parameters in units of the hopping integral $h$ are roughness/disorder configurations. In figure (b) $c$ is fixed at 0.2. Note the definition of GMR as $\Delta R / R_{A P}$. After Asano et ll. $^{143}$

A similar tight-binding model was used by Itoh et al. ${ }^{144}$ who studied the combined effect of roughness and potential barriers at the interfaces on CIP and CPP GMR in magnetic multilayers. Unlike Asano et al..$^{143}$ they used the coherent potential approximation (CPA) ${ }^{126,145}$ to perform their Unlike Asano et al., ${ }^{13}$ they used the coherent potential approximation (CPA)
configurational averaging. Within the CPA the disordered alloy (at the interface) is replaced an effective medium which is characterized by the Hamiltonian $H_{e f f}=H_{0}+\Sigma$, where the non-hermitian selfenergy operator $\Sigma$ is defined by equation (13.12). The approximate solution for $\Sigma$ can be found by the self-consistent condition that the scattering of electrons in the effective medium by any site (a singlesite CPA) or any cell (a single-cell CPA) vanished on average. Itoh et al ${ }^{144}$ used the single-cell CPA,

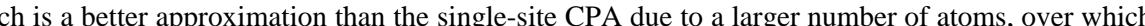
the configurational averaging is performed exactly. Within the single-cell CPA they derived selfconsistent analytic expressions for the conductivity including the vertex corrections. They found tha in the CIP geometry the vertex corrections vanish, whereas in the CPP geometry the vertex corrections do not necessarily vanish. Since the single-cell CPA is computationally expensive, Itoh et al. carried out numerical calculations in the weak-scattering limit for thin metal layers consisting of three atomic monolayers. Their results for GMR are consistent with those predicted by Asano et al. ${ }^{143}$ within the recursive technique. They find that the CPP GMR is larger than the CIP GMR, which is a consequence of the spin-dependent potential steps at the interfaces introduced in their model. The difference between the CPP and CIP geometries decreases strongly with decreasing step size. The interfacial roughness is favorable to CIP GMR but has a small negative effect on the CPP GMR. They also find that the contribution from the vertex correction to the CPP conductance is negligible. Thi result might be the consequence of the small multilayer period, which was used in computations, so that the mean free path is much longer than the layer thickness.

The recursive technique was used by Todorov et al. ${ }^{146}$ for calculating CIP GMR of the $\mathrm{FM} / \mathrm{NM} / \mathrm{FM}$ trilayer to study the effect of band structure and interface roughness within a single-band tight-binding model. There are three major differences between the models used by Todorov et al. and Asano et al. ${ }^{143}$ First, Todorov et al. introduced different hopping integrals for the up-spin and downspin electrons in the ferromagnetic metal. This was supposed to reflect the difference in the majorityand minority-electron dispersions and the density of states at the Fermi energy, which are typical for real ferromagnetic metals. The on-site atomic energies were assumed for simplicity to be the same for all the atoms. Second, the interface roughness was introduced as steps of random length $l_{\text {step }}$ along the trilayer with a correlation length $l_{c o r}$ defined by $l_{c o r}=\left\langle l_{\text {step }}\right\rangle$. This allowed studying the effect of the roughness correlation length (i.e. the step density) on GMR. Third, the calculations were performed for a range of trilayer lengths $L$, and the linear region of the $R$ versus $L$ relation was used to obtain the Ohmic resistivity $\rho=A(d R / d L)$, where $A$ is the trilayer cross section. This eliminated the effect of contact resistances, which affected the value of GMR that are obtained directly from $R$ for a fixed $L{ }^{143}$ The range of $L$, from which $\rho$ was calculated, exceeded the effective mean free path in the trilayer by a factor between 5 and 10 , so that the diffusive regime of conduction is being modeled.
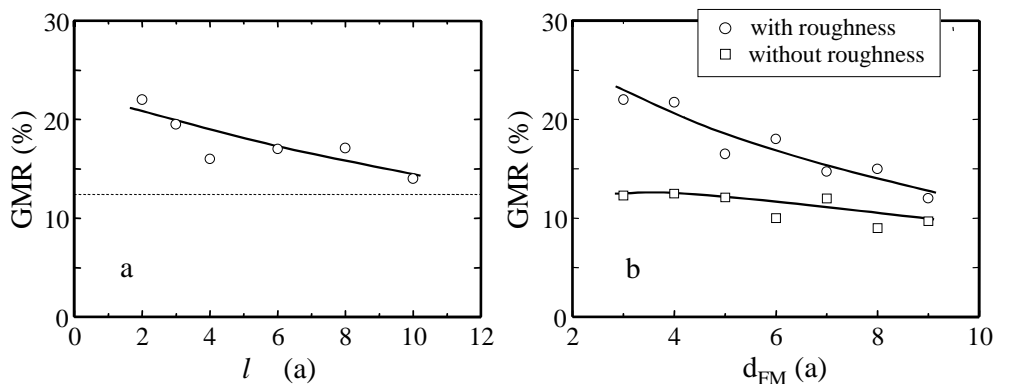

Fig.26 Calculated GMR of a FM/NM/FM trilayer within a single-band tight-binding model as a function of the correlation length of roughness $l_{c o r}$ (a) and as a function of ferromagnetic layer thickness $d_{F M}$ (b). The width of the trilayer is $8 a$ and the NM layer thickeness is $4 a$. The band structure of the FM metal is approximated by the two spin bands with different hopping integrals, namely $h^{\uparrow}=1$ and $h^{\downarrow}=1 / 3$. Other parameters are as follows: $E_{F}=-0.5, h_{N M}=1$, bulk disorder parameter $\gamma=0.5, E_{F M}=E_{N M}=0$, in figure (a) $d_{F M}=4 a$, in figure (b) $l_{\mathrm{cor}}=2 a$. The solid lines are eye guides. After Todorov et al. ${ }^{146}$

Todorov et al. find a sizable CIP GMR in the absence of the interface roughness. The effect arises from the difference in the mean free path and bulk resistivity between the up-spin and th down-spin channels in the magnetic material, which in turn comes from the respective difference in the density of states and the Fermi velocity. GMR decreases with increasing bulk disorder, approaching zero in the limit of the mean free path for both spins becoming smaller than the trilayer thickness. The above results indicate that the spin-dependent band structure, in conjunction with the intrinsic bulk disorder in real systems, is a sufficient condition for GMR. The chemically sharp interfacial roughness generally enhances the effect. The contribution of the roughness, however becomes quantitatively significant only in the limit of sufficiently dense interfacial steps (smal 
correlation length of the roughness), sufficiently weak bulk scattering, and sufficiently thin magnetic layers. This is evident from Figs.26a and 26b. Fig.26a shows GMR as a function of the roughnes correlation length. As is seen from the figure, with increasing correlation length GMR decreases, approaching its value without roughness. These results are consistent with the experimental data by Schad $e$ t al. ${ }^{70}$ on GMR in epitaxial Fe/Cr multilayers discussed in section 7 (see also Fig.14a). Fig.26b shows the dependence of GMR on the magnetic layer thickness. As can be seen from the figure, GMR decreases with increasing $d_{F M}$, but in the case with the roughness, the decrease is faster than in the case without the roughness. This result is consistent with the different behavior of bulk and interface spin-dependent scattering predicted by the semiclassical free-electron model (see section 12).

Although some band structure effects have been included in the models of Asano et al. ${ }^{143}$ and Itoh $e t$ al. ${ }^{144}$ by introducing the spin-dependent multilayer potential and in the model of Todorov $e$ al. ${ }^{146}$ by using the spin-dependent band widths, in general single-band tight-binding models are not able to describe accurately the spin-polarized band structure of real magnetic layered systems. A more accurate band structure was considered by Itoh et al. ${ }^{147}$ for studying the material dependence of magnetoresistance and thermoelectric power in $\mathrm{Fe} / \mathrm{TM}$ and $\mathrm{Co} / \mathrm{TM}$ multilayers, where $\mathrm{TM}=\mathrm{Sc}$, Ti, $\mathrm{V}, \mathrm{Cr}, \mathrm{Mn}, \mathrm{Ru}, \mathrm{Rh}$, or Pd. They used the tight-binding $d$-band model and calculated self-consistently the electronic structure and the magnetic moments of rough $\mathrm{Fe} / \mathrm{TM}$ and $\mathrm{Co} / \mathrm{TM}$ interfaces. They ignored, however, the contribution from the $s p$ bands and their hybridization with the $d$ bands which is, as we will see below, crucial for GMR.

\section{MULTIBAND MODELS}

As has already been noted above, free-electron and single-band $s$-valent tight-binding models oversimplify the electronic structure of the magnetic multilayers which are used in GMR experiments These models cannot account realistically for the angular character of the $d$ orbitals, which is partly responsible for the magnetism in transition metal ferromagnets. They neglect, moreover, the contribution from the $d$ bands to the electronic transport, which is important due to the presence of the $d$ bands at the Fermi energy, as for the minority-spin electrons in Co (see Fig.3c). These models also ignore the hybridization between the $s p$ and $d$ electrons which is, as we will see, very important for GMR. Only accurate multiband models can adequately take into account the above effects.

Powerful methods of electronic band structure calculations have been developed during the last few decades, which are based on density-functional theory (for a review see, e.g., ref.148). These methods do not involve any empirical parameters and are therefore referred to as "ab-initio" or "firs principles". Density-functional theory is a general approach for calculating the ground-state propertie of an interacting electron gas in the presence of an external potential. It is based on the Hohenberg which states that the ground-state energy of the many body system is a unique functional of the electronic charge density $n(\mathbf{r})$. Kohn and Sham ${ }^{150}$ showed that minimizing the total energy under assumption of the electronic charge density being a sum over all occupied single-particle states $\psi_{i}(\mathbf{r})$, i.e.

$$
n(\mathbf{r})=\sum_{i \in o c c}\left|\psi_{i}(\mathbf{r})\right|^{2},
$$

leads to the set of self-consistent equations

$$
\left[-\frac{\hbar^{2}}{2 m} \nabla^{2}+V_{e x t}(\mathbf{r})+e^{2} \int d \mathbf{r}^{\prime} \frac{n\left(\mathbf{r}^{\prime}\right)}{\left|\mathbf{r}-\mathbf{r}^{\prime}\right|}+\frac{\delta E_{x c}[n(\mathbf{r})]}{\delta n(\mathbf{r})}\right] \psi_{i}(\mathbf{r})=E_{i} \psi_{i}(\mathbf{r}) .
$$

Here the wave functions $\psi_{i}(\mathbf{r})$ and eigen-energies $E_{i}$ are labeled according to a state index $i$. Equation (V.2) is similar to a single-particle Schrödinger equation with an effective Hamiltonian, in which the first term is the kinetic energy, the second term is the external potential that includes the electrostatic potential from the nuclei, the third term is the Hartree potential of the total electronic charge distribution, and the fourth term is the exchange-correlation potential. The exchange-correlation potential includes all many-body effects and, therefore, cannot be calculated exactly. The common approximation is the local density approximation (LDA). Within the LDA the exchange-correlation energy $E_{x c}[n(\mathbf{r})]$ for an inhomogeneous system is assumed to be given by the exchange-correlation energy density $\epsilon_{x c}[n(\mathbf{r})]$ for a homogeneous electron gas of charge density $n(\mathbf{r})$ so that $E_{x c}[n(\mathbf{r})]=\int \epsilon_{x c}[n(\mathbf{r})] n(\mathbf{r}) d \mathbf{r}$. The LDA is formally justified for slowly varying densities, but it has been found in practice to provide a good description of the ground state properties of non-magnetic transition metals, in addition to the more free-electron-like $s p$-valent metals. In particular, density functional formalism can be extended to magnetic systems ${ }^{151}$ by introducing the spin densities $n^{\uparrow}(\mathbf{r})$ and $n^{\downarrow}(\mathbf{r})$, so that the charge density is $n(\mathbf{r})=n^{\uparrow}(\mathbf{r})+n^{\downarrow}(\mathbf{r})$. In this case the single-particle wave functions become spin-dependent, the exchange-correlation energy becomes a functional of the spin densities, $E_{x c}\left[n^{\uparrow}(\mathbf{r}), n^{\downarrow}(\mathbf{r})\right]$, and the LDA is generalized by the LSDA, which is the local spin-density approximation. ${ }^{152}$ The Kohn-Sham equations (V.2) have to be solved self-consistently, because both the Hartree and exchange-correlation potentials depend on the output density. The resulting singleparticle eigenfunctions $\psi_{i}(\mathbf{r})$ and eigenvalues $E_{i}$ can be used for calculating transport properties. ${ }^{153}$

A variety of techniques have been developed to solve the Kohn-Sham equations, among them the augmented plane wave method (APW) ${ }^{154}$ the Green's function Korringa-Kohn-Rostoker (KKR) method $^{155}$ and the linear-muffin-tin orbital method (LMTO). ${ }^{156}$ All these methods can be method $^{155}$ and the linear-muffin-tin orbital method (LMTO). ${ }^{156}$ All these methods can be
implemented within the "full-potential" approach, which provides a numerically exact solution. Most of calculations are, however, performed using shape approximations for the potential, such as the muffin-tin approximation or the atomic sphere approximation (ASA), which work well for closepacked crystal structures. These approximations make the computations much faster without significant loss in accuracy. If applied to the electronic band structure of the bulk transition $3 d$ metal all these methods give similar results. An example of the band structure calculated using the LMTOASA method is given in Fig. 5 for bulk $\mathrm{Cu}$ and $\mathrm{Co}$.

The multiband tight-binding approximation is a more empirical approach for calculating the electronic band structure, which is a generalization of the single-band approximation considered in section 14. Although not as accurate as the ab-initio models, the multiband tight-binding models provide valuable insight into electronic transport by simplifying the physics involved. They also allow considerable saving in computer time compared to full-scale first-principles calculations. The multiband models for transition metals include the valence $s, p$ and $d$ orbitals, which are characterized by different on-site atomic energy levels. The effects of bonding and hybridization enter through twocenter hopping integrals between atomic orbitals with characteristic angular momenta. The angular variation in the hopping matrix elements with neighboring sites has been tabulated by Slater and Koster. ${ }^{157}$ Spin polarization is treated by introducing two different sets of tight-binding parameters for the majority and minority spins. For the $3 d$ ferromagnets sizable spin dependence appears, however, only for the on-site energies of the $d$ orbitals, which reflects the Stoner exchange spitting of the spin bands. ${ }^{34}$ The values of the tight-binding parameters, i.e. the hopping integrals and the on-site atomic energies, can be obtained by fitting the tight-binding bands to the bands calculated by the firstprinciple methods described above. ${ }^{158}$ This makes it possible to represent accurately the electronic structure of the transition metals. The electronic structure for a multilayer can be built either by fitting the ab-initio band structure for this multilayer or from the tight-binding parameters of the respective bulk metals. In the latter case the hopping integrals between non-equivalent species can be set as the geometric mean of the respective hopping integrals for each species and the on-site energies can be set to equate the Fermi energies of the respective bulk metals. In this approximation the tight-binding model for the multilayer does not describe self-consistently the rearrangement of the electronic charge at the interfaces. Some self-consistency can be introduced using the condition of local charge 
neutrality, ${ }^{159}$ in which the relative position of the on-site atomic energies is adjusted to guarantee zero net charge on each atom. This is a reasonable approximation for metallic multilayers.

The multiband tight-binding model can be derived from first-principles using the tight-binding linear muffin-tin orbital (TB-LMTO) method, ${ }^{160}$ in which the basis muffin-tin orbitals are screened by the presence of their surrounding neighbors into a more localized or tight-binding form. Using the tabulated LMTO structure constants a set of nearest-neighbor tight-binding parameters can be generated for a magnetic layered system by solving the Dyson equation for the screening selfconsistently. These parameters can then be used as input for calculating conductance.

The above first-principle and tight-binding methods can be used for calculating the electronic structure of magnetic thin-film systems. However, in order to analyze transport properties, these models have to be extended to include disorder, which generates a random potential and scatters the electrons at the Fermi energy. Including disorder is important because all the experiments on GMR performed to date are carried out in the diffusive regime, in which the conductance is limited by scattering. Within the diffusive regime the sample dimensions are much larger than the mean free path. A realistic description of disorder is one of the key problems for predicting GMR because depending on the model for disorder the result for GMR can be very different. We will discus various approached to include disorder and to treat GMR in the diffusive regime of conduction in sections 16-18.

Opposite to the diffusive transport regime, the ballistic regime assumes that the sample dimensions are much smaller than the mean free path. ${ }^{161}$ In this regime the conductance is not affected by scattering but is determined entirely by the band structure and the device geometry. All the complications resulting from the disorder in the diffusive regime do not exist in the ballistic regime, which allows parameter-free first-principle calculations of GMR to be performed. ${ }^{162,163,22}$ Although the ballistic regime has not yet been probed experimentally in GMR materials, considering this limit for conduction is useful to demonstrate the importance of the spin-polarized band structure for GMR. This point will be illustrated in the next section.

\section{Ballistic limit}

The conductance of a disorder-free system is determined by the kinematic motion of the electrons. Even though the electrons passing through the sample are not scattered, the conductance of the sample is finite due to its finite cross-section. The magnitude of the conductance is determined by the electronic band structure of the material from which the sample is fabricated and can be calculated the electronic band structure of the material from which the sample is fabricated and can be calculated
from first principles. In order to derive the expression for the ballistic conductance $\Gamma$ we write down, first, the expression for the net current which flows across the sample of cross section $A$ due to applied voltage $V$. The net current per spin, $I$, is given by the difference in the number of electron propagating in opposite directions, i.e.

$$
I=\int_{A} d \mathbf{s} \cdot \sum_{\eta} \int \frac{d \mathbf{k}}{(2 \pi)^{3}} e \mathbf{v}_{\eta}(\mathbf{k})\left\{f\left[E_{\eta}(\mathbf{k})-e V\right]-f\left[E_{\eta}(\mathbf{k})\right]\right\},
$$

where $f(E)$ is the Fermi distribution function, $d \mathbf{s}$ is a cross sectional element of the sample, and the velocity of band $\eta$ can be determined from the band dispersion $E_{\eta}(\mathbf{k})$ by $\mathbf{v}_{\eta}(\mathbf{k})=d E_{\eta}(\mathbf{k}) / \hbar d \mathbf{k}$ Here as before we have omitted the spin indices. Assuming that the voltage is small and taking the zero-temperature limit we arrive at the conductance

$$
\Gamma=\left(\frac{I}{V}\right)_{V \rightarrow 0}=A e^{2} \sum_{\eta} \int \frac{d \mathbf{k}}{(2 \pi)^{3}} \frac{1}{2}\left|\mathbf{n} \cdot \mathbf{v}_{\eta}(\mathbf{k})\right| \delta\left[E_{\eta}(\mathbf{k})-E_{F}\right],
$$

where $\mathbf{n}$ is a unit vector in the transport direction and the factor $1 / 2$ appears because only electron moving in one direction contribute to the current. Mathematically this formula represents a weighted density of states at the Fermi energy. This formula is suitable for practical calculations of the ballistic conductance and can be applied to magnetic multilayers

The formula (15.2) can be rewritten in a different way. Performing explicitly the integration in equation (15.2) with respect to the momentum $\mathrm{k}_{z}$ along the direction of transport $z$ we obtain

$$
\Gamma=\frac{e^{2}}{\pi \hbar} \frac{A}{4 \pi^{2}} \sum_{\eta} \int d \mathbf{k}_{\|} Q_{\eta}\left(\mathbf{k}_{\|}\right)=\frac{e^{2}}{\pi \hbar} N,
$$

where $\mathbf{k}_{\|}$is the transverse momentum, i.e. the momentum perpendicular to the current, and $Q_{\eta}\left(\mathbf{k}_{\|}\right)$is the number of roots in the equation $E_{\eta}\left(\mathbf{k}_{\|}, \mathrm{k}_{z}\right)=E_{F}$ for a given $\mathbf{k}_{\|}$and spin. This representation is equivalent to the Landauer expression for the ballistic conductance (see, e.g., ref. 22 for a discussion). According to equation (15.3) the conductance is determined by the number of conducting channels $N$ opened for the current-carrying electrons, which is determined by the density of the transverse modes at the Fermi energy.

We note that the ballistic conductance of an ideal periodic structure does not depend on the length $L$ of the sample along the direction of the current (which makes the conductivity infinite). A long as $L$ is much smaller than the mean free path, the conductance per unit area is determined only by the number of open conducting channels, i.e. entirely by the band structure. We also note that unlike conductivity, the ballistic conductance in systems with cubic symmetry is, in general, not isotropic.
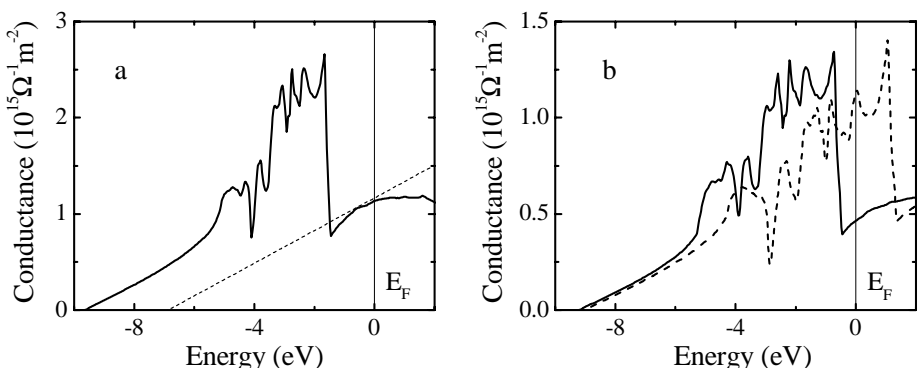

Fig.27 Ballistic conductance in the (001) direction for bulk $f c c \mathrm{Cu}$ (a) and bulk $f c c$ Co (b) as a function of electron energy within a rigid band structure. The vertical line displays the Fermi energy. The dashed line in figure (a) shows the free-electron result. The solid and dashed lines in figure (b) show the conductance of the majority and minority spins respectively. After Schep et al. ${ }^{163}$

Using the LMTO-ASA method and formula (15.2) Schep et al. ${ }^{163}$ calculated the ballistic conductance of bulk $\mathrm{Cu}$ and Co metals, which is shown in Figs.27a,b respectively. Although only the value at the Fermi energy is relevant, the conductance is plotted as a function of the band filling to indicate the contributions from different bands. Schep et al. found that at the Fermi energy the ballistic conductance of $\mathrm{Cu}$ is well described by the free-electron estimate (see the dashed line in Fig.27a). For the energy range between 1.5 and $4 \mathrm{eV}$ below the Fermi energy the ballistic conductance is significantly enhanced due to the high density of electrons of mainly $d$ character. This is opposite to the diffusive regime in which the heavy $d$ electrons contribute little to the conductance (see the 
discussion in section 2). The enhancement is, however, considerably less pronounced than that in the corresponding density of states (compare to Fig.5a). This is due to the weighting in equation (15.2) with the velocity, the latter being much smaller for the relatively flat bands in this energy range. Fo the energies more than $6 \mathrm{eV}$ below the Fermi energy there is only one band which has mainly character. In magnetic Co the degeneracy between the up- and down-spin electrons is lifted. As is seen from Fig.27b, the ballistic conductance depends on the spin direction due to the spin dependence of the electronic structure. For the majority-spin electrons the ballistic conductance resembles that in $\mathrm{Cu}$. The minority-spin $d$ bands are shifted to higher energies resulting in the higher minority-spin conductance at the Fermi energy. In contrast to the results for $\mathrm{Cu}$ and for the majority spins of $\mathrm{Co}$, Schep et al. found no reasonable free-electron estimate for the minority-spin conductance of $\mathrm{Co}$, which reflects the complicated band structure of Co for this spin direction at the Fermi energy.

The difference in the electronic structure for the majority and minority spins in bulk Co at the Fermi energy leads to a difference in the spin conductance, which inevitably should affect the conductance of a $\mathrm{Co} / \mathrm{Cu}$ multilayer and hence result in GMR. This has, indeed, been found by Schep et al. ${ }^{162}$ who predicted that in the ballistic regime the CPP conductance increases by more than factor of two when the relative orientation of the magnetizations of adjacent magnetic layers in $\mathrm{Co} / \mathrm{Cu}$ multilayer is changed from antiparallel (AP) to parallel (P). Such a sizable difference in the conductance reflects the contribution from electrons of different orbital momenta to the current According to equation (15.3) the ballistic conductance is determined by the geometric properties of the Fermi surface, namely the projection of the Fermi surface in the direction of the current $Q_{\eta}\left(\mathbf{k}_{\|}\right)$. Schep et al. investigated this projection in $\mathbf{k}_{\|}$space for the $\mathrm{Co} / \mathrm{Cu}$ multilayers and found a significant difference between the majority and minority spins. Although for the majority spins within the $\mathrm{P}$ configuration $Q_{\eta}\left(\mathbf{k}_{\|}\right)$resembles the free-electron picture, the minority spins within the $\mathrm{P}$ configuration and both spins within the AP configuration are not free-electron-like. The majority-spin $s$ electrons shunt the current within the $\mathrm{P}$ magnetizations making the conductance for this configuration much higher than that for the AP configuration, which is mainly determined by the $d$ electrons.

Fig.28 shows the variation of the ballistic conductance and GMR for $\mathrm{Co}_{\mathrm{n}} / \mathrm{Cu}_{\mathrm{n}}$ multilayers as a function of the layer thickness $n$ for the CPP and CIP geometries. ${ }^{163}$ We see from Fig.28a that for small layer thicknesses the CPP conductance decreases rapidly with $n$, which arises from the evanescent states in the $\mathrm{Co}$ and $\mathrm{Cu}$ layers and from the deviations in the potential near the interfaces corresponding to CPP GMR of about 100\%. We see from Fig.28b that the GMR in the CIP geometry is much lower than in the CPP geometry and decreases with the layer thickness. In this case the minority-spin electrons display a higher conductance within the $\mathrm{P}$ configuration reflecting that in bulk Co. We note that the calculated values of CIP GMR are much lower than the experimental value obtained for the $\mathrm{Co} / \mathrm{Cu}$ multilayers (e.g., ref.44). This indicates that scattering must be included in order to obtain agreement with experiments.

As is seen from Fig.28, both the CIP and CPP conductances display small damped oscillations as a function of the number of monolayers. These oscillations originate from quantum size effects an were first predicted by Mathon et al. ${ }^{164}$ for the CPP GMR within the ballistic transport regime. Using a single-band tight-binding model and the recursive approach Mathon $e t$ al. predicted the presence of two types of oscillation. The first type of oscillation results from stationary points at the Fermi surface and has a period, which is determined by the wave vector spanning the Fermi surface of the spacer layer in the direction perpendicular to the planes. This type of oscillation has the same period as that layer in the direction perpendicular to the planes. This type of oscillation has the same period as that
predicted earlier for the oscillatory exchange coupling. ${ }^{165}$ The second type of oscillation results from potential steps at the interfaces and is determined by the transmission cutoffs of the transmitting state close to specular reflection. Using a similar approach to calculate the ballistic conductance and CP GMR in $\mathrm{Co} / \mathrm{Cu}$ multilayers within a multiband tight-binding model Mathon et al. ${ }^{166}$ found the presence of both types of oscillation periods. They showed that the conductance oscillations for the majority-spin electrons within the P configuration have periods, which are determined by the stationary points at the Fermi surface of $\mathrm{Cu}$. On the other hand, the conductance oscillations of the minority-spin electrons in the P configuration and either-spin electrons in the AP configurations are dominated by the periods which are determined by the conductance cutoff due to a mismatch between the $\mathrm{Co}$ and $\mathrm{Cu}$ bands across the $\mathrm{Co} / \mathrm{Cu}$ interfaces. We note that neither type of oscillation has yet been observed in experiments on CPP transport, which is most probably due to the interface and bulk disorder in real multilayers. The latter fact is confirmed by calculations in the diffusive regime of conduction, which will be presented in section VI.

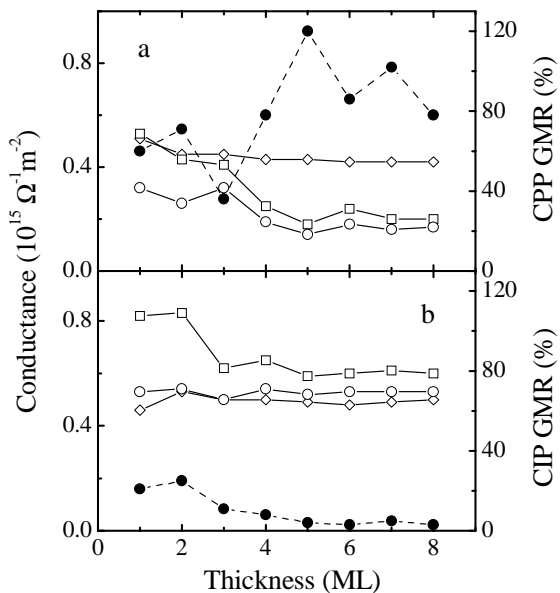

Fig.28 Dependence of GMR (solid circles) and conductance of the (001)-oriented $\mathrm{Co}_{\mathrm{n}} / \mathrm{Cu}$ multilayers for the majority-spin (diamonds) and the minority-spin (squares) electrons in (open circles) within $\mathrm{CPP}$ (a) and CIP (b) geometries. After Schep et al ${ }^{16}$

Schep et al. ${ }^{162}$ have demonstrated the crucial role of the hybridization between the dispersive $s p$ bands and the localized $d$ bands for GMR within the ballistic regime. They switched off the $s p-d$ hybridization by setting the matrix elements between the $s p$ and $d$ orbitals in the LMTO structure constants equal to zero. This resulted in a significant drop of CPP GMR, e.g., from $120 \%$ to $3 \%$ for the (001)-oriented $\mathrm{Co}_{5} / \mathrm{Cu}_{5}$ multilayer. The origin of this effect can be explained as follows. The unhybridized $s p$ and $d$ bands carry the electric current independently and can thus be considered as two parallel conduction channels. In the absence of hybridization the $s p$ electrons are not spin polarized and, therefore, do not contribute to GMR. On the other hand, although the $d$ electrons in Co are spin-polarized, they do not contribute to the conductance because in the absence of the $s p-d$ hybridization they are not coupled to the $s p$ bands of $\mathrm{Cu}$ (which are the only bands at the Fermi level) and, therefore, can not be transmitted across the spacer layer. The importance of the $s p-d$ hybridization for GMR suggests that theories which ignore this hybridization are leaving out a crucia ingredient. We will see in section 17 that the $s p-d$ hybridization also plays a decisive role for GMR within the diffusive regime. 


\section{Semiclassical theor}

Accurate models for the band structure can be incorporated within semiclassical transport theory to calculate GMR in magnetic multilayers in the diffusive regime of conduction. The simplest way to proceed is to use the relaxation time approximation, as was described in section 12 . In this case the conductivity of a bulk homogeneous system can be calculated using expression (12.10). Although magnetic multilayers are not homogeneous in the direction perpendicular to the planes, one can still use this expression within the CIP geometry, because as was discussed in section 13 the internal electric field is a constant in this case due to the homogeneity of the multilayers in the plane of the layers. The validity of the relaxation time approximation is often not known, but even within thi approximation an accurate quantum-mechanical calculation of the relaxation time is not trivial and, as we will see below, requires sophisticated techniques. In addition, the relaxation time depends on the model for disorder which determines the mechanism of scattering.

The simplest approach is to assume a constant relaxation time, as was done by Oguchi. ${ }^{167}$ In this case formula (12.10) for the conductivity in the $v$ direction per spin can be rewritten as

$$
\sigma^{v v}=e^{2} \tau \sum_{\eta} \int \frac{\mathrm{d} \mathbf{k}}{(2 \pi)^{3}}\left|\mathrm{v}_{\eta}^{v}(\mathbf{k})\right|^{2} \delta\left[E_{\eta}(\mathbf{k})-E_{F}\right]
$$

where we have introduced the integration over the Brillouin zone and displayed explicitly the summation over band index $\eta$. As is seen, the conductivity is factorised into a scattering term $\tau$ and the electronic structure term. The electronic structure term is similar to that in the ballistic regime of conduction (compare to equation (15.2)) and can be evaluated without any free parameters. Within the approximation of a state- and spin-independent relaxation time $\tau$ the GMR ratio does not depend on $\tau$ and is entirely determined by the band structure. Oguchi calculated self-consistently the electronic band structure of $\mathrm{a} \mathrm{Co}_{3} / \mathrm{Cu}_{3}$ multilayer using the LMTO method and found values of GMR of $47 \%$ fo CIP and $170 \%$ for CPP. These values are higher than those obtained within the ballistic regime (although of the same order of magnitude), which is due to the additional velocity-weighting factor in formula (16.1) which enhances the spin asymmetry. Oguchi was the first to point out the importance of the band structure (the Fermi velocities) for GMR. However, his assumption of a state- and spinindependent relaxation time is difficult to justify. In particular, neglecting the spin dependence of the $\tau$ is unrealistic, at least due to the density of states factor in equation (1.4) which is spin-dependent.

Zahn et al. ${ }^{168}$ used expression (16.1) for the spin conductivity to calculate GMR in $\mathrm{Fe} / \mathrm{C}$ multilayers, assuming that the relaxation time is spin-dependent (but still state-independent). Scattering was introduced into their model by assuming the presence of $\mathrm{Cr}$ impurities in the $\mathrm{Fe}$ layer within the low-concentration limit. They used an explicit expression for the spin-dependent scattering probability

$$
P_{\eta \mathbf{k}, \eta^{\prime} \mathbf{k}^{\prime}}=\frac{2 \pi}{\hbar}\left|T_{\eta \mathbf{k}, \eta^{\prime} \mathbf{k}^{\prime}}\right|^{2} \delta\left(E_{\eta \mathbf{k}}-E_{\eta \mathbf{k}^{\prime}}\right),
$$

in which the $T$ matrix describes the scattering of an electron by an impurity embedded in the multilayer. This expression can be considered as a generalization of the Fermi golden rule (1.4) to include all orders of perturbation theory with respect to the scattering potential. The matrix elements $T_{\eta \mathbf{k}, \eta \mathbf{k}^{\prime}}$ were calculated using an application of multiple-scattering theory within the first-principle KKR Green's function formalism, which was developed earlier in works by Dederichs and coauthors. ${ }^{169}$ Using these matrix elements obtained for a Cr impurity in bulk Fe, for either spin direction the state-independent relaxation time was calculated from expression (16.2) by a summation ove state indices according to

$$
\tau_{\eta}^{-1}(\mathbf{k})=\sum_{\eta^{\prime} \mathbf{k}^{\prime}} P_{\eta \mathbf{k}, \eta^{\prime} \mathbf{k}^{\prime}}
$$

and then by averaging over the Fermi surface. It was found that the ratio of the spin-relaxation time $\alpha=\tau^{\uparrow} / \tau_{\downarrow}=0.11$, which implies that the majority spins are scattered strongly at a $\mathrm{Cr}$ defect, whereas the minority spins pass the defect weakly scattered. The relaxation time for the antiparalle configuration was estimated by a series-resistor rule, i.e. $1 / \tau_{A P}=\left(1 / \tau^{\uparrow}+1 / \tau_{\downarrow}\right) / 2$. Zahn et al. calculated CIP and CPP magnetoresistance within an optimised LCAO (linear combination of atomic orbitals) method using larger unit cells $\left(\mathrm{Fe}_{3} / \mathrm{Cr}_{n}\right.$ and $\mathrm{Fe}_{m} / \mathrm{Cr}_{4}$ with $\left.n, m \leq 12\right)$ than those which were used by Oguchi. ${ }^{167}$ They predicted really giant effects of about $600 \%$ for CIP and of about $2500 \%$ for $\mathrm{CPP}$, which are much higher than the experimental values for $\mathrm{Fe} / \mathrm{Cr}$ multilayers.

The approximation of a state-independent relaxation time was lifted in the work by Binder $e$ $a l .{ }^{170}$ of the same group, who calculated the state-dependent relaxation times and the spin-dependen conductivity of the $\mathrm{Co} / \mathrm{Cu}$ multilayer within a screened KKR method using semiclassical transpor theory. They introduced different $3 d$-metal impurities at the Co interface layer and compared the resulting resistivities and GMR for the state-dependent and state-independent relaxation times. Thei results show that the approximation of a state-independent relaxation time overestimates the resistivity of the majority-spin electrons by a factor of two and of the minority-spin electrons by about $10 \%$, which leads to overestimating GMR. Nevertheless, the predicted values of GMR for the Fe, N and $\mathrm{Cu}$ impurities are still huge (about $4000 \%$ for $\mathrm{Fe}$ and $6000 \%$ for $\mathrm{Ni}$ ). For $\mathrm{Ti}, \mathrm{V}$ and $\mathrm{Cr}$ impurities an inverse GMR effect was obtained. Binder $e t$ al. also investigated the validity of the relaxation-time approximation. They extended their semiclassical theory to calculate the scattering-in term (see section 12) by solving the Boltzmann equation iteratively. They found that the relaxation time approximation gives very satisfactory results for the systems considered.

Butler et al. ${ }^{171}$ used a different model for disorder to calculate CPP GMR in the (111)-oriented $\mathrm{Co}_{\mathrm{n}} / \mathrm{Cu}_{\mathrm{m}}$ multilayers (up to $\mathrm{n}=\mathrm{m}=6$ ) within the semiclassical approximation. They assumed that the $\mathrm{Co} / \mathrm{Cu}$ interfaces are interdiffused with $1 \%$ intermixing of the $\mathrm{Co}$ and $\mathrm{Cu}$ atoms. The band structure of the disordered multilayers was calculated using the single-site coherent potential approximation within the KKR Green's function method. Assuming a sufficiently weak impurity scattering they computed the relaxation time for each state at the Fermi energy, which is inversely proportional to the imaginary part of the self-energy in equation (13.12). Then, using the state-dependent relaxation time they solved the Boltzmann equation disregarding the scattering-in term and calculated the for the $\mathrm{Co} / \mathrm{Cu}$ multilayers were huge varying from about $1000 \%$ to $2000 \%$ for different layer thicknesses. Similar calculations performed by Butler et al. ${ }^{171}$ for the (111)-oriented $\mathrm{Fe}_{20} \mathrm{Ni}_{80} / \mathrm{Cu}$ multilayers found even larger GMR values.

Nesbet ${ }^{172}$ reached a similar conclusion for $\mathrm{GMR}$ in $\mathrm{Co} / \mathrm{Cu}_{2}$ and $\mathrm{Fe} / \mathrm{Cr}_{2}$ multilayers. He calculated the state-dependent relaxation time according to equations (16.2) and (16.3) assuming that scattering is caused by interdiffused atoms at the interfaces. The spin-polarized band structure and the scattering $T$-matrix were computed by expanding the Bloch wave-functions into a large number of atomic orbitals, using a full-potential multiple-scattering approach within the local density approximation The semiclassical expression for conductivity within the relaxation time approximation (12.10) was used to compute GMR. Consistent with previous calculations magnetoresistance due to this scattering mechanism was found to be very large.

The highly overestimated values of GMR obtained by Zahn et al. ${ }^{168}$ Binder et al..${ }^{170}$ Butler $e$ $a l .,{ }^{171}$ and Nesbet ${ }^{172}$ is a direct consequence of the fact that the electronic structure of the impurities is nearly identical to the electronic structure of the host atoms in one of the two spin channels which leads to no scattering and shunting within this spin channel. This can be illustrated by calculations of the number of valence electrons per atom in the $\mathrm{Co} / \mathrm{Cu}$ and $\mathrm{Fe} / \mathrm{Cr}$ systems shown in Figs.29a,b. ${ }^{173}$ As is seen from Fig.29a, in the $\mathrm{Co} / \mathrm{Cu}$ system the number of $\mathrm{Co}$ majority-spin electrons matches very closely the number of $\mathrm{Cu}$ electrons, whereas there is a strong mismatch in the number of minority- 
spin electrons. This matching in the majority-spin channel implies that the atomic cobalt and copper potentials appear very similar to the majority-spin electrons leading to no scattering within this spin channel when $\mathrm{Cu}$ impurities are imbedded in Co or vice versa. The same conclusion was obtained for a $\mathrm{Ni}_{80} \mathrm{Fe}_{20} / \mathrm{Cu}$ multilayer because the Fermi energy scattering amplitudes for majority-spin cobalt, majority-spin nickel, and majority-spin iron (as an impurity in nickel) are all very similar. ${ }^{173}$ For the $\mathrm{Fe} / \mathrm{Cr}$ system the matching in the number of valence electrons per atom occurs for the minority spins $\mathrm{Fe} / \mathrm{Cr}$ system the matching in the number of valence electrons per atom occurs for
leading to a weak scattering of electrons in the minority-spin channel (see Fig.29b)

As was suggested by Butler et al. ${ }^{173}$ the spin-orbit interaction, which was not considered in the above calculations, might partly explain the discrepancy between theory and experiment. Indeed, in bulk alloys the spin-orbit interaction, although small, is sufficient to avoid the near short circuits due to lack of scattering in either spin channel (e.g., ref.174). Another reason could be the presence of misaligned magnetic moments at rough interfaces due to the reduced exchange coupling, which as we have seen in section 8 might play a role. However, we believe that the principle disagreement between the theory and experiment originates from the neglect of scattering by intrinsic defects, which are always present in the multilayer. In the above calculations it is assumed that the introduced impuritie are the only source of scattering, which is not the case in real experiments on GMR. We will see in section 17 that a realistic model for disorder accounts for the experimentally-observed values of GMR in the $\mathrm{Co} / \mathrm{Cu}$ and $\mathrm{Fe} / \mathrm{Cr}$ multilayers.

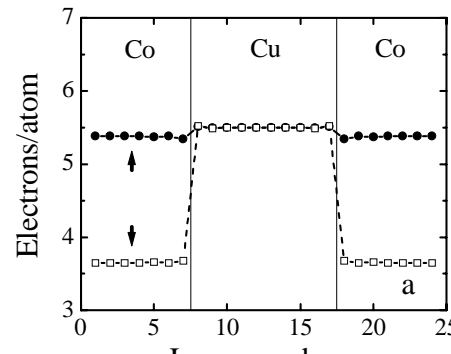

Layer number

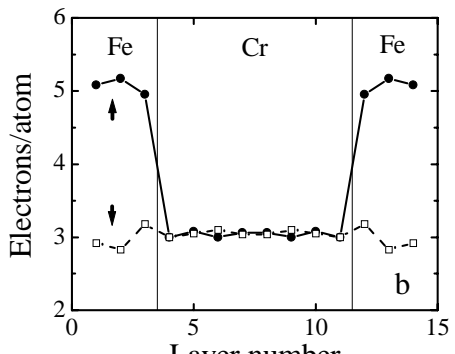

Layer number
Fig.29 Calculated number of valence electrons for the (111)-oriented $\mathrm{Co} / \mathrm{Cu} / \mathrm{Co}$ (a) and (001)oriented $\mathrm{Fe} / \mathrm{Cr} / \mathrm{Fe}$ (b) trilayers for the majority-spin ( $\uparrow$ ) and minority-spin $(\downarrow)$ electrons. Note the matching for the majority spins in the $\mathrm{Co} / \mathrm{Cu} / \mathrm{Co}$ and matching for the minority spins in the $\mathrm{Fe} / \mathrm{Cr} / \mathrm{Fe}$. After Butler et al. ${ }^{173}$

An interesting calculation was performed by Zahn et al.,${ }^{175}$ who investigated the effect of the impurity position on GMR in $\mathrm{Co} / \mathrm{Cu}$ multilayers. In order to study impurities with different scattering spin asymmetries they assumed that the spin-dependent scattering potential $V$ is a parameter, thereby neglecting details of the impurity potential and its dependence on the site position $\mathbf{r}_{i}$ within the supercell. The scattering spin asymmetry was, therefore, defined as $\alpha=V^{\uparrow} / V_{\downarrow}$. They considered the weak scattering limit in which the scattering rate (the inverse relaxation time) is determined by the Born approximation

$$
\tau_{\eta}^{-1}\left(\mathbf{k}, \mathbf{r}_{i}\right)=\frac{2 \pi}{\hbar} V^{2}\left|\psi_{\eta}\left(\mathbf{k}, \mathbf{r}_{i}\right)\right|^{2} n\left(\mathbf{r}_{i}, E_{F}\right)+\tau_{0}^{-1},
$$

where $\psi_{\eta}\left(\mathbf{k}, \mathbf{r}_{i}\right)$ is the Bloch wave function of the multilayer without the impurities and $n\left(\mathbf{r}_{i}, E_{F}\right)$ is the local density of states. In order to avoid short circuit effects due to states with low scattering amplitude at the impurity position they added a constant scattering rate $\tau_{0}^{-1}$ in equation (16.4). Since the scattering rate in equation (16.4) is proportional to the spin-dependent density of states at the impurity site $n\left(\mathbf{r}_{i}, E_{F}\right)$, a strong position dependence of GMR is expected. Zahn et al. found that $n\left(\mathbf{r}_{i}, E_{F}\right)$ is enhanced and have a strongest spin asymmetry at the Co interface in a $\mathrm{Co}_{9} / \mathrm{Co}_{7}$ mutlilayer, which is explained by the formation of quantum-well and interface states. Due to this the magnitude of GMR is enhanced by the impurities with no spin asymmetry $(\alpha=1)$ when they are placed at the $\mathrm{Co}$ interface. This is evident from the triangles in Fig.30, which shows the calculated values of GMR for various positions and scattering spin asymmetries of the impurities. For impurities with a stronger scattering of the minority than the majority electrons, $\alpha>1$, the existing spin asymmetry in the local density of states is amplified and leads to an even stronger enhancement of GMR (the circles in Fig.30). For the opposite spin asymmetry, $\alpha<1$, the spin asymmetry of the local DOS and the scattering potential compensate each other and GMR is reduced. As is seen from Fig.30, impuritie placed within the $\mathrm{Cu}$ layer are ineffective for GMR, which is ascribed to the small local DOS in $\mathrm{Cu}$.

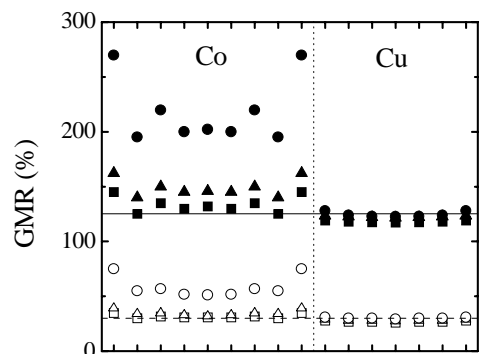

Impurity position (ML)

Fig.30 Calculated CIP (opened symbols) and CPP GMR (closed symbols) of the (001)-oriented $\mathrm{Co}_{9} / \mathrm{Cu}_{7}$ multilayer as a function of the impurity position within the multilayer for the impurities with various asymmetries in the scattering potential $\alpha=V^{\uparrow} / V_{\downarrow}: \alpha=1$ (triangles), $\alpha=4$ (circles), and $\alpha=0.25$ (squares). The solid and dashed lines show the values of CPP and CIP GMR respectively calculated for the scattering rate $\tau_{0}^{-1}$ ( $V=0$ in equation (16.3)). The vertical dotted line separates the Co and Cu layers. After Zahn et al. ${ }^{175}$

It is interesting to compare the predictions of Zahn et al. ${ }^{175}$ with the results of experiments by Marrows and Hickey, ${ }^{76}$ (see section 8). Experimentally it was found that impurities with $\alpha<1$ suppress GMR, usually to a great extent when they are placed at the interface, and still have a considerable effect when they lie several lattice constants away from the interface; impurities with $\alpha>1$ sometimes do provide an enhancement of GMR, but it is only to be found when they are a few ML behind the $\mathrm{Co} / \mathrm{Cu}$ interface; and impurities in the spacer layer have a dramatic effect by lowering GMR. These findings are at odds with the theoretical predictions of Zahn et al. Marrows and Hickey ${ }^{76}$ suggested that to reconcile the data with the theory one must admit in addition to the scattering by impuritie considerable spin-dependent scattering in the bulk of the Co layers, or that the impurities can affect 
the local density of states up to several atomic sites away. Very recent calculations performed by the same group ${ }^{176}$ demonstrate, however, better agreement between the theory and the experiment.

A necessary condition for the validity of the semiclassical Boltzmann theory is a sufficiently low impurity/defect density. In layered systems the Boltzmann formalism breaks down when the subban energy splitting becomes comparable to the life-time broadening $\hbar / 2 \tau$ due to scattering by impurities and/or defects. As we will see in the next section, this is the case for real multilayer structures, which are used in experiments on GMR. The quantum-mechanical treatment of transport is necessary in order to make a quantitative comparison between theory and experiment.

\section{Tight-binding models}

Experimental data show that magnetic layered systems contain a lot of intrinsic structural defects such as vacancies, stacking faults, lattice distortions, misfit dislocations and grain boundaries that are produced during the process of deposition (e.g., ref.177,178). These defects influence the residual resistivity making it much larger in thin films than in corresponding bulk metals. For example, Rijk et al. ${ }^{178}$ found significant scattering at the grain boundaries and other defects in sputter-deposited copper and permalloy thin films which was dependent on the layer thickness. Scattering by these defects must, therefore, be included in a realistic model for GMR. However, simultaneous modeling of all these defects including their structural and electronic properties is very complicated and of all these defects including their structural and electronic properties is very complicated and
probably pointless because we do not have sufficient experimental information about distribution, concentration, strength and other parameters characterizing these defects. A simplified approach to include defect scattering in a model for GMR was developed by Tsymbal and Pettifor ${ }^{179}$ within a multiband tight-binding theory and a quantum-mechanical approach to electronic transport. In this section we describe their model and main results which follow from this model.

The multiband description of the electronic structure within the tight-binding representation can be obtained by generalizing the single-band Hamiltonian (14.1) to include the valence $s, p$ and $d$ orbitals. We consider a perfect crystal which is divided into equivalent unit cells $m$ consisting of atomic sites $j$ with associated atomic wave functions $|m j \alpha\rangle$, where $\alpha$ denotes the orbital character Within the two-center, orthogonal tight-binding approximation the single particle Hamiltonian of the perfect system $H^{0}$ is determined by the on-site atomic energies $E_{j \alpha}$ and by the hopping integrals $\left\langle 0 j \alpha\left|H^{0}\right| m j^{\prime} \alpha^{\prime}\right\rangle$ (index 0 denotes the origin of the system). In order to solve the Schrödinger equation

$$
H^{0}|\eta \mathbf{k}\rangle=E_{\eta}(\mathbf{k})|\eta \mathbf{k}\rangle
$$

the eigenstate $|\eta \mathbf{k}\rangle$ with band index $\eta$ and wave vector $\mathbf{k}$ can be expanded in terms of the atomic functions:

$$
|\eta \mathbf{k}\rangle=\frac{1}{\sqrt{N}} \sum_{m j \alpha} e^{i \mathbf{k}\left(\mathbf{R}_{m}+\mathbf{r}_{j}\right)} c_{\eta, j \alpha}(\mathbf{k})|m j \alpha\rangle,
$$

where $N$ is the total number of cells in the crystal, $\mathbf{R}_{m}$ is the $m$-th lattice vector and $\mathbf{r}_{j}$ is the basis vector (a site position within the unit cell). The expansion coefficients $c_{\eta, j \alpha}(\mathbf{k})$ are obtained by solving the tight-binding secular equation

$$
\sum_{j^{\prime} \alpha^{\prime}} H_{j \alpha, j^{\prime} \alpha^{\prime}}^{0}(\mathbf{k}) c_{\eta, j^{\prime} \alpha^{\prime}}(\mathbf{k})=E_{\eta}(\mathbf{k}) c_{\eta, j \alpha}(\mathbf{k}),
$$

where the matrix elements of the Hamiltonian are defined by

$$
H_{j \alpha, j^{\prime} \alpha^{\prime}}^{0}(\mathbf{k})=\sum_{m} e^{i \mathbf{k}\left(\mathbf{R}_{m}+\mathbf{r}_{j^{\prime}} \mathbf{r}_{j}\right)}\left\langle 0 j \alpha\left|H^{0}\right| m j^{\prime} \alpha^{\prime}\right\rangle \text {. }
$$

The tight-binding parameters, i.e. hopping integrals and the on-site atomic energies, can be obtained by fitting the tight-binding bands of the elemental metals to the bands computed from firstprinciples. ${ }^{158}$ This makes it possible to describe realistically the band structure of magnetic multilayers taking into account $s, p$ and $d$ orbitals, their full hybridization and spin-polarization Matrix elements of the velocity operator, which are necessary for calculating the conductivity, can be found from the relation $\hbar \mathrm{v}^{\mu}(\mathbf{k})=\partial H^{0}(\mathbf{k}) / \partial \mathrm{k}^{\mu}$.

The next step is including defect scattering in the model. We assume that the total Hamiltonian of the disordered system can be represented as the sum of the perfect Hamiltonian $H^{0}$ and the scattering potential $V, H=H^{0}+V$. The scattering potential which reflects the presence of intrinsic defects within the multilayer is assumed to effect the on-site atomic energy levels randomly so that

$$
\left\langle m j \alpha|V| m^{\prime} j^{\prime} \alpha^{\prime}\right\rangle=V_{m j \alpha} \delta_{m m^{\prime}} \boldsymbol{\delta}_{i j} \boldsymbol{\delta}_{\alpha \alpha^{\prime}} .
$$

Here for simplicity the scattering potential is taken to be diagonal with respect to the cell, site and orbital indices. The diagonal elements $V_{m j \alpha}$ are taken to be randomly distributed such that

$$
\begin{aligned}
& \left\langle V_{m j \alpha}\right\rangle=0, \\
& \left\langle V_{m j \alpha} V_{m j^{\prime} \alpha^{\prime}}\right\rangle=\gamma^{2} \delta_{m m^{\prime}} \delta_{j j^{\prime}} \delta_{\alpha \alpha^{\prime}},
\end{aligned}
$$

where $\langle\ldots\rangle$ denotes the configuration average and $\gamma^{2}$ is the mean square displacement of the on-site atomic energies. The parameter $\gamma$ is a parameter in the theory. It characterizes the degree of disorder within the system reflecting the scattering by defects and can be chosen to provide a realistic saturation resistivity of the multilayer.

We stress that in general the scattering potential, which is produced by a particular type of defect or impurity in a magnetic system, is expected to be spin-dependent. However, GMR structures are characterized by various types of defects. Physically this means that the spin asymmetry and the strength of the scattering potential can vary from defect to defect. This fact is supported by firstprinciple calculations, ${ }^{180}$ which show a different spin dependence of scattering produced by stacking faults, twin boundaries and $\mathrm{Cu}$ impurities in bulk $\mathrm{Co}$. On average, it is reasonable to assume no spin dependence in the scattering potential, which in our model implies a spin-independent parameter $\gamma$ Note that as we will see below the scattering rate remains spin-dependent as the result of the spin asymmetry in the density of states at the Fermi level.

The zero-temperature conductivity per spin is expressed by the Kubo-Greenwood formula (13.10), which requires configurational averaging as was described in section 13. This averaging can be performed in the weak scattering limit, which is justified for GMR structures. Indeed, it is wellknown that within the single-band model the weak scattering limit is valid provided the random potential is such that $\gamma \ll \Delta$, where $\Delta$ is the bandwidth (e.g., ref.126). This expression may be generalized to the case of the multiband system as $m\left(E_{F}\right)<<1$, where $n\left(E_{F}\right)$ is the average density of states per orbital at the Fermi energy. We will find that in order to obtain typical experimental values of the resistivity of the $3 d$-metal multilayer structures, i.e. $1-100 \mu \Omega \mathrm{cm}$, one needs $\gamma$ to be in the range from 0.1 to $1 \mathrm{eV}$. Since $n\left(E_{F}\right)$ is of the order of $0.1 \mathrm{eV}^{-1}$, the weak scattering approximation is justified. In the weak scattering limit the self-energy, which enters equation (13.12) for the configuration-averaged Green's function $\langle G\rangle$, is determined by $\Sigma=\left\langle V G_{0} V\right\rangle .{ }^{126}$ Using the properties of the scattering potential defined by equations (17.5-17.7) and performing the averaging explicitly, we obtain

$$
\Sigma_{j \alpha, j^{\prime} \alpha^{\prime}}\left(E_{F}\right)=\gamma^{2} \sum_{\eta} \int \frac{\Omega d \mathbf{k}}{(2 \pi)^{3}} \frac{\left|c_{\eta, j \alpha}(\mathbf{k})\right|^{2}}{E_{F}-E_{\eta}(\mathbf{k})+i \varepsilon} \delta_{i j j^{\prime}} \delta_{\alpha \alpha^{\prime}},
$$


where $\Omega$ is the volume of the unit cell. It follows that the imaginary part of the self-energy will be proportional to the partial density of states at the Fermi energy $n_{j \alpha}\left(E_{F}\right)$ related to site $j$ and orbital $\alpha$ In particular, we can define the partial scattering rates by

$$
\tau_{j \alpha}^{-1}=\frac{2}{\hbar} \operatorname{Im} \Sigma_{j \alpha, j \alpha}\left(E_{F}\right)=\frac{2 \pi}{\hbar} \gamma^{2} n_{j \alpha}\left(E_{F}\right),
$$

which are spin-dependent due to the spin-dependence in the densities of states.

The final expression for the conductivity takes the form

$$
\sigma^{\mu v}=\sum_{j \alpha} \sigma_{j \alpha}^{\mu v}=\sum_{j \alpha j^{\prime} \alpha^{\prime}} \frac{e^{2}}{\pi \hbar} \int \frac{d \mathbf{k}}{(2 \pi)^{3}} \Lambda_{j \alpha, j^{\prime} \alpha^{\prime}}^{\mu}(\mathbf{k}) \Lambda_{j^{\prime} \alpha^{\prime}, j \alpha}^{{ }^{\prime}(\mathbf{k})},
$$

where the mean free path operator $\Lambda^{\mu}(\mathbf{k})$ is defined by

$$
\Lambda^{\mu}(\mathbf{k})=\hbar \mathrm{v}^{\mu}(\mathbf{k}) \operatorname{Im}\left[E_{F}-H^{0}(\mathbf{k})-\Sigma\left(E_{F}\right)\right]^{-1}
$$

and the self-energy is given by formula (17.8). The partial conductivities $\sigma_{j \alpha}^{\mu v}$ corresponding to site $j$ and orbital $\alpha$ can be used to determine the local (layer-dependent) contributions to the conductivity from non-equivalent atoms (layers) $j$

$$
\sigma_{j}^{\mu v}=\sum_{\alpha} \sigma_{j \alpha}^{\mu v}
$$

and the partial contributions from the $s, p$ and $d$ orbitals

$$
\sigma_{l}^{\mu v}=\sum_{\alpha \in l} \sum_{j} \sigma_{j \alpha}^{\mu v},
$$

where $l$ denotes $s, p$ or $d$ orbitals.

Fig.31a illustrates the model by applying it to calculating the conductivity of bulk $f c c \mathrm{Cu}$ as a function of energy, or equivalently as a function of the Fermi energy within the rigid band approximation. Comparing the solid line in the bottom panel of Fig.31a to the DOS in the top panel in Fig.31a, we see that the conductivity of $\mathrm{Cu}$ is strongly suppressed within the $d$ bands $(-5 \mathrm{eV}<E<$ $1.5 \mathrm{eV})$. The decomposition of the conductivity into its partial contributions shows that in this interval of energies the conductivity is mainly determined by the $d$ electrons, the weight of the $s p$ character varying from about $10 \%$ to $30 \%$. The very low contribution of the $s p$ electrons to the conductivity within the $d$ bands is connected with the strong hybridization between the $s p$ electrons and the $d$ electrons, which flatten the $s p$ bands reducing their velocity. Outside the $d$ band the conductivity rapidly increases and the contribution of the $s p$ electrons becomes dominant. The Fermi level in bulk copper lies high above the $d$ bands, so that most of the current is carried by the $s p$ electrons, the $d$ contribution being just $3 \%$

As is evident from Fig.31b, the $d$ bands of Co are exchange-split due to ferromagnetism, which makes the contributions of the two spins to the conductivity different. Similar to $\mathrm{Cu}$, for energie within the $d$ bands the conductivity is low and mainly determined by the $d$ electrons. Above and below the $d$ bands the conductivity rapidly increases with a crossover from mainly $d$ to mainly $s p$ contributions (not shown). The Fermi level in Co lies above the top of the $d$ band for the majorityspin electrons but falls inside the $d$ band for the minority-spin electrons. This results in the sizeable difference between the conductivities for the two spins at the Fermi energy. As can be seen from the bottom panel in Fig. 31b, $\sigma^{\uparrow}$ exceeds $\sigma^{\downarrow}$ by a factor of five. Note that the spin asymmetry in the conductivity $\alpha=\sigma / \sigma$ depends on the degree of disorder $\gamma$. We also note that the spin asymmetry is opposite to the prediction in the ballistic regime (see Fig.27b), according to which the minority-spin conductance in $\mathrm{Co}$ is higher than the majority-spin conductance.
The results of these model calculations for the bulk metals show that the band structure plays a very important role in the conductivity. In particular, (i) the contribution from the $d$ electrons is sizeable and in general can not be neglected; (ii) the $s p$ - $d$ hybridization is important due to reducing the conductivity of the $s p$ electrons within the $d$ bands; and (iii) the exchange splitting of the $d$ bands results in the strong spin asymmetry of the conductivity at the Fermi energy.
Energy $(\mathrm{eV})$

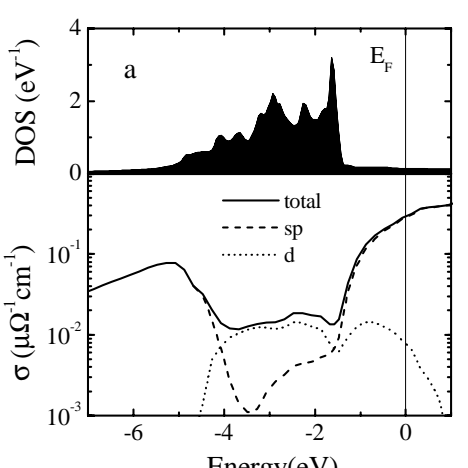

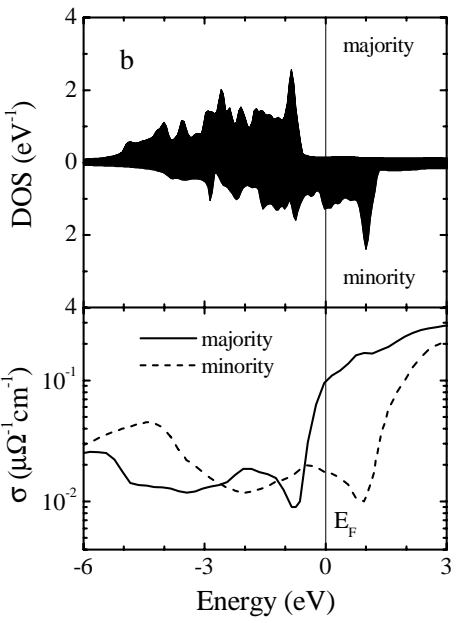

Energy $(\mathrm{eV})$
Fig.31 Density of states (top panels) and conductivity (bottom panels) as a function of electron energy for bulk $f c c \mathrm{Cu}$ (a) and for bulk $f c c \mathrm{Co}$ (b). Conductivity is calculated for $\gamma=0.5 \mathrm{eV}$ and decomposed into the contributions from the $s p$ and $d$ electrons for $\mathrm{Cu}$ and from the majority and minority spins for $\mathrm{Co}$. The vertical line shows the position of the Fermi level. After Tsymbal and Pettifor.

All these effects manifest themselves in the GMR multilayers. Fig.32a shows the density of state (the top panel), conductivity (the middle panel) and GMR (the bottom panel) of a $\mathrm{Co}_{4} / \mathrm{Cu}_{4}(001)$ multilayer calculated as a function of energy. Similar to bulk Co, the DOS of the parallel-aligned $\mathrm{Co} / \mathrm{Cu}$ multilayer is asymmetric between the majority and minority spins. This is reflected in the conductivity $\sigma$. For the energies that lie within the $d$ band the $s p$ electrons are slowed down by the hybridization with the $d$ electrons and the conductivity is low. Above the top of the $d$ bands $\sigma$ increases rapidly. This increase is due to the "acceleration" of the $s p$ electrons which are less affected by the $s p-d$ hybridization above the $d$ bands. The top of the $d$ bands for the majority spins lies at approximately $0.5 \mathrm{eV}$ below the Fermi energy and for the minority spins at about $1 \mathrm{eV}$ above the Fermi energy. Therefore, in the interval of the energies from $-0.5 \mathrm{eV}$ to $1 \mathrm{eV}$ the difference between $\sigma^{\uparrow}$ and $\sigma$ is most pronounced. The presence of the $d$ levels up to $1 \mathrm{eV}$ above $E_{F}$ for the AP configuration makes the conductivity per spin in this case similar to the conductivity of the minority spin electrons for the $\mathrm{P}$ configuration. This results in the crucial difference between the $\sigma_{P}$ and $\sigma_{A P}$ and, consequently, in GMR. As seen from the bottom panel in Fig.32a the large values of GMR are predicted only in the interval of energies between the top of the majority-spin $d$ band and the top of the minority-spin band. As the Fermi level in $\mathrm{Co} / \mathrm{Cu}$ multilayers lies within this interval a sizeable value of GMR can be observed in this system. 
An interesting effect, which is evident from the bottom panel in Fig.32a, is that GMR in the $\mathrm{Co} / \mathrm{Cu}$ system increases with increasing energy above the Fermi level. A pronounced peak in $\Delta R / R$ appears for hot electrons at about $1 \mathrm{eV}$ above the Fermi level, taking the large value of nearly $400 \%$ for the CPP geometry. The enhancement of GMR in $\mathrm{Co} / \mathrm{Cu}$ multilayers has been observed by Monsma et al 181 who have measured more than $390 \%$ CPP magnetocurrent change in a "spin-valve transisto". The energy of the hot electrons in this device was defined by the Schottky barrier height of about $0.7 \mathrm{eV}$ for the collector and about $0.6 \mathrm{eV}$ for the emitter. The results of those calculations give a clear interpretation of this enhanced GMR effect, which originates from the increasing spin asymmetry in the electron velocities for hot electrons up to $1 \mathrm{eV}$ above the Fermi energy. We note, however, that an accurate quantitative description of the spin-vale transistor requires including nonmagnetocurrent of $560 \%$ at $100 \mathrm{~K}$ has been measured by Jansen et al ${ }^{182}$ in a spin valve transistor with a $\mathrm{Ni}_{80} \mathrm{Fe}_{20} / \mathrm{Au} / \mathrm{Co}$ base.

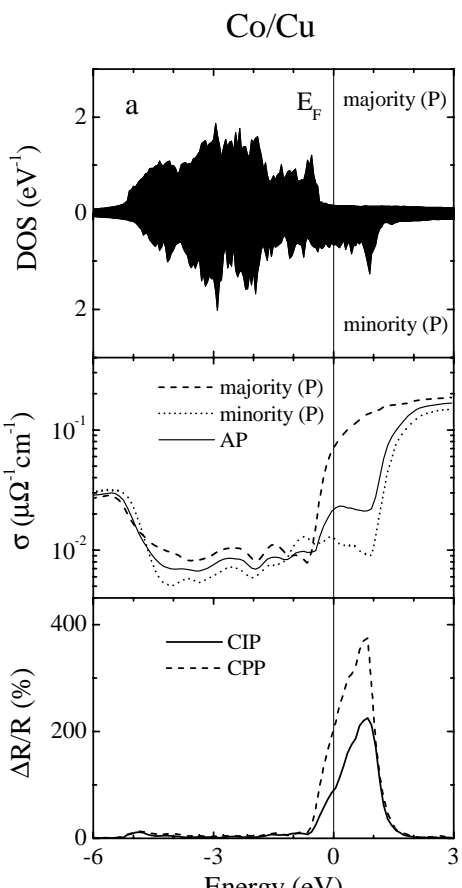

Energy (eV)
$\mathrm{Fe} / \mathrm{Cr}$

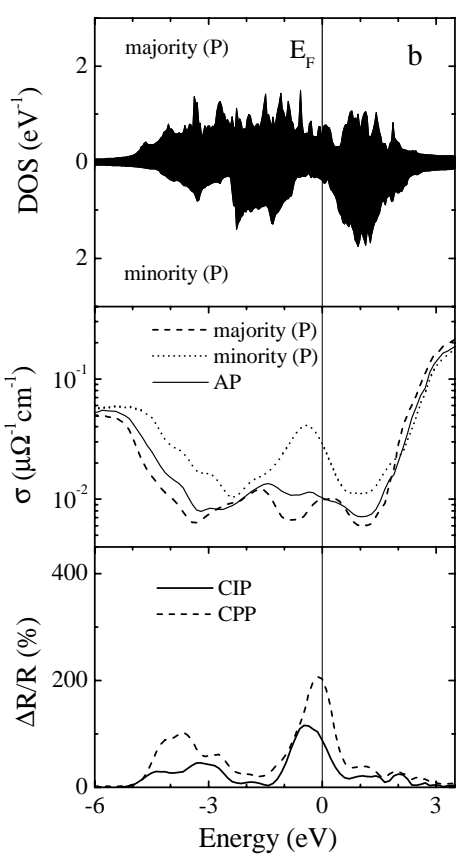

Fig.32 Density of states (top panels), conductivity (middle panels) and GMR (bottom panels) of the $\mathrm{Co}_{4} / \mathrm{Cu}_{4}$ (a) and $\mathrm{Fe}_{4} / \mathrm{Cr}_{4}$ (b) multilayers as a function of electron energy calculated within the tight-binding approximation. The vertical line shows the position of the Fermi level. After Tsymbal and Pettifor. ${ }^{17}$

The $\mathrm{Fe} / \mathrm{Cr}$ system behaves differently from the $\mathrm{Co} / \mathrm{Cu}$ system. As is evident from Fig.32b the Fermi level in the $\mathrm{Fe}_{4} / \mathrm{Cr}_{4}$ multilayer lies within the $d$ bands for both spin orientations. However, the
DOS exhibits a pronounced valley for the minority spins, with the Fermi level lying almost at the bottom of this valley, which follows from the similarity of the minority DOS of Fe and the DOS of $\mathrm{Cr}$. We note that the presence of this valley in the minority DOS of bulk Fe makes the minority-spin conductivity higher than the majority-spin conductivity, i.e. $\alpha=\sigma^{\uparrow} / \sigma^{\downarrow}<1$, which is opposite to bulk Co. ${ }^{179}$ For the parallel-aligned multilayer the $\sigma$ displays an enhancement for electron energies lying in the region of this valley (see the dotted line in the middle panel of Fig.32b). On the contrary, the $\sigma$ for the P orientation and the conductivity for the AP orientation do not change essentially in this interval of energies (the dashed and the solid lines in the middle panel of Fig.32b). This results in the difference between $\sigma_{P}$ and $\sigma_{A P}$ and, consequently, in GMR for the energies close to the Fermi leve (the bottom panel of Fig.32b). The variation of GMR as a function of energy shows that contrary to the $\mathrm{Co} / \mathrm{Cu}$ multilayers GMR decreases above the Fermi level.

The magnitude of GMR decreases with increasing disorder. As is seen from Fig. 33 the value of GMR in a $\mathrm{Co}_{4} / \mathrm{Cu}_{4}$ multilayer drops by an order of magnitude, i.e. from about $105 \%$ to $15 \%$ as the disorder parameter $\gamma$ changes from $0.4 \mathrm{eV}$ to $1.2 \mathrm{eV}$. The corresponding saturation resistivity varie from $8 \mu \Omega \mathrm{cm}$ to $55 \mu \Omega \mathrm{cm}$ (Fig.33a), spanning the range of experimental values. This drop in GMR with increasing disorder has an important underlying physics. ${ }^{179}$ It is related to the interban transitions driven by the applied electric field, which makes the quantum mechanical description different from the semiclassical approach. In metals, in the absence of disorder these transitions may be important only in an exceedingly small region of $\mathbf{k}$-space near points where two bands become degenerate.$^{30}$ In the presence of disorder, however, the electronic levels are broadened by the value of $\operatorname{Im} \Sigma$. If this broadening becomes comparable with the distance between bands even an infinitesima small electric field can lead to the interband transitions. In this case, the semiclassical approximation breaks down.

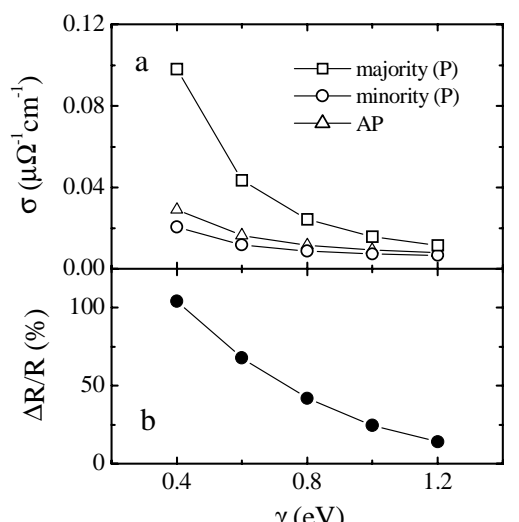

Fig.33 Conductivity of the $\mathrm{Co} / \mathrm{Cu}$ multilayer as a function of the root-mean-square variation of the on-site atomic energies $\gamma$, which characterizes the degree of disorder within the multilayer. After Tsymbal and Pettifor.

The drop of GMR in the Co/Cu multilayer within increasing disorder, which is shown in Fig.33b, can, therefore, be explained as follows. The conductivity of the parallel-aligned multilayer is mainly determined by the majority-spin electrons, which have predominantly free-electron-like $s p$ character 
due to the position of $E_{F}$ above the $d$ bands. Therefore, with increasing $\gamma \sigma_{P}$ decreases approximately as $1 / \gamma^{2}$. On the other hand, the conductivity of the antiparallel-aligned multilayer is primarily due to the $d$ electrons because $E_{F}$ falls inside the $d$ band for this alignment. In this case, due to the interban transitions between the $s, p$ and $d$ levels, the $\sigma_{A P}$ decreases more slowly than $1 / \gamma^{2}$ so that GMR drops with increasing $\gamma$. This dependence is not predicted by the semiclassical treatment of conductivity, according to which the conductivity for both the $\mathrm{P}$ and AP alignments is inversely proportional to $\gamma^{2}$ so that GMR is independent of $\gamma$. Within the semiclassical approach the GMR values found for a $\mathrm{Co}_{4} / \mathrm{Cu}_{4}$ multilayer are about $400 \%$ for the CIP geometry and about $1000 \%$ for the CPP geometry. These values are much higher than those predicted within the quantum description (Fig.33b) and those observed experimentally (section III). We note that the interband transitions can also contribute to the reduction of GMR with temperature (section 10).

The predicted values of GMR for the $\mathrm{Co} / \mathrm{Cu}$ and $\mathrm{Fe} / \mathrm{Cr}$ multilayers are in good agreement with experiment. For example, GMR measured in $\mathrm{Fe} / \mathrm{Cr}$ multilayers at $\mathrm{T}=4.2 \mathrm{~K}$ was found to be $79 \%$ for relatively thin $\mathrm{Cr}$ layers of $0.9 \mathrm{~nm}$. ${ }^{1}$ For the $\mathrm{Fe}_{4} / \mathrm{Cr}_{4}$ multilayer which has a similar $\mathrm{Cr}$ thickness and for the disorder parameter $\gamma=0.6 \mathrm{eV}$ which provides a similar saturation resistivity of $32 \mu \Omega \mathrm{cm}$ (compared to the experimental resistivity of $31 \mu \Omega \mathrm{cm}$ ), the calculated result of $67 \%$ is consistent with that measured experimentally. Another example is GMR in $\mathrm{Co} / \mathrm{Cu}$ multilayers which was found to be $120 \%$ at $\mathrm{T}=4.2 \mathrm{~K} .^{38}$ The thicknesses of the $\mathrm{Co}$ and $\mathrm{Cr}$ layers in these experiments were $1 \mathrm{~nm}$ and $0.9 \mathrm{~nm}$ respectively and the saturation resistivity was about $10 \mu \Omega \mathrm{cm}$. The model predicts that the $\mathrm{Co}_{4} / \mathrm{Cr}_{4}$ multilayer with $\gamma=0.4 \mathrm{eV}$ (the saturation resistivity of $8 \mu \Omega \mathrm{cm}$ ) has GMR of $105 \%$ which is also very close to the experimental value. There is, however, an exception. The model is not able to explain the high GMR value of $220 \%$ measured Schad et al..$^{39,70}$ on high-quality epitaxial $\mathrm{Fe} / \mathrm{Cr}$ multilayers (see also section 7). As was emphasized by Schad et al., the high magnitudes of GMR were only observed for ultrathin magnetic layers, which display an enhanced density of steps at the interfaces and at very low temperatures. The spin-dependent scattering potentials produced by the interfacial roughness are obviously important in this case, but have not been included in the above theoretical model.

Layer-dependent conductivity $\sigma_{j}$ defined by equation (17.12) provides important information about contributions to the spin current and GMR from different layers. ${ }^{183}$ Fig. 34 shows results for $\mathrm{Co}_{16} / \mathrm{Cu}_{10} / \mathrm{Co}_{16}$ trilayer in which the thicknesses of the $\mathrm{Co}$ and $\mathrm{Cu}$ layers, namely $3 \mathrm{~nm}$ and $2 \mathrm{~nm}$ respectively, are representative of the experiments on spin valves. The calculations were performed assuming a bulk scattering with $\gamma=0.7 \mathrm{eV}$ that gives a saturation resistivity of $23 \mu \Omega \mathrm{cm}$ and a GMR of experimental data by Egelhoff $e t c$ parallel $(\mathrm{P})$ configuration the local conductivity of the Co layers is much higher for the majority spins than for the minority spins, which reflects the spin-dependent conductivity in the bulk Co (see Fig.31b at $E=E_{F}$ ). The local conductivities $\sigma_{C u}$ in the $\mathrm{Cu}$ layers are higher than those in the Co layers and are different for different spins. This is because the transport properties of electrons propagating in the $\mathrm{Cu}$ layer are affected strongly by the spin-dependent scattering in the adjacent Co layers. Due to the much higher minority-spin DOS at the Co sites, electrons contributing to the conductivity in the $\mathrm{Cu}$ laye scatter more strongly in the minority channel and, therefore, $\sigma_{C u}^{\uparrow}>\sigma_{C u}^{\downarrow}$. As is seen from the open symbols in Fig.34a, for the antiparallel (AP) magnetizations there is an asymmetry in the local conductivities of $\mathrm{Cu}$ and $\mathrm{Co}$. The $\sigma_{C o}$ in the local minority-spin channel is almost the same for the AP configuration as for the $\mathrm{P}$ configuration. On the other hand, the $\sigma_{C o}$ in the local majority-spin channel are reduced because the majority carriers can propagate through the $\mathrm{Cu}$ layer and scatter in the opposite Co layer which has a high local minority-spin DOS. As can be seen from Fig.34b, thi mechanism leads to GMR, which comes from electrons contributing to the conductivity in the Co layers. Surprisingly, a significant contribution to GMR originates from the $\mathrm{Cu}$ spacer layer, the local magnetoconductance being highest near the interfaces. As we will see in the next section, these enhanced values of GMR can partly be explained by electron channeling within the $\mathrm{Cu}$ spacer layer
The enhancement of magnetoconductance in the spacer layer and at the interfaces was also found for $\mathrm{Fe} / \mathrm{Cr} / \mathrm{Fe}$ spin valves. ${ }^{183}$

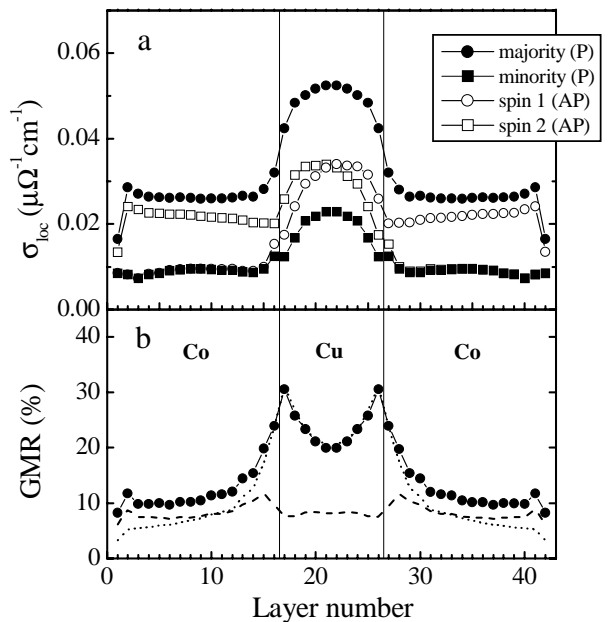

Fig.34 Layer-dependent conductivity (a) and magnetoconductance (b) in the $\mathrm{Co}_{16} / \mathrm{Cu}_{10} / \mathrm{Co}_{16}(001)$ spin valve in the presence of bulk scattering with $y=0.7 \mathrm{eV}$ (symbols). The magnetoconductance is defined as $\left(\sigma_{j}^{P}-\sigma_{j}^{A P}\right) / \sigma^{A P}$, where $\sigma_{j}^{P}$ and $\sigma_{j}^{A P}$ are the local conductivities for the $\mathrm{P}$ and AP configurations at the layer $j$. The dotted and dashed lines in figure $b$ show respectively the magnetoconductance for enhanced outer-boundary scattering with $\gamma_{\text {boundary }}=2.8 \mathrm{eV}$ and enhanced interface scattering with $\gamma_{\text {interface }}=2.8 \mathrm{eV}$. After Tsymba and Pettifor. ${ }^{183}$

The local contributions to GMR are very sensitive to the properties of the interfaces between the ferromagnetic and the spacer layers. As can be seen from the dashed line in Fig.34b, increasing disorder at the interfaces results in the strong reduction of the contribution to GMR from the space layer and at the interfaces. A strong reduction of GMR was also found if a magnetically-dead (paramagnetic) Co layer is put at the interface. ${ }^{183}$ Contrary to interface disorder, disorder at the outerboundaries of the spin valve reduces the contribution to GMR only from the Co layers, especially from those layers which are close to the outer boundaries (the dotted line in Fig.34b). This is also the case for paramagnetic Co layers at the outer-boundaries (not shown). The latter fact suggests that using Ta as a buffer layer or FeMn as a pinning layer is not favorable for obtaining large values of GMR in spin valves, because these metals have a high density of states at the Fermi level due to the $d$ band contribution.

An accurate description of the band structure is crucial to elucidate the striking features in the experimental thickness-dependent conductivity measured in-situ in $\mathrm{NiO} / \mathrm{Co} / \mathrm{Cu} / \mathrm{Co}$ spin valves by Bailey et al., ${ }^{112}$ which have been described in section 12 and shown in Fig.22a. The experimental data are the consistent with strong scattering of the conduction electrons in $\mathrm{Cu}$ at the interfaces with $\mathrm{Co}$ due to a high density of empty Co $d$ states at the Cu boundaries. This scattering explains both the positive curvature of the conductance as a function of the $\mathrm{Cu}$ layer thickness when $\mathrm{Cu}$ is placed on the $\mathrm{NiO} / \mathrm{Co}$ bilayer and the conductance drop when the Co layer is placed on top of the $\mathrm{NiO} / \mathrm{Co} / \mathrm{Cu}$ trilayer. The results of the comparison of the experimental data with the calculations are shown in 
Figs.35a,b. The positive curvature in the conductance versus $\mathrm{Cu}$ thickness $d_{C u}$ displayed in Fig. 35a is the result of the decreasing scattering of electrons in the $\mathrm{Cu}$ layers located far from the interface with the bottom Co layer. The conductance drop shown in Fig. $35 \mathrm{~b}$ is a consequence of the reduction of the local conductance in the $\mathrm{Cu}$ layer when the Co layer is placed on top. The conductance drop increase within the $\mathrm{Cu}$ layer thickness because for the larger $d_{C u}$ the local conductance in the $\mathrm{Cu}$ layer becomes less effected by the scattering at the bottom Co layer. As is seen from Fig.35b, the agreement between the experimental data and the calculation is improved if additional disorder is introduced to the top $\mathrm{Cu} / \mathrm{Co}$ interface, which is attributed to the segregation of low surface energy $\mathrm{Cu}$ through $\mathrm{Co}$ in the experiment. $^{112}$
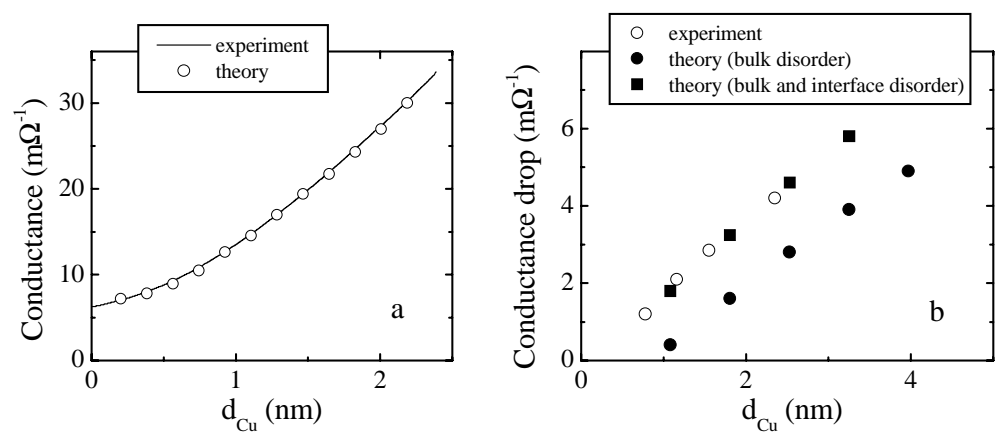

Fig.35 Comparison of experimental and theoretical results for the conductance in $\mathrm{NiO} / \mathrm{Co} / \mathrm{Cu}\left(d_{\mathrm{Cu}}\right)$ films (a) and for the conductance drop in $\mathrm{NiO} / \mathrm{Co} / \mathrm{Cu}\left(d_{\mathrm{Cu}}\right) / \mathrm{Co}$ spin valves (b) as a function of the Cu layer thickness $d_{0}$. The experimental curve in figure $a$ has been offset by $23 \mathrm{~m} \Omega^{-1}$. In figure $b$ the a reement is improved if additionl disorder is introduced to the top $\mathrm{Cu} / \mathrm{Co}$ interface that is attributed to segegtion of low suface energy $\mathrm{Cu}$ through $\mathrm{Co}$ in the interface that is attributed to segregation of low surface energy Cu through Co in the
experiment. After Bailey et al. ${ }^{112}$

The decisive role of the spin-polarized band structure for spin-dependent transport in magnetic layered systems is supported by studying the thermoelectric power (TEP) in $\mathrm{Co} / \mathrm{Cu}$ and $\mathrm{Fe} / \mathrm{Cr}$ multilayers Tsymbal et al. ${ }^{184}$ Experimental studies show that TEP is positive in $\mathrm{Fe} / \mathrm{Cr}$ magnetic multilayers. ${ }^{185}$ The magneto-thermoelectric power (MTEP), i.e. the change in TEP associated with an applied magnetic field, is also positive at room temperature. On the contrary, as was confirmed by numerous experimental studies, ${ }^{186}$ both TEP and MTEP are negative in $\mathrm{Co} / \mathrm{Cu}$ multilayers. According to the Mott formula the TEP is related to the change in the conductivity at the Fermi energy ${ }^{187}$

$$
S=-\left.\frac{\pi^{2} k^{2} T}{3 e} \frac{\partial \ln \sigma}{\partial E}\right|_{E_{F}}
$$

As is seen from the middle panels in Figs.32a,b, for both parallel and antiparallel magnetizations a the Fermi energy the conductivity of the $\mathrm{Co} / \mathrm{Cu}$ multilayer increases with increasing energy whereas the conductivity of the $\mathrm{Fe} / \mathrm{Cr}$ multilayer decreases. According to the Mott formula (17.14) this implies that the thermoelectric power is negative in the $\mathrm{Cu} / \mathrm{Cu}$ multilayers whereas it is positive in the $\mathrm{Fe} / \mathrm{Cr}$ multilayers. This theoretical prediction is in agreement with experimental observations. ${ }^{185,186}$ Tsymbal et al. ${ }^{184}$ have also shown that their model accounts for the experimentally observed sign of the magneto-thermoelectric power at room temperature, when the magnetizations of the consecutive ferromagnetic layers change their alignment.

We see, therefore, that the model for GMR, which incorporates spin-independent disorder in the on-site atomic energy levels and a realistic spin-dependent electronic structure within a tight-binding representation of electronic transport, provides a reliable approach for studying GMR in magnetic multilayers and spin valves. It accounts for the experimentally observed values of GMR in $\mathrm{Co} / \mathrm{Cu}$ and $\mathrm{Fe} / \mathrm{Cr}$ magnetic multilayers and shows that the effects of the electronic band structure, such as it spin-polarization, $s p-d$ hybridization and the contribution of the $d$ electrons to the conductivity, are crucial for the realistic description of conductivity and GMR in magnetic layered systems.

\section{First-principle models}

First-principle models for the electronic structure in conjunction with the quantum-mechanical formulation of the electronic transport become a powerful tool to study the conductivity and GMR in magnetic layered systems. On the one hand, the major advantage of the first-principle approach is the possibility to treat the electronic structure in a self-consistent way taking into account the charge and spin transfer at the interfaces. On the other hand, as we saw in the previous section, the quantummechanical formulation of the transport theory is necessary for the realistic modeling of GMR.

The principal challenge to the ab-initio simulations lies in a realistic modeling of the scattering As was discussed in section 16, assuming that interdiffusion at the interfaces is the only mechanism of scattering in magnetic multilayers results in highly overestimated values of GMR. A reliable approach must include other contributions to the resistivity, such as from intrinsic structural defects which were considered in the previous section.

Unfortunately, proper first-principle treatment of all the possible mechanisms of scattering in layered structures is very complicated. Realizing this, Butler et al ${ }^{188}$ use a simplified approach. Arguing that in realistic GMR systems scattering comes from several sources such as impurities, grain boundaries, vacancies, voids, and phonons, they approximate these scattering processes by a phenomenological local scattering rate, which could be in general spin-dependent. They use the Kubo-Greenwood formula for the conductivity in which they average the two Green's function independently, thereby neglecting the vertex corrections. They assume that the effect of this averaging is that each atomic potential acquires an imaginary term that describes the scattering rate in its vicinity.

Butler et al. introduce a non-local site-dependent conductivity $\sigma_{i j}^{\mu v}$ which determines the electrical current at site (layer) $i, \mathbf{j}_{i}$, related to the local electric field at site (layer) $j, \mathcal{E}_{j}$,

$$
j_{i}^{\mu}=\sum_{j v} \sigma_{i j}^{\mu v} \mathcal{E}_{j}^{v},
$$

where it is assumed that $\mathbf{j}_{i}$ is the average of the current density over the atomic cell at that site and the local field $\mathcal{E}_{j}$ is a constant over each atomic cell. If the electric field is applied parallel to the layers (CIP geometry) the local fields are uniform by symmetry and equal to the average applied field. This allows obtaining the overall conductivity by summating over all layers according to

$$
\sigma^{\mu v}=\frac{1}{d} \sum_{i j} d_{i} \sigma_{i j}^{\mu v}
$$

where $d_{i}$ is the thickness of layer $i$ and $d$ is the total film thickness.

The non-local conductivity can be found according to the Kubo-Greenwood formula

$$
\sigma_{i j}^{\mu v}=\frac{\hbar e^{2}}{\pi \Omega_{i}} \int_{\Omega_{i}} d \mathbf{r} \int_{\Omega_{j}} d \mathbf{r}^{\prime} \mathbf{v}^{\mu} \operatorname{Im}\left\langle G\left(\mathbf{r}, \mathbf{r}^{\prime}\right)\right\rangle v^{v} \operatorname{Im}\left\langle G\left(\mathbf{r}^{\prime}, \mathbf{r}\right)\right\rangle
$$


where the integration is performed over the atomic cells at sites $i$ and $j, \Omega_{i}$ and $\Omega_{j}$ being the volumes of the corresponding cells. The angular brackets denote the configurational average, which i introduced as a phenomenological imaginary part of the self-energy in the Green's function $G\left(\mathbf{r}, \mathbf{r}^{\prime}\right)$. In order to calculate $G\left(\mathbf{r}, \mathbf{r}^{\prime}\right)$ Butler et al. use the KKR formalism, which explicitly constructs the Green's function in terms of the local solutions of the Schrödinger equation and the scattering path operator of multiple scattering theory (see ref.188 and references therein). These are determined by the atomic potentials which are obtained self-consistently by using the local spin density approximation to density functional theory.

The non-local layer-dependent-conductivity is useful for a better understanding of the mechanism of magnetoresistance. An example of the calculation of the non-local conductivity for a $\mathrm{Co}_{7} / \mathrm{Cu}_{10} / \mathrm{Co}_{7}$ trilayer $^{188}$ is shown in Fig.36. In this calculation the phenomenological life-times are constrained to provide typical experimental resistivities for sputtered $\mathrm{Cu}$ and $\mathrm{Co}$ films. It is assumed that the scattering rate for the minority carriers in the bulk Co layers is 7 times that for the majority carries, reflecting the difference in the density of states in bulk Co. Due to intermixing at the interfaces the scattering rate of the minority spins at the interfacial layer is set 12 times that for the minority spins, the factor of 12 being based on coherent potential approximation calculations of the resistivity due to $\mathrm{Cu}$ impurities in $\mathrm{Co}$ and $\mathrm{Co}$ impurities in $\mathrm{Cu}$. In addition, strong spin-independent scattering at the outer boundaries of the trilayer is included.
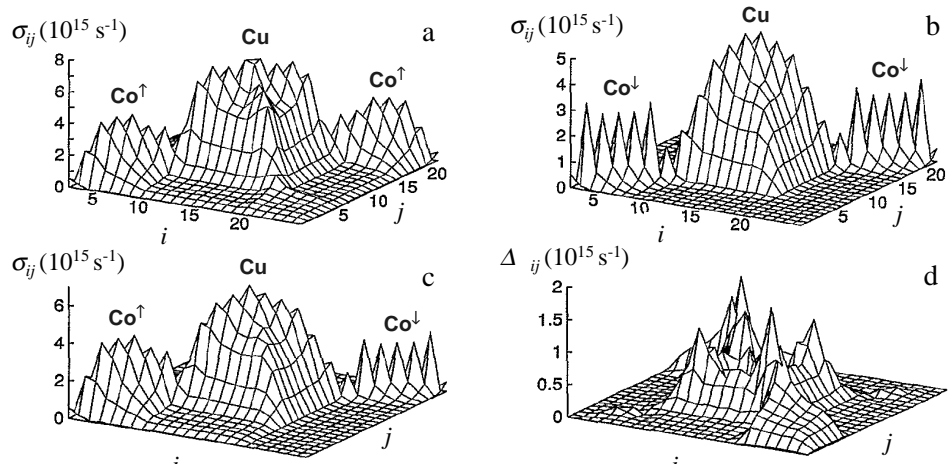

Fig.36 Non-local layer-dependent conductivities (in units $10^{15} \mathrm{~s}^{-1}=1113 \Omega^{-1} \mathrm{~cm}^{-1}$ ) for the Con-local layer-dependent conductivities (in units
$\mathrm{Co}_{7} / \mathrm{Cu}_{10} / \mathrm{Co}_{7}$ (111) trilayer: (a) majority channel for the parallel (P) alignment of
magnetizations, (b) minority channel for the $\mathrm{P}$ alignment, (c) spin channel, which is locally majority in the left side Co layer, for the antiparallel (AP) alignment, and (d) giant magnetoconductance, which is defined as the difference between the non-local conductivities for the $\mathrm{P}$ and $\mathrm{AP}$ alignments. The atomic layers are labeled by indices $i$ and j. After Butler et al. ${ }^{18}$

As is obvious from Fig.36a the conductivity is strongly non-local, which is reflected in large values of $\sigma_{i j}$ for $i \neq j$ especially within the $\mathrm{Cu}$ layers due to a long mean free path in $\mathrm{Cu} . \mathrm{A}$ comparison of Fig.36a with Fig.36b shows that the conductivities are "more local" for the minority carriers than for the majority carriers in Co because of a higher DOS and consequently a shorter mean free path for the former. As is seen from Figs.36a, b, and c, for both parallel and antiparalle alignments the conductivities have a tendency to be confined within the $\mathrm{Cu}$ or Co layers displaying a decrease at the interfaces. This "difficulty" in passing between layers results from the significant probability for reflection at the interfaces as a consequence of the potential steps created by the potential mismatch between $\mathrm{Co}$ and $\mathrm{Cu}$. As is evident from Fig.36d, a large contribution to GMR comes from the $\mathrm{Cu}$ spacer layer, which is a consequence of electron channeling and will be discussed below. Another contribution to GMR comes from the Co layers. This contribution arises from electrons that are accelerated in one Co layer, travel through the $\mathrm{Cu}$ layer to the other $\mathrm{Co}$ layer, and contribute to the current there. These show up in Fig.36d as the contributions in the near and far corners, which correspond to $i$ and $j$ being in the Co layers on opposite sides of the $\mathrm{Cu}$ spacer layer The layer-dependent conductivities (not shown), which are calculated from the non-local conductivities by $\sigma_{i}=\sum_{j} \sigma_{i j}$, are qualitatively similar to those calculated within the tight-binding model $^{183}$ (see Fig.34)

The presence of potential steps at the interfaces results in a contribution to GMR, which is distinct from spin-dependent scattering. This contribution originates from electron channeling as has been demonstrated within free-electron models ${ }^{144,135,136}$ Channeling can occur for the current parallel to the planes if a large portion of the electrons in a layer is specularly reflected from both it interfaces. If the scattering rate in that layer is lower than it is in neighboring layers, the reflected electrons in that layer see a lower effective scattering rate than they would in the absence of reflection. In magnetic multilayers, channeling contributes to GMR due to the electrons with parallel momenta which are strongly reflected for one spin, but not the other. In this case, channeling does not occur for electrons of either spin in the antiferromagnetic alignment because both transmit through one or the other interface. On the other hand, for the ferromagnetic alignment, electrons of one spin are confined to the layer, and if that layer has a lower scattering rate, these electrons cause a short circuit effect, thereby giving a contribution to GMR. ${ }^{18}$

Using first-principle calculations Butler et al. ${ }^{190}$ have shown that channeling can contribute to $\mathrm{GMR}$ in $\mathrm{Co} / \mathrm{Cu} / \mathrm{Co}$ spin valves. The majority-spin electrons in Co have a lower Fermi momentum than electrons in $\mathrm{Cu}$. This leads to the total internal reflection of those electrons whose momentum parallel to the interface exceeds the Fermi momentum of Co. These reflected electrons are, therefore, confined to the $\mathrm{Cu}$ layers, and, if the scattering rates are significantly lower in the $\mathrm{Cu}$ that in the $\mathrm{Co}$ they give a large contribution to the majority current and a large contribution to GMR. This contribution to GMR shows up in Fig. $36 \mathrm{~d}$ as a contribution to $\Delta \sigma_{i j}$ that is confined to the region near the center of the $i j$-plane where both $i$ and $j$ label $\mathrm{Cu}$ atomic layers.

The importance of channelling is nicely illustrated by Stiles ${ }^{189}$ who calculated the transmission probabilities across various interfaces. In this calculation the time-independent scattering states are determined by breaking space up into layers. ${ }^{191}$ The potential is computed for each layer from a bulk electronic structure calculation using the linearized-augmented-plane-wave method. Generalized Bloch states for a layer are computed from the potential in the layer. Generalized Bloch states are related to Bloch states by allowing the component of the wave vector normal to the interface to be complex. They form a complete set of states, which includes the usual Bloch states and all evanescent states, and consequently describe any time-independent solution of the Schrödinger equation for arbitrary boundary conditions. The generalized Bloch states for the two materials are matcher together across the interface to construct the electron scattering states, giving the reflection and transmission amplitudes directly.

Fig.37 shows the results of the calculations for transmission across $\mathrm{Cu} / \mathrm{Co}(001)$ interface. ${ }^{189} \mathrm{The}$ majority Fermi surface in $\mathrm{Co}$ is similar to the Fermi surface in $\mathrm{Cu}$, but is smaller. This similarity leads to the almost complete transmission of the majority-spin electrons from $\mathrm{Co}$ into $\mathrm{Cu}$ (the left bottom panel in Fig.37). The smaller size of the majority Co Fermi surface leads to the complete reflection for the states in $\mathrm{Cu}$ with the largest momenta parallel to the interface (the right top panel in Fig.37) These electrons contribute to channeling within the $\mathrm{Cu}$ layer in the $\mathrm{Co} / \mathrm{Cu} / \mathrm{Co}$ trilayer when magnetizations of the Co layers are parallel. ${ }^{190}$ Although the transmission of the minority-spin electrons is not simply characterized (due to the complicated nature of the minority Fermi surfaces in 
Co), it is seen from the right top panel in Fig. 37 that the same $\mathrm{Cu}$ states transmit much better into the minority Co states. Channeling by these electrons can give, therefore, a contribution to GMR if the scattering rate in $\mathrm{Cu}$ is much smaller than it is in $\mathrm{Co}$.

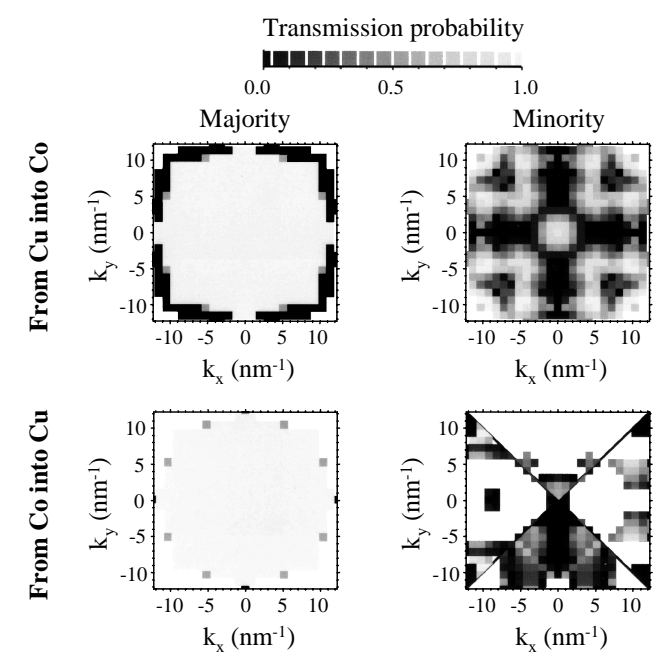

Fig.37 Spin-dependent transmission probabilities for the $\mathrm{Co} / \mathrm{Cu}(001)$ interface. The transmission probabilities are shown for various points on the Fermi surface projected onto the interface Brillouin zone. For minority Co, each sheet of the Fermi surface is shown in a fraction of the Brillouin zone. After Stiles. ${ }^{18}$

The degree of channeling which is observed in real experiments depends strongly on the detaile nature of the interfaces between the $\mathrm{Co}$ and $\mathrm{Cu}$ layers. Interfacial roughness can reduce or eliminate the contribution of channeling to GMR. ${ }^{190}$ As we have seen in the previous section, strong disorder the contribution of channeling to GMR. As we have seen in the previous section, strong disorder
(or a magnetically-dead Co layer) at the interfaces also results in a sizable reduction of the contribution to GMR from the spacer layer (the dashed line in Fig.34b). Evidence of channeling in a $\mathrm{Fe} / \mathrm{Au}(001)$ multilayer is found in experiments by Dekadjevi et al. ${ }^{192}$ which is consistent with the theoretical prediction of strong reflection of electrons propagating in $\mathrm{Au}$ from $\mathrm{Fe} / \mathrm{Au}$ interfaces. ${ }^{189}$

Using the model developed in ref.188, Butler et al. ${ }^{193}$ have performed calculations of the conductance and magnetoconductance in $\mathrm{Ta} / \mathrm{Co} / \mathrm{Cu} / \mathrm{Co} / \mathrm{FeMn} / \mathrm{Ta}$ spins valves and compared results with experimentally measured values as a function of the Co layer thickness. In the calculations, the conductance of the Ta and FeMn layers is neglected because their resistivities are very high, greate that $100 \mu \Omega \mathrm{cm}$. The interface between the Co and the Ta and between the Co and the FeMn is modeled by an atomic layer of Co with a very high scattering rate. The scattering rates within the $\mathrm{Co}$ and $\mathrm{Cu}$ layers are adjusted phenomenologically to be consistent with experimentally-measured resistivities of thick films. Various models for scattering are considered, such as spin-independent scattering, bulk scattering with different spin asymmetry in the scattering rates, interfacial spindependent scattering and both bulk and interface scattering. Butler et al. find that the magnetoconductance and its dependence on the Co layer thickness are consistent with calculation that include bulk spin-dependent scattering within the Co layers and some channeling of electrons in the $\mathrm{Cu}$ layer. They conclude that it is not necessary to invoke additional diffuse spin-dependent scattering at the interfaces to explain the experimental data. This is due to the strong spin-dependence of the transmission and reflection coefficients at the interfaces, which follow from the electronic band structures of $\mathrm{Co}$ and $\mathrm{Cu}$. This conclusion is consistent with the prediction of the tight-binding mode by Tsymbal and Pettifor. ${ }^{179,183}$

All the models considered above assume electrical transport in parallel within the two spinconduction channels, the total conductivity being the sum of the spin conductivities. Strictly speaking this is not the case due to the spin-orbit interaction. The spin-orbit interaction is a relativistic effect, which couples the electronic and the spin degrees of freedom and therefore makes the two-curren model inapplicable. Although the spin-orbit interaction is relatively small in the transition $3 d$ metals and does not effect strongly the electronic structure of these metals, it can affect transport properties of bulk alloys, for example, avoiding the short circuits that can arise in either spin channel (e.g., ref.174). It is questionable, however, whether this effect is important for the description of GMR in $3 d$-metal multilayers, because the short circuit effects, which appear if the intermixing at the interfaces is the only mechanism of scattering (see section 16), are avoided due to structural disorde and phonon scattering in real systems. The spin-orbit interaction might be important when heavy elements like gold or platinum are used as a spacer layer or introduced as impurities.
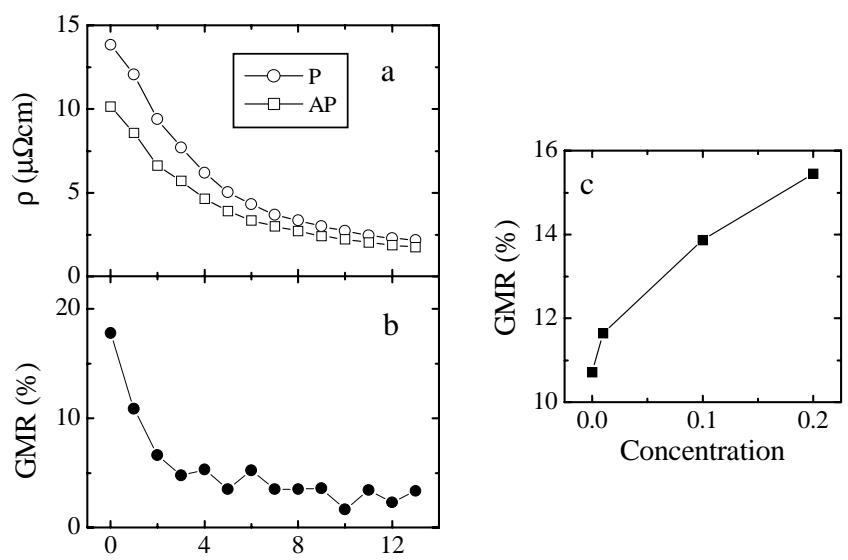

$\mathrm{Cu}$ thickness (ML)

Fig.38 Resistivity (a) and GMR (b) of a disorder-free $\mathrm{Co}_{3} / \mathrm{Cu}\left(d_{\mathrm{Cu}} / \mathrm{Co}_{3}(001)\right.$ trilayer embedded in semiinfinite $\mathrm{Cu}(001)$ substrates for parallel $(\mathrm{P})$ and antiparallel (AP) magnetizations as a function of the $\mathrm{Cu}$ layer thickness $d_{\mathrm{Cu}}$ and GMR (c) of a $\mathrm{Co}_{5} / \mathrm{Co}_{1-x} \mathrm{Cu}_{x} / \mathrm{Co}_{x} \mathrm{Cu}_{1-x} / \mathrm{Cu}_{7} /$ $\mathrm{Co}_{x} \mathrm{Cu}_{1-\mathrm{x}} / \mathrm{Co}_{1-\mathrm{x}} \mathrm{Cu}_{\mathrm{x}} / \mathrm{Co}_{6}(\mathrm{O01})$ trilayer versus concentration $x$ of interdiffused $\mathrm{Co}$ and $\mathrm{Cu}$ at the $\mathrm{Co} / \mathrm{Cu}$ interfaces. After Blaas et al.

The spin-orbit coupling is explicitly taken into account in a fully-relativistic calculation of GMR in $\mathrm{Co} / \mathrm{Cu}$ multilayers by Blaas et al. ${ }^{194}$ They use a screened KKR method adapted to layered system and a single-site coherent-potential approximation (CPA) to incorporate the effect of intermixing at the interfaces on electrical transport. Within their approach a magnetic multilayer is embedded between two semiinfinite substrates, which makes the system non-periodic in the direction 
perpendicular to the planes. The electrical conductivity of the multilayer is determined by summing the non-local conductivity $\sigma_{i j}$ only over the atomic layers of the imbedded system in accordance to equation (18.2). In this formulation the conductive substrates play the role of reservoirs, to where an electron will eventually leak out from the layered structure. Mathematically this implies that the Green's function, which describes the electronic properties of the multilayer and enters expression (18.3) for the non-local conductivity, has a self-energy contribution due to the semiinfinite substrates. Even when the multilayer is free of defects it exhibits resistivity, which is determined by the imaginary part of this self-energy. In this formulation the resistivity of the multilayer depends strongly on the electronic structure of the substrates and their coupling with the imbedded system. The resistivity comes therefore from the boundary conditions and decreases with the increasing thicknes of the disorder-free embedded film. ${ }^{194}$

Fig.38a shows the results of Blaas et al. ${ }^{194}$ for the resistivity of a $\mathrm{Co}_{3} / \mathrm{Cu}\left(d_{\mathrm{Cu}}\right) / \mathrm{Co}_{3}(001)$ trilayer embedded in semiinfinite $\mathrm{Cu}(001)$ substrates for parallel $(\mathrm{P})$ and antiparallel (AP) magnetizations as a function of the $\mathrm{Cu}$ layer thickness $d_{\mathrm{Cu}}$. No disorder was assumed in this calculation and the values of resistivity are entirely determined by the coupling with the Cu substrates. As is seen from Fig.38b, the magnitude of GMR decreases with increasing $\mathrm{Cu}$ layer thickness ranging from about $20 \%$ to about $2 \%$. These values increase within increasing Co layer thicknesses (not shown) as a result of the decreasing influence of the outer boundaries. Blaas et al. have also investigated the effect of intermixing within two monolayers of $\mathrm{Co}$ and $\mathrm{Cu}$ attached to the $\mathrm{Co} / \mathrm{Cu}$ interfaces. Fig.38c shows the intermixing within two monolayers of $\mathrm{Co}$ and $\mathrm{Cu}$ attached to the $\mathrm{Co} / \mathrm{Cu}$ interfaces. Fig. 38c shows the
results of their calculation for a $\mathrm{Co}_{5} / \mathrm{Co}_{1-\mathrm{x}} \mathrm{Cu}_{\mathrm{x}} / \mathrm{Co}_{\mathrm{x}} \mathrm{Cu}_{1-\mathrm{x}} / \mathrm{Cu}_{7} / \mathrm{Co}_{\mathrm{x}} \mathrm{Cu}_{1-\mathrm{x}} / \mathrm{Co}_{1-\mathrm{x}} \mathrm{Cu}_{\mathrm{x}} / \mathrm{Co}_{6}(001)$ trilayer as a function of concentration $x$ within the intermixed regions. As is seen from the figure, GMR increase with $x$, which reflects increasing spin-dependent scattering at the interfaces. Unlike refs.168, 170, 17 and 172, Blaas et al. ${ }^{194}$ find reasonable values of GMR due to the scattering at the intermixed interfaces. This is the consequence of the coupling to the attached $\mathrm{Cu}$ substrates, which leads to the broadening of electronic states of the $\mathrm{Co} / \mathrm{Cu} / \mathrm{Co}$ trilayer, thereby avoiding a short circuit current broadening of electronic states of the $\mathrm{Co} / \mathrm{Cu} / \mathrm{Co}$ trilayer, thereby avoiding a short circuit current
within the majority spin channel. It is not clear, however, what physical mechanism of scattering could provide the same broadening of states in the isolated multilayer as in the imbedded multilayer, in order to correlate the results of such computations with experiments. We also note that the increase in GMR as a function of the function contradicts the experiments of Suzuki and Taga ${ }^{73}$ who foun that the intermixing at the interfaces is detrimental for $\mathrm{GMR}$ in $\mathrm{Co} / \mathrm{Cu}$ multilayers. This is probably the result of misaligned magnetic moments and increasing structural disorder at the interfaces due to intermixing, which are not taken into account in the calculations of Blaas et al. ${ }^{194}$ Unfortunately Blass et al. do not discuss the effect of spin-orbit interaction, which makes their calculations differen from the non-relativistic formulations of the transport theory. The importance of the spin-orbi interaction in GMR of the $3 d$-metal magnetic multilayers remains elusive.

Spin mixing in the conductance can occur not only due to the spin-orbit coupling but also due to misaligned magnetic moments, which are present in magnetic layered systems due to interdiffusion at the interfaces. As was discussed in section 8 , experiments ${ }^{79}$ find that at room temperature there is a substantial reduction in the magnetization of permalloy near the interfaces with copper, which is equivalent to a magnetically-dead layer of $0.2 \mathrm{~nm}$ thickness. In order to investigate this effect, Oparin et $a l .{ }^{80}$ have performed calculations of the magnetic structure of the intermixed $\mathrm{Ni}_{80} \mathrm{Fe}_{20} / \mathrm{Cu}$ interfaces. They imbedded four atomic planes of $\mathrm{Ni}(111)$ in $\mathrm{Cu}$ and used the diffusion equation to calculate the configuration profile as a function of time. Assuming that $\mathrm{Cu}, \mathrm{Ni}$, and $\mathrm{Fe}$ have similar diffusion constants they replaced $\mathrm{Ni}$ by $\mathrm{Fe}$ at random in the $\mathrm{Cu} / \mathrm{Ni} / \mathrm{Cu}$ structure to arrive at the $\mathrm{Cu} / \mathrm{Ni}_{80} \mathrm{Fe}_{20} / \mathrm{Cu}$ structure. For a given atomic configuration the magnetic structure was calculated using the local scalable multiple scattering approach to local spin-density theory ${ }^{195}$ Fig. 39 shows the ground state magnetic configuration of the four layers of permalloy in a copper matrix afte interdiffusion has occurred. The $\mathrm{Fe}$ moments are about the same size as in pure $\mathrm{Ni}_{80} \mathrm{Fe}_{20}$, but their directions have become disordered. These misoriented moments can contribute to spin mixing an spin-flip scattering. Furthermore, the Ni moments are much smaller than for pure bulk $\mathrm{Ni}_{80} \mathrm{Fe}_{20}$ so the majority channel does not match nearly so well to Cu. Oparin $e t a l .^{80}$ conclude that the canting of the $\mathrm{Fe}$ moments and a reduction of the magnitude of the $\mathrm{Ni}$ moments at the interface is responsible for the measured "magnetically dead" layers ${ }^{79}$ and result in the strong reduction of GMR. Butler et al. ${ }^{196}$ found that placing a small amount of $\mathrm{Co}$ at the interfaces can dramatically increase the collinearity. The Fe moments become in much better alignment and the Ni moments become closer to their bulk values. This Co-induced improvement in collinearity is consistent with the enhancement of GMR obtained in experiments by Parkin ${ }^{77}$ (see also Fig.15)

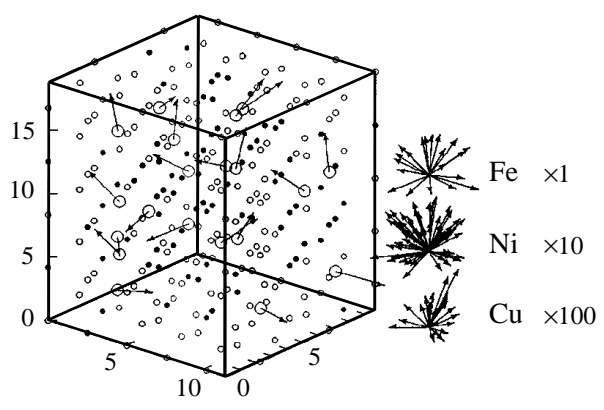

Fig.39 Atomic positions (in Å) and site magnetic moments (in arb.units) for a magnetic configuration of four atomic planes of $\mathrm{Ni}_{80} \mathrm{Fe}_{20}$ interdiffused into Cu. The small filled circles represent $\mathrm{Ni}$, the small open circles represent $\mathrm{Cu}$, and the large open circles represent Fe. All Fe moments, as well as Ni moments greater than $0.15 \mu_{\mathrm{B}}$ and Cu moments larger than $0.015 \mu_{\mathrm{B}}$ are depicted to the right-hand side with the indicated multiplying factors. After Oparin et al. ${ }^{80}$

\section{CPP GMR}

Since the successful demonstration of current-perpendicular-to-the-plane (CPP) magnetoresistance by Pratt $e t a l{ }^{13}$ this geometry has become the subject of much interest for studying GMR. The technique of using superconducting contacts ${ }^{13}$ produces uniform current density and has the advantage of relatively simple sample preparation. Unfortunately, measurements can be performed only at low temperatures. Other techniques, which avoid this problem, are based on lithographically defined pillar structures, ${ }^{14}$ on growing the magnetic multilayers on prestructured (grooved) substrates ${ }^{15,16}$ (Fig.3a), or on the electrodeposition of multilayer nanowires into the pores of an insulating polymer matrix ${ }^{17,18}$ (Fig $3 \mathrm{~b}$ ). These techniques have, however, disadvantages such as the difficulty to control the AP state, non-uniform currents in the lithographed samples, and the stronge difficulty to control the AP state, non-uniform currents in the lithographed samples, and the stronger
disorder in nanowires. Several reviews theoretical and experimental aspects of CPP GMR. In this section, we outline some results of these studies, referring the reader to the above reviews for a detailed discussion. We will, in particular, address novel issues, which have emerged recently. These have important consequences for our understanding of the physics of CPP GMR, as well as for interpreting the experimental data

Most experiments on CPP GMR are interpreted in terms of the series-resistor model, which was introduced in section 3. The series-resistor model is a better approximation for the CPP geometry than for the CIP geometry. This can be qualitatively understood by the following arguments. In the limit when the elastic mean free path $\lambda$ is short compared to the layer thicknesses, each layer can be 
considered as a separate resistor for the current flowing perpendicular to the planes of the multilayer In the opposite limit of a very long $\lambda$ the probability of scattering is the sum of the scattering probabilities within each layer. This implies that the conductivity is "self-averaging", leading to, like in the first case, resistors in series. This was first recognized by Zhang and Levy ${ }^{41}$ who have shown that for a multilayer which is described by a free-electron model with no potential steps at the interfaces, the conductivity of the multilayer for each spin channel can be expressed by the simple formula

$$
\sigma_{C P P}=\left(\frac{1}{L} \int_{L} \sigma^{-1}(z) d z\right)^{-1}=\frac{n e^{2}}{m}\left(\frac{1}{L} \int_{L} \Delta(z) d z\right)^{-1},
$$

where the local isotropic spin-dependent conductivity $\sigma(z)$ is given by expression (13.15) and $\Delta(z)$ is the local spin-dependent scattering rate. This formula is identical to that within the series-resistor model, in which the resistivity of the multilayer is determined by resistivities of the individual layers $\rho_{i}$ and their thicknesses $d_{i}$

$$
\rho_{C P P}=\frac{1}{L} \int_{L} \rho(z) d z=\frac{1}{L} \sum_{i} d_{i} \rho_{i},
$$

where $i$ is an index of the layer. According to Zhang and Levy ${ }^{41}$ and Camblong $e t ~ a l .{ }^{43}$ the mean-free path is not a relevant scale for CPP GMR. Indeed, although the layer- and spin-dependent resistivitie $\rho_{i}=\frac{m \Delta_{i}}{n e^{2}}=\frac{k_{F}}{n e^{2} \lambda_{i}}$ in formula (VI.2) depend on the mean free paths $\lambda_{i}$, the CPP GMR is determined only by the layer thicknesses. For example, as we saw in section 3 , the resistor model predicts that GMR varies as $1 / d_{N M}^{2}$ for large spacer layer thicknesses $d_{N M}$. This is in contrast to the CIP geometry, in which for large $d_{N M}$ GMR scales exponentially, i.e. as $\exp \left(-d_{N M} / \lambda_{N M}\right)$, with the mean free path in the non-magnetic spacer layer $\lambda_{N M}$ (see section 12). We stress, however, that the conclusion about the irrelevance of the mean free path and the applicability of the series-resistor model to CPP GMR is inherent to the free-electron model with no potential steps at the interfaces. As we will see below, the mean free path can play an important role in CPP GMR.

The quantity which is measured in the CPP geometry is the conductance per unit area, or equivalently its inverse, the resistance $A R$, where $A$ is the area through which the current flows. The CPP GMR is defined by the ratio $\left(A R_{A P}-A R_{P}\right) / A R_{P}=A \Delta R /\left(A R_{A P}-A \Delta R\right)$. These quantities can be expressed in terms of the series-resistor model, which was elaborated by Lee et al. ${ }^{197}$ to include spindependent bulk resistivities, spin-dependent interface resistances, and contact resistances due to superconducting leads. According to their model the resistances $A R_{A P}$ and $A \Delta R$ of the multilayer with the number of bilayers $N$ are given by

$$
\begin{aligned}
& A R_{A P}=2 A R_{S / F M}+2 N \rho_{N M} d_{N M}+N \rho_{F M}^{*} d_{F M}+2 N A R_{F M / N M}^{*}, \\
& A \Delta R=N^{2}\left(\beta \rho_{F M}^{*} d_{F M}+2 \gamma A R_{F M / N M}^{*}\right)^{2} / A R_{A P} .
\end{aligned}
$$

They are determined by six parameters, namely the resistance of the superconductor/ferromagnet contact $A R_{S / F M}$, the resistivity of the nonmagnetic layer $\rho_{N M}$, the renormalized resistivity of the ferromagnetic layer $\rho_{F M}^{*}=\rho_{F M} /\left(1-\beta^{2}\right)$, the renormalized interface resistance $A R_{F M / N M}^{*}=A R_{F M / N M} /\left(1-\gamma^{2}\right)$, and the scattering spin-asymmetry parameters for the bulk, $\beta$, and for the interface, $\gamma$. Parameter $\beta$ is related to the spin asymmetry of the bulk scattering within the ferromagnetic layer $\alpha_{F M}=\rho_{F M}^{\downarrow} / \rho_{F M}^{\uparrow}$, which was introduced in section 3, by the expression $\alpha_{F M}=(1+\beta) /(1-\beta)$. Parameter $\gamma$ is related to the spin asymmetry of the interface scattering at the
FM/NM interfaces $\alpha_{F M / N M}=R_{F M / N M}^{\downarrow} / R_{F M / N M}^{\uparrow}$ by the expression $\alpha_{F M / N M}=(1+\gamma) /(1-\gamma)$. The values of $A R_{S / F M}, \rho_{N M}$, and $\rho_{F M}$ can be determined independently from experiments on $S / F M\left(d_{F M}\right) / S$, $S / N M\left(d_{N M}\right) / S$ trilayers. $A R_{S / F M}$ can be found from the ordinate intercept of a linear fit to AR versus $d_{F M}$ for a series of $S / F M\left(d_{F M}\right) / S$ trilayers. The resistivities $\rho_{N M}$, and $\rho_{F M}$ are determined by the slope of linear-fit curves 'resistance versus thickness'. This reduces the number of adjustable parameters to only three, namely $A R_{F M / N M}^{*}, \beta$ and $\gamma$. These parameters can be found experimentally ${ }^{198}$ by measuring $A R_{A P}$ and $A \Delta R$ for samples with varying $d_{F M}$ and $d_{N M}$ and using equations (VI.3) and (VI.4).

Table 1 summarizes the results obtained for $\mathrm{Co} / \mathrm{Cu}$ multilayers by different groups using various techniques. The first and second columns display the parameters obtained from experiments on multilayers fabricated by sputtering ${ }^{24}$ and $\mathrm{MBE}^{199}$ using superconducting contacts. The third column shows the parameters derived from fitting the experimental data on multilayers grown on groove shows the parameters derived from fitting the experimental data on multilayers grown on grooved
substrates. ${ }^{96}$ The fourth and fifth columns display the parameters obtained from measurements on substrates. ${ }^{96}$ The fourth and fifth columns display the parameters obtained from measurements on
multilayered nanowires prepared by electrodeposition. ${ }^{200,201}$ As is seen from the table, different fabrication techniques result in quite different bulk resistivities of $\mathrm{Cu}$ and $\mathrm{Co}$ in the $\mathrm{Co} / \mathrm{Cu}$ multilayer reflecting a variation in the defect density. This leads to a sizable spread in the values of the scattering spin-asymmetry parameters $\beta$ and $\gamma$. However, despite this spread, it is evident from Table 1 that the spin asymmetry of scattering at the interface, $\gamma$, is always higher than the spin asymmetry of scattering in bulk $\mathrm{Co}, \beta$, whatever the method of preparation of the $\mathrm{Co} / \mathrm{Cu}$ multilayers is. This fact demonstrates the decisive role of the spin-dependent interface scattering for CPP GMR in the $\mathrm{Co} / \mathrm{Cu}$ multilayers. Indeed, according to formula (VI.4), the contribution from the bulk resistance becomes equal to the contribution from the interface resistance when the FM layer thickness is $d_{F M}=2 \gamma A R_{F M / N M}^{*} / \beta \rho_{F M}^{*}$. Using the parameters from ref.24 (the first column in Table1) this gives $d_{F M} \sim 20 \mathrm{~nm}$. We see therefore, that for FM layer thicknesses of a few $\mathrm{nm}$ the dominant contribution to GMR comes from the spin-dependent interface resistance.

TABLE 1

Spin-dependent scattering parameters obtained from CPP GMR experiments on $\mathrm{Cu} / \mathrm{Co}$ multilayers at low temperatures using the series-resistor model. Measurements are performed by various techniques as indicated in the table. The parameters are determined from the resistances at the saturation field and the field of the local resistance maximum after saturation.

\begin{tabular}{lccccc}
\hline & $\begin{array}{c}\text { ref.24 } \\
\text { multilayers } \\
\text { sputtering } \\
4.2 \mathrm{~K}\end{array}$ & $\begin{array}{c}\text { ref.199 } \\
\text { multilayers } \\
\text { MBE } \\
4.2 \mathrm{~K}\end{array}$ & $\begin{array}{c}\text { ref.96 } \\
\text { grooved substr. } \\
\text { MBE } \\
4.2 \mathrm{~K}\end{array}$ & $\begin{array}{c}\text { ref.200 } \\
\text { nanowires } \\
\text { electrodep. } \\
77 \mathrm{~K}\end{array}$ & $\begin{array}{c}\text { ref.201 } \\
\text { nanowires } \\
\text { electrodep. } \\
20 \mathrm{~K}\end{array}$ \\
\hline \hline & & & & & \\
$\rho_{C u}(\mu \Omega \mathrm{cm})$ & $0.6 \pm 0.1$ & $1.3 \pm 0.3$ & $0.39 \pm 0.07$ & 3.1 & $1.3-3.3$ \\
$\rho_{C o}^{*}(\mu \Omega \mathrm{cm})$ & $6.6 \pm 0.5$ & $3.0 \pm 0.6$ & $4.2 \pm 0.7$ & $18 \pm 2$ & $51-57$ \\
$\beta$ & $0.38 \pm 0.06$ & $0.48 \pm 0.04$ & $0.17 \pm 0.03$ & $0.36 \pm 0.04$ & $0.46 \pm 0.05$ \\
$A R_{C o / C u}^{*}\left(\mathrm{f} \Omega \mathrm{m}^{2}\right)$ & $0.38 \pm 0.03$ & $0.43 \pm 0.04$ & $0.25 \pm 0.04$ & $0.30 \pm 0.05$ & $0.3-1.1$ \\
$\gamma$ & $0.71 \pm 0.05$ & $0.71 \pm 0.02$ & $0.45 \pm 0.09$ & $0.85 \pm 0.10$ & $0.55 \pm 0.07$ \\
\hline
\end{tabular}

It is known that there are two contributions to the interface resistance: ${ }^{202}$ the first one comes from the potential step between the ferromagnetic and nonmagnetic metal layers and the second one results 
from interface disorder. Zhang and Levy ${ }^{203}$ have shown that these two contributions are not additive and that diffuse scattering by the interface disorder may either enhance or reduce the interface resistance, the latter occurring for large reflection coefficients. These free-electron considerations in the presence of the intrinsic multilayer potential ${ }^{202,203}$ contain unknown parameters such as the spindependent potential steps. The interface resistance can, however, be modeled from first principles. there is no coherent transmission between adjacent interfaces. The latter approximation implies complete diffuse scattering in the bulk and justifies the series-resistor model. For the (111)-oriented multilayer they found the values of the interface resistance, $A R_{C o / C u}^{*}=0.44 \mathrm{f} \Omega \mathrm{m}^{2}$, and the spin asymmetry parameter, $\gamma=0.54$, which are in reasonable agreement with the experimental values presented in Table 1. Schep et al. concluded that the experimentally observed interface resistance in the $\mathrm{Co} / \mathrm{Cu}$ multilayers can be explained largely in terms of specular scattering at the $\mathrm{Co} / \mathrm{Cu}$ interfaces. A similar conclusion was arrived at by Chen et al. ${ }^{205}$ who used a multiband tight-binding model to calculate the $\mathrm{Co} / \mathrm{Cu}$ interface resistance and the scattering spin-asymmetry parameter $\gamma$ in the presence of the spin-dependent or spin-independent impurity potential at the interfaces. The importance of specular scattering at the $\mathrm{Co} / \mathrm{Cu}$ interface was also confirmed in calculations by Stiles and Penn, ${ }^{206}$ who argued that the measured interface resistances might not be dominated by defect scattering as is often assumed.

The interpretation of the experimental data within the series-resistor model assumes that the interfaces can be uniquely described using two parameters, $A R_{F M / N M}^{*}$ and $\gamma$, independent of the layer thickness separating them. Schep et $a l^{204}$ found, however, that the $\mathrm{Co} / \mathrm{Cu}$ interface resistance is strongly reduced if the bulk propagation is ballistic rather than diffusive. This implies that the interface resistance can be different for thin and thick layers between the interfaces making, in general, the series resistor-model inapplicable.

The fact that the interface resistance can be dependent on the layer thickness has been explicitly demonstrated by model calculations of Tsymbal and Pettifor. ${ }^{207}$ They used a simple single-band tightbinding model and calculated the resistance of a disordered metal layer placed between two perfect leads using the Kubo formula and the recursive approach, as is described in section 14. Fig. 40a demonstrates the results of these computations. The open squares show the resistance of the metal-1 layer, which is characterized by the on-site atomic energy $E_{1}=3$ (in units of the hopping integral), as a function of its thickness $d$ when it is placed between perfect leads of the same metal. The linear variation seen is evidence of the Ohmic regime of conductance, the slope of the curve determining the resistivity of disordered metal 1 and the ordinate intercept determining the resistance $A R_{1}$ of disorderfree metal 1 . When a lead of metal 2 with a different on-site atomic energy $E_{2}=5$ is attached to the disordered metal 1 layer, an interface resistance is introduced due to the potential step at this interface. As is seen from the open circles in Fig.40a, this shifts the curve up, the shape of the curve remaining linear with the same slope. The magnitude of this shift equals to $A R_{\text {int }}+\left(A R_{1}+A R_{2}\right) / 2-A R_{1}$, where $A R_{\text {in }}$ is the interface resistance and $A R_{1}$ and $A R_{2}$ are the resistances of the infinite disorder-free leads of metal 1 and metal 2 respectively. ${ }^{204}$ Attaching a second lead of metal 2 to the disordered layer of metal 1 does not, however, result in a rigid upward shift of the curve as it would be expected due to the additional interface resistance within the series-resistor model. As is evident from the full circles in Fig.40a, the resistance becomes a non-linear function of the metal thickness. This behavior is a consequence of the quantum-well bound states, which are created in the metal 1 layer, when it is placed between the leads of a higher electronic potential. These bound states reduce the number of conducting channels in this layer, which leads to the reduced interface resistance and the enhanced effective resistivity of the metal. With increasing thickness of the disordered metal layer, defect scattering redistributes the current-carrying electrons between the various conducting channels, thereby enhancing the interface resistance, which is plotted in Fig.40b. The characteristic length scale for this change is the mean free path, which is estimated as $\lambda \approx 25 a$ in this calculation. When the layer thickness becomes much larger than the mean free path the series-resistor behavior is recovered, which is reflected in a linear variation of the resistance at $d>100 a$ in Fig.40a and the saturation of the interface resistance in Fig.40b. An interface resistance that depends on the layer thickness is a feature of perpendicular transport, which lies beyond the series-resistor model and makes the mean free path an important parameter for CPP GMR. We note that noticeable oscillations in the resistance at very small thicknesses, which are seen from the full circles in Fig.40, are reminiscent of the oscillations in the ballistic regime of conduction. ${ }^{164}$

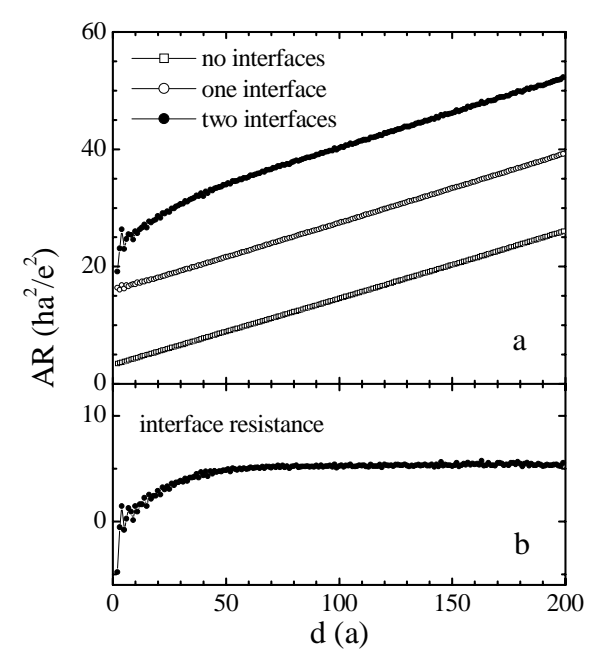

Fig.40 (a): Resistance of a disordered metal-1 layer versus its thickness $d$ (in units of the lattice parameter $a$ ) when it is placed between the perfect leads of the same metal 1 (open squares), between the left lead of metal 2 and the right lead of metal 1 (open circles), and between the leads of metal 2 (full circles). (b): Layer-thickness-dependent interface resistance, which is calculated by taking the difference between the curves corresponding to one and two interfaces in figure (a) and, then, by substrating the average resistance, $\left(A R_{1}+A R_{2}\right) / 2$, of the infinite disorder-free leads of metal 1 and metal 2. In the calculations the metals differ by the on-site atomic energies which are equal to $E_{1}=3$ and $E_{2}=5$ respectively. The energies are measured in units of the hopping integral relative to the Fermi energy which lies at zero. Bulk disorder is introduced as a random variation of the on-site atomic energies with a uniform distribution of standard deviation $\gamma=0.5$. The calculated conductance is averaged over 80 random configurations. The size of the system is extended to infinity in the direction perpendicular to the current by introducing a unit cell of $16 \times 16$ atoms in the transverse direction and imposing periodic boundary conditions. After Tsymbal and Pettifor. ${ }^{2}$

A realistic band structure of the multilayer predicts a more diverse pattern of behavior for the layer-thickness-dependent interface resistance. At a small layer thickness separating two interfaces the interface resistance can be enhanced or reduced depending on the band structure of adjacent metals. This was illustrated by Tsymbal, ${ }^{208}$ who calculated the resistance and CPP GMR for the $\mathrm{Co}_{10} / \mathrm{Cu}(d) / \mathrm{Co}_{10}$ trilayer versus $\mathrm{Cu}$ layer thickness $d$. As is evident from Fig.41a, the resistance of the majority-spin electrons within the $\mathrm{P}$ configuration (the open circles) and the resistance for the AP 
configuration (the diamonds) show pronounced non-linearity as a function of the $\mathrm{Cu}$ thickness. For comparison the solid lines in Fig.41 show the resistance of bulk $\mathrm{Cu}$, which was offset to fit the respective resistances of the $\mathrm{Co}_{10} / \mathrm{Cu}(d) / \mathrm{Co}_{10}$ trilayer at large $\mathrm{Cu}$ thicknesses. A sizeable deviation of the symbols from the solid lines is seen in Fig.2a at small $d$, which implies that the $\mathrm{Co} / \mathrm{Cu}$ interface resistance depends on on the $\mathrm{Cu}$ layer thickness. This difference has opposite sign for the $\mathrm{P}$ and AP configurations. In the case of $\mathrm{P}$ magnetizations, the interface resistance increases with the $\mathrm{Cu}$ layer thickness for both the majority- and minority-spin electrons (the open circles and squares in Fig.41a), which is due to the bound states within the $\mathrm{Cu}$ layer placed between the two Co layers. It is interesting that the minority electrons display a less-pronounced departure from linearity. This is due to the effect of disorder, which intermixes the closely lying Co $d$ bands, thereby smearing out the potential well Indeed, a separate calculation, in which no disorder was assumed within the Co layers, demonstrates much stronger non-linearity for the minority $\mathrm{Co}_{10} / \mathrm{Cu}(d) / \mathrm{Co}_{10}$ resistance (not shown). The situation is, however, different for the AP configuration. As is evident from the diamonds in Fig.41a, the interface resistance decreases with the Cu layer thickness. The effect arises from the very distinct electronic structures of the majority and minority bands in Co. In this case, scattering by disorder in the $\mathrm{Cu}$ layer assists the electrons which have passed the first $\mathrm{Co} / \mathrm{Cu}$ interface to be transmitted acros the second $\mathrm{Cu} / \mathrm{Co}$ interface, reducing the interface resistance with increasing the $\mathrm{Cu}$ layer thickness.

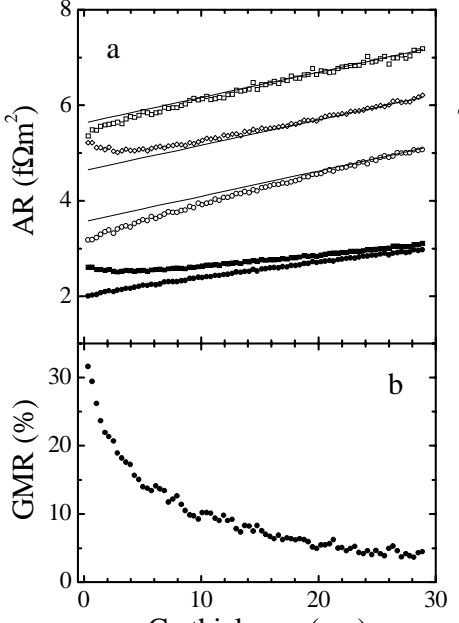

Cu thickness (nm)

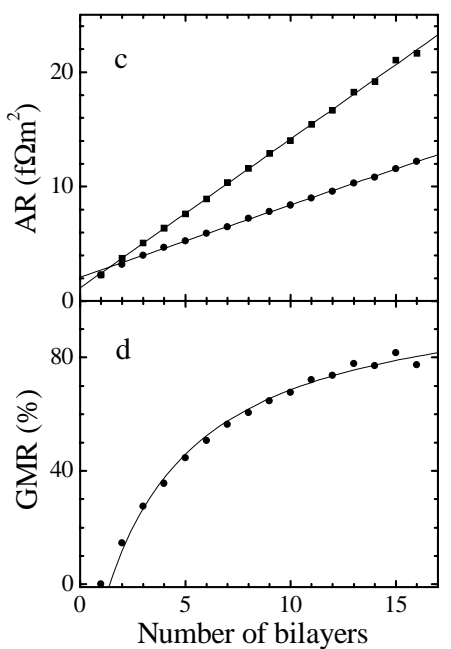

Number of bilayers
Fig.41 Resistance (a) and GMR (b) of the $\mathrm{Co}_{10} / \mathrm{Cu}(d) / \mathrm{Co}_{10}$ trilayer as a function of the $\mathrm{Cu}$ layer thickness $d$, and resistance (c) and GMR (d) of the $\left(\mathrm{Cu}_{10} / \mathrm{Co}_{10}\right)_{\mathrm{N}}$ multilayer as a function of the number of $\mathrm{Co} / \mathrm{Cu}$ bilayers $N$. Open symbols show the resistance per spin of the majority (circles) and minority (squares) electrons for the P configuration and for the AP configuration (diamonds). In panel (a) the full symbols show the total resistance for the $\mathrm{P}$ (circles) and AP (squares) configurations and the solid lines display the bulk $\mathrm{Cu}$ resistance which was offset to fit the respective resistances for the trilayer at large Cu thicknesses. The disorder parameter is set equal to $\gamma=0.6 \mathrm{eV}$, which gives a bulk resistivity of $4.6 \mu \Omega \mathrm{cm}$ for $\mathrm{Cu}$ and $14.3 \mu \Omega \mathrm{cm}$ for
Co. Solid lines in calculated from this fit. After Tsymbal. ${ }^{20}$
The dependence of the interface resistance on the layer thickness makes the $\mathrm{Co} / \mathrm{Cu}$ interface resistance different for the $\mathrm{P}$ and $\mathrm{AP}$ configurations. This effects the variation of the resistance and GMR as a function of the number of the $\mathrm{Co} / \mathrm{Cu}$ bilayers $N$ in the $(\mathrm{Cu} / \mathrm{Co})_{\mathrm{N}}$ multilayer, which is shown in Figs.41c,d. ${ }^{208}$ As is evident from the fit displayed in Fig.4a by the solid lines, the P and AP resistances increase in a nearly linear fashion as a function of $N$. This is exactly what one could expect from the series-resistor model. The reality is, however, more complex. The extrapolation of the linea fit to zero $N$ results in different resistances for the $\mathrm{P}$ and AP magnetizations. For the $\mathrm{P}$ configuration we obtain $3.5 \mathrm{f} \Omega \mathrm{m}^{2}$, which is by a factor of two higher than the value for the AP configuration of $1.8 \mathrm{f} \Omega \mathrm{m}^{2}$. This enhanced value is a consequence of the thickness-dependent interface resistance. For the majority-spin electrons, which predominantly contribute to the resistance within the $\mathrm{P}$ configuration, the interface resistance is reduced for the small number of the $\mathrm{Co} / \mathrm{Cu}$ bilayers. Although the thickness-dependent interface resistance saturates very quickly (almost within two-three repeats), it results in the offset of the resistance-curve origin between the $\mathrm{P}$ and AP configurations, as can be seen from Fig.41c. This leads to a very slow saturation in the CPP GMR, i.e. $\propto 1 / N$, which is evident from Fig.41d.

The fact that the interface resistance depends on the proximity of the other interfaces and bulk scattering rates is in agreement with the prediction of Butler et al. ${ }^{209}$ who solved the Boltzmann equation in the CPP geometry including the scattering-in term and assuming that the electronic structure is different for different layers. They found that the interface resistance is affected by the exponential terms in the electrochemical potential that decays at a rate comparable to the mean free path. In the limit of uniform band structure they recovered the solution of the series-resistor model The dependence of the interface resistance on the number of interfaces was found in recent calculations by Xia et al. ${ }^{210}$ They modeled interface disorder using large lateral supercells within the TB-LMTO method, ${ }^{211}$ which allowed them to treat specular and diffuse scattering on an equal footing. They demonstrated that in $\mathrm{Co} / \mathrm{Cu}$ multilayers with $10 \mathrm{ML}$ layer thicknesses and intermixed interfaces the $\mathrm{Co} / \mathrm{Cu}$ interface resistance decreases for both the majority- and minority-spin electron with increasing number of bilayers. Very recently Shpiro and Levy ${ }^{212}$ have investigated the form of the chemical potential in the presence of the diffuse and specular scattering at the interface between two semiinfinite metals by solving the linearized Boltzmann equation within a free-electron model assuming a potential step at the interface. They found that the interface resistance deduced from the voltage drop measured far from the interface is different from the interface resistance that would be obtained by measuring the voltage drop directly at the interface. They concluded that the interface resistance is dependent on the resistance in the adjacent layers and that one has to re-examine the parameters obtained from the analysis of CPP transport data on metallic multilayers that have been modeled by the series-resistor model.

Experimental evidence for the breaking down of the series-resistor model and the relevance of the mean free path to CPP GMR have recently been presented by Bozec et al. ${ }^{213}$ They carried out an experimental study of the magnetoresistance $R(H)$ in the CPP geometry for two types of magnetic multilayers, interleaved and separated, that differ only in the ordering of the magnetic layers: $[\mathrm{Co}(1 \mathrm{~nm}) / \mathrm{Cu}(20 \mathrm{~nm}) / \mathrm{Co}(6 \mathrm{~nm}) / \mathrm{Cu}(20 \mathrm{~nm})]_{\mathrm{N}}$ and $\left.[\mathrm{Co}(1 \mathrm{~nm}) / \mathrm{Cu}(20 \mathrm{~nm})]_{\mathrm{N}} \mathrm{Co}(6 \mathrm{~nm}) / \mathrm{Cu}(20 \mathrm{~nm})\right]_{\mathrm{N}}$. Due to the different thicknesses of the Co layers, $1 \mathrm{~nm}$ and $6 \mathrm{~nm}$ respectively, these layers had differen coercivities which resulted in the magnetization layer switching at different magnetic fields. The series resistor model predicts that in the CPP mode $R(H)$ is independent of the ordering of the layers. Nevertheless, as is seen from Fig.42a, the measured $R(H)$ curves are found to be completely differen for the two cases. The maximum value of $R(H)$ is much larger for the interleaved configuration than for the separated configuration. It was also found that the difference in GMR between the interleaved and separated configurations increases with increasing number of repeats $N$.

Calculations based on a realistic band structure and the Kubo formula show that the results of these experiments are the consequence of a long mean free path. ${ }^{213}$ Fig. $42 \mathrm{~b}$ displays the calculated resistance versus thickness of the $\mathrm{Co} / \mathrm{Cu}$ multilayer: the rightmost points in this figure determine the 
resistance of the full $\left(\mathrm{Co}_{10} / \mathrm{Cu}_{10}\right)_{4}$ structure. As is seen, the result of the calculations is in agreemen with the experiments: GMR for the interleaved configuration is higher than GMR for the separated configuration. There are a few important points, which follow from this modeling. (i) With increasing disorder in the $\mathrm{Co} / \mathrm{Cu}$ multilayer the difference in GMR between the interleaved and separated configurations becomes smaller and eventually disappears when the mean free path becomes much less than the layer thicknesses. This is due to the fact that in this limit the series resistor mode becomes justified. (ii) There is no difference in GMR between the interleaved and separated configurations within a single band tight-binding model with spin- and layer-dependent disorder, which determines different scattering rates for the majority- and minority-spin channels and for various layers. This is in agreement with the models, which are based on a free-electron ban structure for all layers. ${ }^{41-43}$ (iii) The calculation reproduces the important feature of the experiments, namely, an increase in the difference GMR between the interleaved and separated configurations with increasing the number of the $\mathrm{Co} / \mathrm{Cu}$ bilayers.

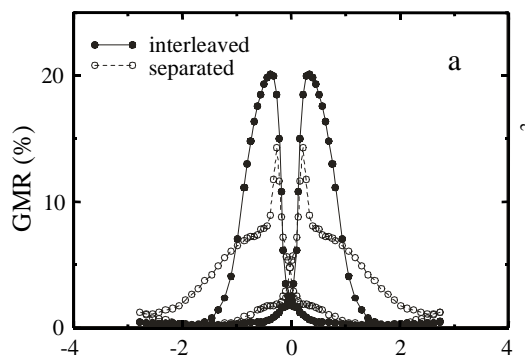

$\mathrm{H}(\mathrm{kG})$

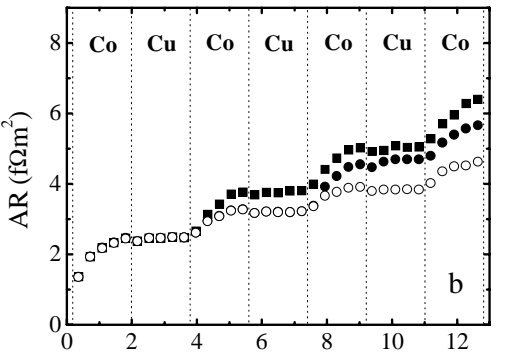

Thickness (nm)
Fig.42 (a): Experimental magnetic-field dependence of GMR for the interleaved $[\mathrm{Co}(1 \mathrm{~nm}) / \mathrm{Cu}(20 \mathrm{~nm}) / \mathrm{Co}(6 \mathrm{~nm}) / \mathrm{Cu}(20 \mathrm{~nm})]_{4}$ multilayer (squares) and the separated $\left.[\mathrm{Co}(1 \mathrm{~nm}) / \mathrm{Cu}(20 \mathrm{~nm})]_{4} \mathrm{Co}(6 \mathrm{~nm}) / \mathrm{Cu}(20 \mathrm{~nm})\right]_{4}$ multilayer (circles). (b): Calculated resistance of the $\mathrm{Co} / \mathrm{Cu}$ multilayer which contains $10 \mathrm{ML}$ of $\mathrm{Co}$ and $10 \mathrm{ML}$ of $\mathrm{Cu}$ within each layer as a function of the total thickness of the multilayer for the parallel magnetizations (open circles) and for the antiparallel magnetizations (full symbols) within the interleaved (squares) and separated (circles) configurations. After Bozec et al. ${ }^{213}$

These results have been very recently confirmed by Kudrnovsky et al. ${ }^{214}$ who used an efficien method for the first-principle computations of CPP GMR developed earlier. ${ }^{211}$ The method is based on the recursive technique, which is implemented within the TB-LMTO method, and allows treating various types of disorder by using a lateral supercell confined to individual atomic layers. The sensitivity of the GMR ratio to the order of the magnetic layers was also found in the calculations by Sanvito et al. $^{215}$ within a simple tight-binding two-band model

An interesting prediction has been made by Mathon ${ }^{216}$ who found an exponential growth of CPP GMR in magnetic multilayers with randomly varying thickness of the layers, which may reflect the island-like growth in epitaxial heterostructures. The predicted effect arises due to the localization of the electrons in both spin channels within the antiparallel configuration of the multilayer. The localization appears, however, as a result of disregarding other types of disorder in the multilayer. This assumption makes the system quasi one-dimensional with a random potential which localizes the wave function at the distance of the order of $l_{l o c} \sim N \lambda$, where $\lambda$ is the mean free path and $N$ is the number of open conducting channels in the one-dimensional system. The predicted large values of GMR will, therefore, be destroyed in real multilayers. The disorder-induced enhancement of GMR was recently found by Sanvito et al. ${ }^{217}$ However, as was shown in ref.208, this enhancement is due to the small size of the unit cell in the transverse direction of the multilayer which was used in these computations.

All the above models assume that there is no spin-flip scattering and, therefore, the electrical current can be described independently within the two spin-conduction channels. In bulk $3 d$ metals the probability of spin-flip scattering processes is normally small as compared to the probability of the scattering processes in which the spin is conserved such as electron-phonon scattering. It is only at temperatures comparable to the Curie temperature that spin-flip scattering processes due to electron-magnon interactions cause mixing of the electrons within the two spin channels. This means that spin is conserved over long distances and, therefore, the electrical conduction occurs in paralle for the two spin channels. These arguments allowed us to ignore spin-flip scattering when considering the CIP geometry, because CIP GMR is exponentially dependent on the mean free path, which is normally much shorter than the spin-diffusion length.

In the CPP geometry the situation is different. In the limit of large layer thicknesses the seriesresistor model predicts a constant GMR. This can be seen from equations (VI.3) and (VI.4): at large $d=d_{N M}=d_{F M}$ the contribution from the interface resistances can be neglected and both $A R_{A P}$ and $A \Delta R$ become proportional to $d$, which implies that CPP GMR is independent of the layer thickness. As has been shown by Valet and Fert, ${ }^{218}$ this is not the case when a finite spin-diffusion length is taken into account. The model of Valet and Fert is based on a solution of the Boltzmann equation within a freeelectron model, which in addition to the usual electron relaxation term for the momentum takes into account the spin relaxation term due to spin-flip scattering. The spin relaxation balances spin accumulation, which is generated when the electrical current flows perpendicular to the interfaces due to the spin splitting of the chemical potential. ${ }^{218}$ The spin-flip scattering determines the spin-diffusion length - a scale that controls the distance over which Mott's two-current model holds. The model of Valet and Fert predicts that at large layer thicknesses CPP GMR decays exponentially, the length scale of this decay being determined by the spin diffusion length. In the limit when the layer thicknesses are much shorter than spin-diffusion length, this model reduces to the series-resisto model, ${ }^{197}$ in which the resistances of the multilayer are described by equations (VI.3) and (VI.4).

The model of Valet and Fert has been extensively used to treat the experimental data on CPP GMR and to extract the values of the spin-diffusion length in various ferromagnetic and non-magnetic metals and their alloys. In particular, Yang et al. ${ }^{219}$ have shown that the spin-diffusion length in $\mathrm{Cu}$ and $\mathrm{Ag}$ can be dramatically shortened by doping these metals with Pt and Mn impurities. This shortening of the spin-diffusion length was ascribed to the spin-flip scattering associated with the spin-orbit coupling at Pt sites and with the spin-spin coupling at Mn sites. An unexpectedly low spindiffusion length was found for permalloy in experiments on $\mathrm{Ni}_{80} \mathrm{Fe}_{20} / \mathrm{Cu} / \mathrm{Ni}_{80} \mathrm{Fe}_{20}$ exchange-biased spin valves using superconducting contacts ${ }^{220}$ and in experiments on electrodeposited $\mathrm{Ni}_{80} \mathrm{Fe}_{20} / \mathrm{Cu}$ multilayered nanowires. ${ }^{221}$ We refer the reader to the reviews ${ }^{22,24,28}$ and original papers cited in these reviews for a detailed discussion of these and other studies of the spin diffusion length in various metals. Here we would like to note that these investigations do not take into account the influence of the finite-size effects on the interface resistance described above. The departures from the seriesresistor model is normally interpreted in terms of the contributions from spin-flip scattering either in ferromagnetic or in non-magnetic metals. For example, Park et al. ${ }^{222}$ investigated the influence of the inserted metal $\mathrm{M}=\mathrm{Fe}_{50} \mathrm{Mn}_{50}, \mathrm{~V}, \mathrm{Nb}$, and $\mathrm{W}$ on $\mathrm{CPP}$ GMR in $\mathrm{Fe}_{50} \mathrm{Mn}_{50} / \mathrm{Ni}_{80} \mathrm{Fe}_{20} /(\mathrm{Cu} / \mathrm{M}(d) / \mathrm{Cu})_{\mathrm{N}} /$ $\mathrm{Ni}_{80} \mathrm{Fe}_{20}$ spin valves and interpreted the observed exponential variation of $A \triangle R=A R_{A P}-A R_{P}$ as a function of the metal layer thickness $d$ in terms of the spin memory loss in the bulk of these metal and at the $\mathrm{Cu} / \mathrm{M}$ interfaces. This variation might, however, be caused by the layer-thicknessdependent interface resistance, which approaches its asymptotic value exponentially, scaling with the mean free path (see Fig.40b). In order to clarify this matter it would be very useful to compare the results of such or similar experiments with accurate calculations of CPP GMR based on realistic band structures. 


\section{CONCLUSIONS}

Giant magnetoresistance is the large change in electrical resistance of metallic layered system when the magnetizations of the ferromagnetic layers are reoriented relative to one another under the application of an external magnetic field. This reorientation of the magnetic moments alters both the electronic structure and the scattering of the conduction electrons in these systems, which causes the change in the resistance. Various types of magnetic layered structures have been found which show sizable values of GMR. Highest values are obtained in magnetic multilayer structures, such as $\mathrm{Fe} / \mathrm{C}$ and $\mathrm{Co} / \mathrm{Cu}$, which remain attractive from the point of view of studying the fundamental physic involved. The exchange-biased spin valves show a combination of properties that make these systems more useful for applications in low-field sensors, such as read heads for magnetic recording.

The discovery of GMR has stimulated significant progress in the theory of electronic transport in magnetic layered structures. First theories were based on simplified band structures, such as freeelectron models or single-band tight-binding models. These models are physically transparent and though simple, capture some important physics of GMR. For example, a free-electron theory based on the semiclassical description of electronic transport provided an understanding of the thicknes dependence of CIP GMR in magnetic multilayers and spin valves. However, these simple ban incorporating an accurate band structure of the multilayer within the theory of GMR is crucial. Another important ingredient for the predictive modeling of the conductivity and magnetoresistance in real metallic layered structures is using a quantum-mechanical theory of transport. The semiclassical Boltzmann transport theory can only be applied in systems with sufficiently low defect/impurity density. It breaks down in magnetic multilayers of practical interest because the the quantum-mechanical theory within an accurate multiband treatment of the electronic structure is the only way to make definitive statements about the origins of GMR.

The principal challenge for first-principle modeling lies in the realistic description of the defect scattering. A reliable approach must include contribution to the resistivity from intrinsic structura defects, which are always present in experimental conditions. However, proper first-principle treatment of all the existing defects in the multilayers is very complicated and, therefore, reliable simplified models become of great importance. The presence of structural defects may also be decisive for predicting realistic values of the scattering spin asymmetries of impurities, since some results obtained in the dilute limit in the absence of structural defects disagree strongly with experiment. Including misaligned magnetic moments at the interfaces in the transport theory is an important issue, which has not yet been considered in detail. Progress in developing a fully relativistic theory of transport may help to elucidate the influence of spin-orbit scattering on GMR and to calculate the spin-diffusion length in those cases when it originates from the spin-orbit interaction at some impurities. Considering the effect of the electron-phonon scattering is important for description of the temperature dependence of GMR. Little has been done to include this mechanism of scattering in the theory to date. The problem is, however, very complicated - it is already difficult to calculate the temperature dependence of the resistivity of bulk non-magnetic metals.

Recent experimental and theoretical results indicate that the mechanism of GMR within the CPP geometry has not yet been fully understood. It has been believed for a long time that the only relevant scale which governs CPP GMR is the spin-diffusion length: once the overall thickness of a magnetic multilayer is shorter than the spin-diffusion length, the series-resistor model becomes valid, in which there are no relevant lengths except the layer thicknesses. It appears, however, that if the laye thicknesses are less than or comparable to the mean free path the interface resistance depends on the layer thicknesses making the series-resistor model inapplicable. The mean free path becomes, therefore, an additional scale, which controls CPP GMR. The application of the series-resistor model is not in general valid for analyzing experimental results, even when the spin-diffusion length is infinite. On the other hand, deviations from the series-resistor model do not necessarily imply that spin-flip scattering processes cause these deviations. Thus, one has to re-examine the parameter obtained from experimental data that have been modeled by the series-resistor model. Comparison of experimental results on CPP GMR with calculations based on accurate multiband models is greatly desirable.

An interesting issue, which has not been reflected in this article, is spin transport through a domain wall in thin films and magnetic nanowires. ${ }^{223-229}$ The magnetic configuration in the system which consists of two ferromagnetic domains separated by a domain wall closely resembles that of a GMR magnetic trilayer: the two regions of oppositely pointing magnetizations are separated by a spacer region, i.e. by the domain wall. The magnetoresistive effect associated with the domain wall in bulk ferromagnets is relatively small due to the large thickness of the domain wall, which is of the order of $1 \mu \mathrm{m}$. Although the domain wall width can be reduced in thin films the magnetoresistive effect is still small (less than $1 \%^{223}$ ). Nevertheless, the domain wall magnetoresistance is interesting from the fundamental point of view where there is still some controversy in our understanding of this phenomenon. ${ }^{227}$ In addition, the domain wall can be made very abrupt in nanocontacts, ${ }^{230}$ which gives the opportunity to observe strongly enhanced magnetoresistance ${ }^{231}$ and spin-dependent conductance quantization. ${ }^{232-234}$

Spin valves containing an antiferromagnetic oxide layer, such as $\mathrm{NiO}$, for exchange biasing and a noble metal overlayer are of increasing interest. This is due to the enhanced values of GMR which can be obtained in these spin valves and which makes them attractive for applications. These enhanced values are explained by the specular scattering of the electrons from the ferromagnet/oxide and ferromagnet/noble metal interfaces. If fully specular reflection on both sides of the FM/NM/FM trilayer is created, the physical situation becomes similar to that of a FM/NM multilayer, resulting in a large gain in the GMR. Unfortunately, the structural quality of the trilayers is normally not as good as the corresponding multilayers, which effects negatively GMR in the spin valves. Recent experimental studies show that the mechanism of specular scattering from ferromagnet/noble metal interfaces does not explain all the available experimental results. ${ }^{94}$ Other effects seem to be important and require further investigations both in theory and in experiment. In addition, very recently the enhancement of GMR was found in spin valve structuress containing nano-oxide layers in the pinned or free ferromagnetic layers. ${ }^{235}$ The mechanism of this enhancement is not completely understood and requires further research.

From the point of view of applications, improvement in the structural integrity of the spin valves is crucial. The microstructure affects strongly not only the transport properties but also the magnetic characteristics. ${ }^{236}$ The correlation between the microstructure and micromagnetics is a challenging issue, which requires further investigations. In particular, we need to study the influence of defects on the creation of domain walls in the process of switching and the magnetization ripple in nanoscale films. The mechanisms of exchange biasing and the search for antiferromagnetic compounds which are suitable as exchange biasing materials are other important issues for the applications of spin valves. ${ }^{45}$

Finally, although magnetoelectronics applications based on magnetotransport phenomena distinct from GMR, such as tunneling magnetoresistance or spin injection into semiconductors, have recently started to attract more and more attention ${ }^{237}$ the physics of GMR in metallic layered structures is so multifaceted that it will undoubtedly remain the subject of great interest in the future.

\section{ACKNOWLEDGEMENTS}

The authors' interest in giant magnetoresistance began in 1995 through a collaborative research program with Hewlett-Packard Laboratories in Palo Alto. We gratefully acknowledge the stimulation provided by Tom Anthony, Jim Brug, Janice Nickel and Chuck Morehouse at Hewlett-Packard during the past six years. We also thank Bill Bailey, Jack Bass, Bill Butler, Sergey Demokritov, Bernard 
Dieny, David Edwards, Peter Grünberg, Paul Kelly, Josef Kudrnovsky, Colin Lambert, Peter Levy, Chris Marrows, George Mathon, Ingrid Mertig, Stuart Parkin, Amanda Petford-Long, Myron Salamon, Ivan Schuller, Jing Shi, John Slonczewski, Tchavdar Todorov, Anatoly Vedyayev, Pete Weinberger, Robert White, and Shufeng Zhang for helpful discussions on particular issues which have been considered in this review. We are especially grateful to Bryan Hickey and Bill Egelhoff for providing us with experimental results before publication and for very useful discussions. The computations at Oxford were performed on the HP Convex Exemplar computer in the Materials Modelling Laboratory at the Department of Materials, University of Oxford, which was funded jointly by Hewlett-Packard and the Higher Education Funding Council of England.

\section{References}

M.N.Baibich, J.M.Broto, A.Fert, F.Nguyen Van Dau, F.Petroff, P.Eitenne, G.Greuzet, A.Friederich, and J.Chazelas, Phys.Rev.Lett. 61, 2472 (1988)

and W.Zinn, Phys.Rev.B 39, 4828 (1989)

B. Brodsky, and H.Sowers, Phys.Rev.Lett. 57, 2442 (1986)

Phys.Rev.Lett. 64, 2304 (1990)

J.Barnas, A.Fuss, R.E.Camley, P.Grünberg, and W.Zinn, Phys.Rev.B 42, 8110 (1990)

(1991)

S. S.P.Parkin, B.A.Gurney, D.R.Wilhoit, and D.Mauri, Phys.Rev.B 43, 1297 (1991).

A.Berkowitz, J.R.Mitchell, M.J.Carey, A.P.Young, S.Zhang, F.E.Spada, F.T.Parker, A.Hutten, and G.Thomas,

Phys.Rev.Lett. 68, 3745 (1992).

1 Tesla is equal to $10^{4}$ Gauss $\left(1 \mathrm{~T}=10^{4} \mathrm{G}\right)$

1. J.M.D.Coey, M.Viret, and S. von Molnar, Adv.Phys. 48, 167 (1999).

J.S.Moodera, J.Nassar, and G.Mathon, Ann.Rev.Mater.Sci. 29, 381 (1999); P.M.Levy and S.Zhang, Cur.Opin in Sol.State\&Mat.Sci. 4, 223 (1999).

13. W.P.Pratt, S.-F.Lee, J.M.Slaughter, R.Loloee, P.A.Schroeder, and J.Baas, Phys.Rev.Lett. 66, 3060 (1991).

14. M.A.M.Gijs, S.K.J.Lenczowski, and J.B.Giesbers, Phys.Rev.Lett. 70, 3343 (1993).

15. T.Ono and T.Shinjo, J.Phys.Soc.Jpn. 64, 363 (1995),

R.J.M. van de Veerdonk, S.K.J.Lenczowski, and R.M.J. van Gansewinkel, Appl.Phys.Lett. 66, 1839 (1995).

18. L. Piraux, J.P.Meier, B.Doudin, and J.-Ph.Ansermet, Appl.Phys.Lett. 65, 3019 (1jegta, and A. Fert, Appl.Phys.Lett. $65,2484(1994)$

19. A.Fert and P.Bruno in Ultrathin Magnetic structures II: An Introduction to the Electronic, Magnetic and Structural Properties, ed. by J.A.C.Bland and B.Heinrich (Berlin: Springer, 1994), p.82.

20. P.M.Levy, in Solid State Physics, ed. by H.Ehrenreich and D.Turnbull, vol.47 (Boston, London: Acad. Press, 1994), p.367.

B.Dieny, J.Magn.Mag.Mat. 136, 335 (1994)

22. M.A.M.Gijs and G.E.W.Bauer, Adv.Phys. 46, 285 (1997).

23. J.-Ph.Ansermet, J.Phys.:Cond.Mat. 10, 6027 (1998)

24. J.Bass and W.P.Pratt Jr., J.Magn.Magn.Mat. 200, 274 (1999).

25. A.Fert and L.Piraux, J.Magn.Magn.Mat. 200, 338 (1999)

26. M.A.M.Gijs, in Magnetic Multilayers and Giant Magnetoresistance: Fundamentals and Industrial Applications, ed. by U.Hartmann (Berlin: Springer, 2000), p.130

P.Coehoorn, in Magnetic Multilayers and Giant Magnetoresistance: Fundamentals and Industrial Applications, ed. by U.Hartmann (Berlin: Springer, 2000), p.65.

28. A.Barthelemy, A.Fert, and F.Petroff, in Handbook of Magnetic Materials, edited by K.H.J.Buschow (Amsterdam Elsevier, 1999), p.1.

Royal Soc. 156, 368 (1936); N.F.Mott, Adv.Phys. 13, 325 (1964)

30. N.W.Ashcroft and N.D.Mermin, Solid State Physics (Philadelphia: Saunders College, 1976).

N.W.Ashcroft and N.D.Mermin, Solid State Physics
J.W.F.Dorleijn, in Philips Res.Rep. 31 (1976) p.287

I.A.Campbell and A.Fert, in Ferromagnetic Materials, edited by E.P.Wohlfarth (Amsterdam: North Holland, 1982), p.769.

p.Prog.Phys, 62, 237 (1999)

34. E.C.Stoner, Proc. Royal Soc. A165, $372(1938)$
5. DM Edwards, R B Muniz and J Mathon, IEEE Trans Magn 3, 4941 (1991).

36. J.Mathon, Contemp.Phys. 32, 143 (1991)

Note that this statement is correct only in the absence of potential steps at the interfaces. This point will be discussed in section VI.

S.S.P.Parkin, ZG.Li, and D.J.Smith, Appl.Phys.Lett. 58, 2710 (1991).

39. R Schad, CD Potter, P Beliën, G Verbanck, JDekoster, G Langouche, V V Moshchalkov, and Y Bruynseraede JMagn Magn Mat 148, 331 (1995); R Schad, CD Potter, P.Beliën, G.Verbanck, V V Moshchalkov, and Y.Bruynseraede, Appl. Phys.Lett. 64, 3500 (1994).

. JM.Gerge, L.G. Perein, A.Bathelemy, F.Petrof,

LB.Steren, J.L.Duvail, A.Fert, R.Loloee, P.Holody, an P.A.Schroeder, Phys.Rev.Lett. 72, 408 (1994).

41. S.Zhang and P.M.Levy, J.Appl.Phys. 69, 4786 (1991)

43. H.E.Camblong, S.Zhang, and P.M.Levy, Phys.Rev.B 47, 4735 (1993).

44. S.S.P.Parkin, R.Bharda, and K.P. Roche, Phys.Rev.Lett. 66, 2152 (1991).

5. J.Nogues and I.K.Schuller, J.Magn.Magn.Mater. 192, 203 (1999)

S.Araki, J.Appl.Phys. 73, 3910 (1993).

B.Rodmacq, M.Vaezzadeh, B.George, and Ph.Mangin, J.Magn.Magn.Mat. 121, 213 (1993).

H.Sato, T.Matsudai, W.Abdul-Razzaq, C.Fierz, and P.A.Schroeder, J.Phys..Cond.Mat. 6, 6151 (1994)

R.Takatani, T.Dei, and Y.Sugita, J.Appl.Phys. 73, 6375 (1993)

50. B.Rodmacq, G.Palumbo, and Ph.Gerard, J.Magn.Magn.Mat. 118, L11 (1993)

51. S.S.P.Parkin, R.F.S.Farrow, R.F.Marks, A.Cebollada, G.R.Harp, and R.J.Savoy, Phys.Rev.Lett. 72, 3718 (1994),

52. M.E.Brubaker, J.E.Mattson, C.H.Sowers, and S.D.Bader, Appl.Phys.Lett. 58, 2306 (1991)

53. K.Shintaki, Y.Daitoh, and T.Shinjo, Phys.Rev.B 17,14584 (1993)

54. Q.Y.Jin, M.Lu, Q.S.Bie, Y.B.Xu, H.R.Zhai, and Y.H.Shen, J.Magn.Magn.Mater, 140-144, 565 (1995)

55. K.H.Yanagihara, K.Pettit, M.B.Salamon, E.Kita, and S.S.P.Parkin, J.Appl.Phys. 81, 5197 (1997)

56. B.Dieny, V.S.Speriosu, B.A.Gurney, S.S.P.Parkin, D.R.Wilhoit, K.P.Roche, S.Metin, D.T.Peterson, and S.Nadim J.Magn.Magn.Mat. 93, 101 (1991)

57. T.L.Monchesky, B.Heinrich, R.Urban, K.Myrtle, M.Klaua, and J.Kirschner, Phys.Rev.B 60, 10242 (1999).

58. P.Coehoorn, J.Magn.Magn.Mat. 121, 432 (1993).
59. J.F.Bobo, K.Bessho, F.B.Mancoff, P.R.Johnson, M.Kautzky, R.L.White, and B.M.Clemens, Mater.Res.Soc.Proc 475, 21 (1997); M.Kautzky, F.B.Mancoff, J.F.Bobo, P.R.Johnson, R.L.White, and B.M.Clemens, J. Appl.Phys. 81, 4026 (1997).

60. J.A.Caballero, Y.D.Park, J.R.Childress, J.Bass, W.C.Chiang, A.C.Reilly, W.P.Pratt, and F.Petroff, J.Vac.Sci.Technol. A 16, 1801 (1998)

61. C.Hordequin, J.P.Nozieres, and J.Pierre, J.Magn.Magn.Mat. 183, 225 (1998); D.Ristoiu, J.P.Nozieres, an L.Ranno, J.Magn.Magn.Mat. 219, 97 (2000).

62. R.A. de Groot and F.M.Mueller, P.G. van Engen, K.H.J.Buschow, Phys.Rev.Lett. 50, 2024 (1983),

64. B.Dieny, V.S.Speriosu, S.Metin, S.S.P.Parkin, B.A.Gurney, P.Baumgart, and D.R.Wilhoit, J.Appl.Phys. 69, 4774 (1991).

S.S.P.Parkin, A.Modak, and D.J.Smith, Phys.Rev.B 47, 9136 (1993)

66. B.Dieny, P.Humbert, V.S.Speriosu, S.Metin, B.A.Gurney, P.Baumgart, and H.Lefakis, Phys.Rev. B 45,806 (1992). 67. M.Sato, S.Ishio, and T.Miyazaki, J.Magn.Magn.Mat. 126, 460 (1993).

68. B. A. Gurney, V.S.Speriosu, J P. Nozieres, H Lefakis, D.R. Wilhoit, and O.U.Need, Phys Rev Lett. 71, 4023 (1993).

69. E.F.Fullerton, D.M.Kelly, J.Guimpel, I.K.Schuller, and Y.Bruynseraede, Phys.Rev.Lett. 68, 859 (1992)

70. R Schad, P.Beliën, G Verbanck, V V Moshchalkov, Y Bruynseraede, H.E.Fischer, S.Lefebvre, and B Bessiere, Phys.Rev.B 59, 1242 (1999)

1. R.Schad, P.Beliën, G.Verbanck, C.D.Potter, H.E.Fischer, S.Lefebvre, and B.Bessiere, V.V.Moshchalkov, and Y.Bruynseraede, Phys.Rev.B 57, 13692 (1998)

72. H.Kano, K.Kagawa, A.Suzuki, A.Okabe, K.Hayashi, and K.Asoa, Appl.Phys.Lett. 63, 2839 (1993).

73. M.Suzuki and Y.Taga, Phys.Rev.B 52, 361 (1995).

74. B.A.Gurney, P.Baumgart, D.R.Wilhoit, B.Dieny, and V.S.Speriosu, J.Appl.Phys. 70, 5867 (1991); P.Baumgart, B.A.Gurney, D.R.Wilhoit, T.Nguyen B.Dieny, and V.S.Speriosu, J.Appl.Phys. 69, 4792 (1991).

75. B.L.Johnson and R.E.Camley, Phys.Rev.B 44, 9997 (1991)

76. C.H.Marrows and B.J.Hickey, unpublished.

78. S.S.P.Parkin, Phys.Rev.Lett. 71, 1641 (1993). Soc.Jpn. 61, 1149 (1992).

79. V.S.Speriosu, J.P.Nozieres, B.A.Gurney, B.Dieny, T.C.Huang, H.Lefakis, Phys.Rev.B 47, 11579 (1993). 
80. A.B.Oparin, D.M.C.Nicholson, X.G.Zhang, W.H.Butler, W.A.Shelton, G.M.Stocks, Y.Wang, J.Appl.Phys. 85 $4548(1999)$.

81. C.Vouille, A.Barthelemy, F.E.Mpondo, A.Fert, P.A.Schroeder, S.Y.Hsu, A.Reilly, and R.Loloee, Phys.Rev.B 60 6710 (1999); J.Appl.Phys. 81, 4573 (1997)

82. S.Y.Hsu, A.Barthelemy, P.Holody, R.Loloee, P.A.Schroeder, and A.Fert, Phys.Rev.Lett. 78, 2652 (1997).

83. T.S.Plaskett and T.R.McGuire, J.Appl.Phys, 73, 6378 (1993).

84. K.Fuchs, Proc. Cambridge Phil Soc 34, 100 (1938)

85. E.H.Sondheimer, Adv Phys. 1 , 1 (1952).

86. P.P.Freitas, J.L.Leal, T.S.Plaskett, L.V.Melo, and J.C.Soares, J.Appl.Phys. 75, 6480 (1994).

87. T.C.Anthony, J.A.Brug, and S.Zhang, IEEE Trans.Magn. 30, 3819 (1994)

88. H.Swagten, G.J.Strijkers, P.J.H.Bloemen, M.M.H.Willekens, and W.J.M. de Jonge, Phys.Rev.B. 53, 9108 (1996)

W.F.Egelhoff, T.Ha, R.D.K.Misra, Y.Kadmon, J.Nir, C.J.Powell, M.D.Stiles, R.D.McMichael, C.L.Lin, J.M.Sivertsen, J.H.Judy, K.Takano, A.E.Berkowitz, T.C.Anthony, and J.A.Brug, J.Appl.Phys.78, 273 (1995).

90. W.F.Egelhoff, P.J.Chen, C.J.Powell, M.D.Stiles, R.D.McMichael, C.L.Lin, J.M.Sivertsen, J.H.Judy, K.Takano, A.E.Berkowitz, T.C.Anthony, and J.A.Brug, J.Appl.Phys. 79, 5277 (1996).

91. W.F.Egelhoff, P.J.Chen, C.J.Powell, M.D.Stiles, R.D.McMichael, J.H.Judy, K.Takano, and A.E.Berkowitz J.Appl.Phys. 82, 6142 (1997).

93. W.F.Egelhoff, P.J.Chen, C.J.Powell, M.D.Stiles, R.D.McMichael, J.H.Judy, K.Takano, A.E.Berkowitz, an J.M.Daughton, IEEE Trans.Magn. 33, 3580 (1997).

W.F.Egelhoff, private communication.

95. F.Petroff, A.Barthelemy, A.Fert, P.Etienne, and S.Lequien, J.Magn.Magn.Mat. 93, 95 (1991),

96. W.Oepts, M.A.M.Gijs, A.Reinders, and R.M.Jungblut, R.M.J. van Gansewinkel, and W.J.M. de Jonge, Phys.Rev.B 53, $14024(1996)$

A.Chaiken, G.A.Prinz, and J.J.Krebs, J.Appl.Phys. 67, 4892 (1990).

9. A.Vedyayev, B.Dieny, N.Ryzhanova, J.B.Genin, and C.Cowache, Europhys.Lett. 25, 465 (1994)

L.Steren, R.Morel, A.Barhomy, F.Petroff, A.Fert, P.Holody, R.Loloee, and P.A.Schroeder, J.Magn.Magn.M 140-144, 495 (1995); L.B.Steren, A.Barthelemy, J.L.Duvail, A.Fert, R.Morel, F.Petroff, P.Holody, R.Loloee, an P.A.Schroeder, Phys.Rev.B 51, 292 (1995)

101. C.Blaas, P.Weinberger, L.Szunyogh, J.Kudrnovsky, V.Drchal, P.M.Levy, and C.Sommers, Eur.Phys.J. 9, 245

102. P.Dauguet, P.Grandit, J.Chaussy, S.F.Lee, A.Fert, and P.Holody, Phys.Rev.B 54, 1083 (1996).

103. J.M.Ziman, Principles of the Theory of Solids (London: Cambridge Univ. Press, 1972).

8009 (1992)

.

105. R.C.C

108. A.Batelon and A.Fet, Phy Revi 43,13124 (1991).

109. B. Bieny, Europhys Lett 17, 261 (1992)

110. B.Dieny, V.S.Speriosu, J.-P Nozieres, B.A.Gurney, A. Vedyaev, and N.Ryzhanova, in Magnetism and Structure Systems of Reduced Dimensions, ed. by R.F.C.Farrow et al. (New York: Plenum, 1993)

11. W.E.Bailey, PhD Thesis (Stanford University, 2000).

112. W.E.Bailey, S.W.Wang, and E.Y.Tsymbal, Phys.Rev.B 61, 1330 (2000).
113. W.E.Bailey, S.W.Wang, and E.Y.Tsymbal, J.Appl.Phys. 87, 5185 (2000).

114. R.Q.Hood and L.M.Falicov, Phys.Rev.B 46, 8287 (1992); R.Q.Hood, L.M.Falicov, and D.R.Penn, Phys.Rev.B 49, 368 (1994).

15. N.Trivedy and N.W.Ashcroft, Phys.Rev.B 38, 12298 (1988).

116. J.M.Ziman, Electrons and Phonons (London: Oxford Univ. Press, 1972).

117. Z.Tesanovic, M.V.Jaric, and S.Maekawa, Phys. Rev. Lett. 57, 2760 (1986)

18. R.Kubo, J.Phys.Soc.Jpn. 12, 570 (1957)

19. R.Landauer, IBM J.Res.Dev. 1, 233 (1957); IBM J.Res.Dev. 32, 306 (1998).

20. L.V.Keldysh, Sov.Phys.JETP 20, 1018 (1965)

21. G.D.Mahan, Many-particle physics (New York, London: Plenum Press, 1990).

122. S.Datta, Electronic transport in mesoscopic systems (Cambridge: Univ. Press, 1995).

123. J.Rammer and H.Smith, Rev.Mod.Phys. 58, 3234 (1986)

24. D.A.G

125. S.Doniach and E.H.Sondheimer, Green's functions for Solid State Physicists (London: River Edge, 1998)

126. H.Ehrenreich and L.M.Schwartz, in Solid State Physics, ed. by H.Ehrenreich, F.Seitz, and D.Turnbull, vol.31 (New York, London: Academic Press, 1976), p.149.
127. M Johnson and R.H.Silsbee, Phys.Rev.Lett. 55, 1790 (1985); Phys.Rev.B 35, 4959 (1987); Phys.Rev.B 37, 5312 (1988).

128. P.M.Levy, S.Zhang, and A.Fert, Phys.Rev.Lett. 65, 1643 (1990); S.Zhang, P.M.Levy, and A.Fert, Phys.Rev.B 45, 8689 (1992).

H.E.Camblong and P.M.Levy, Phys.Rev.Lett. 69, 2835 (1992); J.Appl.Phys. 73, 5533 (1993).

130. H.E.Camblong, Phys.Rev.B 51, 1855 (1995).

131. B.Dieny, A.Vedyayev, and N.Ryzhanova, J.Magn.Magn.Mat. 121, 366 (1993)

132. A Vedyayev, B Dieny, and N Ryzhanova, Europhys Lett. 19, 329 (1992).

133. X.-G.Zhang and W.H.Butler, Phys.Rev.B 51, 10085 (1995)

134. S.Zhang and P.M.Levy, in Magnetic Ultrathin Films, Multilavers and Surfaces, Interfaces and Characterization, MRS Symp.Proc. No.313 (Pittsburgh: MRS Society, 1993), p.53.

135. B.R.Bulka and J.Barnas, Phys.Rev.B 51, 6348 (1995).

136. A.Vedyayev, C.Cowache, N.Ryzhanova, and B.Dieny, J.Phys.:Cond.Mat. 5, 8289 (1993); A.Vedyayev, M.Chshiev, and B.Dieny, J.Magn.Magn.Mat. 184, 145 (1998)

37. J.Barnas and Y. Bruynseraede, Phys.Rev.B 53, 5449 (1996). W.A.Harrison, Electronic Structure and the Properties of Solids (New
and structure of molecules and solids (Oxford: Clarendon Press, 1995).

P.A.Lee and D.S.Fisher, Phys.Rev.Lett. 47, 882 (1981).

140. D.S.Fisher, and P.A.Lee, Phys.Rev.B Lett. 23, 6851 (1981).

141. T.N.Todorov, Phys. Rev. B 54, 5801 (1996).

142. J.Pollmann and S.T.Pantelides, Phys.Rev.B 18, 5524 (1978)

143. Y.Asano, A.Oguri, and A.Maekawa Phys.Rev.B 48, 6192 (1993).

144. H.Itoh, J.Inoue, and S.Maekawa, Phys.Rev.B 51, 342 (1995).

145. B.Velicky, Phys.Rev. 184, 614 (1969).

146. T.N.Todorov, E.Y.Tsymbal, and D.G.Pettifor, Phys.Rev.B 54, R12685 (1996)

147. H.Itoh, J.Inoue, and S.Maekawa, Phys.Rev.B 47, 5809 (1993).

48. J.Callaway and N.H.March, in Solid State Physics, ed. by H.Ehrenreich and D.Turnbull, vol.38 (Orlando: Acad. Press, 1984), p.136

149. P.Hohenberg and W.Kohn, Phys.Rev.B 136, 864 (1964).

150. W.Kohn and L.J.Sham, Phys.Rev.A 140, 1133 (1965)

2064 (1971); U. von Barth and L.Hedin, J.Phys.C 5, 1629 (1972).

52. We note, however, that the LSDA is deficient in predicting the correct ground-state crystal structure of iron. See, e.g., C.S.Wang, B.M.Klein, and H.Krakauer, Phys.Rev.Lett. 54, 1852 (1985).

153. Strictly speaking the single particle functions $\psi_{i}(\mathbf{k})$ introduced by Kohn and Sham have no significance beyon constructing the charge density in equation (V.1) and the eigenvalues $E_{i}$ are not equivalent to the quasi-particle excitation energies which are required for calculating transport properties. This distinction is, however, relevant only beyond the local spin-density approximation.

, ed by H.Enreich, F.Seitz, and D.Turnbull, vol.26 (New York, London Acad. Press, 1971), p.103; T.L.Loucks, Augmented Plane Wave Method (New York: Benjamin, 1967).

155. J.Korringa, Physica 13, 392 (1947); W.Kohn and N.Rostoker, Phys.Rev. 94, 1111 (1954); see also B.Segall and F.S.Ham, in Methods in Computational Physics, vol.8 (New York: Academic, 1968), chap.7.

156. O.K.Andersen, Phys.Rev.B 12, 3060 (1975); H.L.Skriver, The LMTO method (New York: Springer, 1984)

157. J.C.Slater and G.F.Koster, Phys.Rev. 94, 1498 (1954).

158. D.A.Papaconstantopoulos, Handbook of the Band Structure of Elemental Solids (New York: Plenum, 1986).

5.P.Sutton, M.W.Finnis, D.G.Pettifor and Y.Ohta, J.Phys.C 21, 35 (1988).

60. O.K.Andersen and O.Jepsen, Phys.Rev.Letters 53, 2571 (1984); O.K.Andersen, O.Jepsen, and M.Sob, Electronic Band Structure and its Applications, ed. by M.Yussouff (Berlin: Springer, 1987).

161. Yu.V.Sharvin, Sov.Phys.JETP 21, 665 (1965).

162. K.M.Schep, P.J.Kelly, and G.E.W.Bauer, Phys.Rev.Lett. 74, 568 (1995).

163. K.M.Schep, P.J.Kelly, and G.E.W.Bauer, Phys.Rev.B 57, 8907 (1998).

164. J.Mathon, M.Villeret, and H.Itoh, Phys.Rev.B 52, R6983 (1995).

165. P.Bruno and C.Chappert, Phys.Rev.Lett. 67, 1602 (1991); Phys.Rev.B 46, 261 (1992).

166. J.Mathon, A.Umerski, and M.Villeret, Phys.Rev.B 55, 14378 (1997)

67. T.Oguchi, J.Magn.Magn.Mat. 126, 519 (1993); Mat.Science and Eng. 31, 111 (1995).

68. P.Zahn, I.Mertig, M.Richer, and H.Eschrig, Phys.Rev.Lett. 75, 2996 (1995)

. S.Blügel, R.Zeller, and P.H.Dederichs, Phys.Rev.B 40, 8203 (1989); I.Mertig, R.Zeller, and P.H.Dederichs, Phys.Rev.B 47, 16178 (1993)

170. J.Binder, P.Zahn, and I.Mertig, J.Appl.Phys. 87, 5182 (2000). 
171. W.H.Butler, J.M.MacLaren, and X.-G.Zhang, in Magnetic Ultrathin Films, Multilayers and Surfaces, Interfaces and Characterization, MRS Symp.Proc. No.313 (Pittsburgh: MRS Society, 1993), p.59.

173. W.H.Butler, X.-G.Zhang, D.M.C.Nicholson, and J.M.MacLaren, J.Magn.Magn.Mat. 151, 354 (1995).

174. A. Vernes, H.Ebert, and J.Banhart, Comp Mater Sci 10, 221 (1998)

175. P. Zahn, J.Binder, IMertig. R Zeller, and P.H.Dederichs, Phys Rev.Lett. 80, 4309 (1998).

176. J.Binder, P.Zahn, and I.Mertig unpublished.

177. P.Bayle-Guillemaud, A.K.Petford-Long, T.C.Anthony, and J.A.Brug, IEEE Trans.Magn. 32, 4627 (1996).

178. T.G.S.M.Rijks, R.L.H.Sour, D.G.Neerinck, A.E.M. de Veirman, R.Coehoorn, J.C.S.Kools, M.F.Gillies, and W.J.M. de Jonge, IEEE Trans.Magn. 31, 3865 (1995)

79. E.Y.Tsymbal and D.G.Pettifor, J.Phys.: Cond.Mat. 8, L569 (1996); Phys.Rev.B 54, 15314 (1996); J.Appl.Phys. 81, 4579 (1997)

180. T.C.Schulthess, W.H.Butler, X.-G.Zhang, D.M.C.Nicholson, and J.M.MacLaren, Phys.Rev.B 56, 8970 (1997).

181. D.J.Monsma, J.C.Lodder, T.J.A.Popma, and B.Dieny, Phys.Rev.Lett. 74, 5260 (1995).

182. R.Jansen, P.S.A.Kumar, O.M.J. van't Erve, R.Vlutters, P. de Haan, and J.C.Lodder, Phys. Rev. Lett. 85, 3277 (2000).

183. E.Y.Tsymbal and D.G.Pettifor, J.Magn.Magn.Mat. 202, $163(1999)$

184. E.Y.Tsymbal, D.G.Pettifor, J.Shi, and M.B.Salamon, Phys.Rev.B 59, 8371 (1999).

85. J.Sakurai, M.Horie, S.Araki, H.Yamamoto, and T.Shinjo, J.Phys.Soc.Jpn. 60, 2522 (1991); M.J.Conover, M.B.Brodsky, J.E.Mattson, C.H.Sowers, and S.D.Bader, J.Magn.Magn.Mat. 102, L5 (1991); L.Piraux, A.Fert, P.A.Schroeder, R.Loloee, and P.Etienne, J.Magn.Magn.Mat. 110, L247 (1992)

186. J.Shi, R.C.Yu, S.S.P.Parkin, and M.B.Salamon, J.Appl.Phys. 73, 5524 (1993); J.Shi, S.S.P.Parkin, L.Xing, an M.B.Salamon, J.Magn.Magn.Mat. 125, L251 (1993), K.Ni and T.Shinjo, J. Phys. Soc. Jpn. 63, 2685 (1994); J.Sakurai, K.Hasegawa, K.Shintaku, and T.Shinjo, J.Phys.Soc.Jpn. 64, 3897 (1995); J.Shi, K.Pettit, E.Kita, S.S.P.Parkin, R.Nakatani, and M.B.Salamon, Phys.Rev. B $\mathbf{5 4 ,} 15273$ (1996).

187. N.F.Mott and H.Jones, The Theory of the Properties of Metals and Alloys (Oxford: Clarendon Press, 1936).

88. W.H.Butler, X.-G.Zhang, D.M.C.Nicholson, and J.M.MacLaren, Phys.Rev.B 52, 13399 (1995)

189. M.D.Stiles, J.Appl.Phys. 79, 5805 (1996)

190. W.H.Butler, X.-G.Zhang, D.M.C.Nicholson, T.C.Schulthess, and J.M.MacLaren, Phys.Rev. Lett. 76, 3216 (1996).

191. M.D.Stiles and D.R.Hamann, Phys.Rev.B 38, 2021(1988); Phys.Rev.B 40, 1349 (1989).

192. DT Dekadjevi, P.A.Ryan, B J Hickey, B.DFFlhore, and B K Tanner, unpublished.

193. W.H.Butler, X.-G.Zhang, T.C.Schulthess, D.M.C.Nicholson, J.M.MacLaren, V.S.Speriosu, and B.A.Gurney, Phys.Rev. B 56, 14574 (1997).

(1999).

195. Y.Wang, G.M.Stocks, W.A.Shelton, D.M.C.Nicholson, Z.Szotek, and W.M.Temmerman, Phys.Rev.Lett. 75, 2867 (1995).

196. W.H.Butler, X.G.Zhang, T.C.Schulthess, D.M.C.Nicholson, A.B.Oparin, and J.M.MacLaren, J.Appl.Phys. 85 5834 (1999).

197. S.-F.Lee, W.P.Pratt, R.Loloee, P.A.Schroeder, and J.Baas, Phys.Rev.B 46, 548 (1992); S.-F.Lee, W.P.Pratt, Q.Yang, P.Holody, R.Loloee, P.A.Schroeder, and J.Baas, J.Magn. Magn.Mat. 118, L1 (1993).

198. S.-F.Lee, Q.Yang, P.Holody, R.Loloee, J.H.Hetherington, S.Mahmood, B.Ikegami, K.Vigen, L.L.Henry, P.A.Schroeder, W.P.Pratt, and J.Baas, Phys.Rev.B 52, 15426 (1995)

199. N.J.List, W.P.Pratt, M.A.Howson, J.Xu, M.J.Walker, and D.Greig, J.Magn.Magn.Mat. 148, 342 (1995).

200. L.Piraux, S.Dubois, and A.Fert, J.Magn.Magn.Mat. 159, L287 (1996).

201. B.Doudin, A.Blondel, and J.-Ph.Ansermet, J.Appl.Phys. 79, $6090(1996)$

202. J.Barnas and A.Fert, Phys. Rev. B 49, 12835 (1994); J.Magn.Magn.Mat. 136, 260 (1994).

203. S.Zhang and P.M.Levy, Phys. Rev. B 57, 5336 (1998).

204. K.M.Schep, J.B.A.N. van Hoof, P.J.Kelly, G.E.W.Bauer, and J.E.Inglesfield, Phys.Rev.B 57, 8907 (1998)

205. J.Chen, T.-S.Choy, and S.Hershfield, J.Appl.Phys. 85, 4551 (1999).

206. M.D.Stiles and D.R.Penn, Phys.Rev.B 61, 3200 (2000).

207. E.Y.Tsymbal and D.G.Pettifor, Phys.Rev.B 61, 506 (2000)

208. E.Y.Tsymbal, Phys.Rev.B 62, R3608 (2000).

209. W.H.Butler, X.G.Zhang, and J.M.MacLaren J.Appl.Phys. 87, 5173 (2000); Supercond. 13, 221 (2000).

210. K.Xia, P.J.Kelly, G.E.W.Bauer, I.Turek, J.Kudrnovsky, and V.Drchal, Phys.Rev.B, in press

211. J.Kudrnovsky, V.Drchal, I.Turek, C.Blaas, P.Weinberger, and P.Bruno, Czech. J. Phys. 49, 1583 (1999); Surf.Sci. 454-456, 918 (2000); Phys.Rev.B 62, 15084 (2000).

212. A.Shpiro and P.M.Levy, Phys. Rev. B 63, 014419 (2001).

13. D.Bozec, M.A.Howson, B.J.Hickey, S.Shatz, N.Wiser, E.Y.Tsymbal, and D.G.Pettifor, Phys.Rev.Lett. 85, 1314 (2000).
214. J.Kudrnovsky, V.Drchal, I.Turek, P.Bruno, and P.Weinberger, unpublished.

215. S. Sanvito, C J Lambert, and J.H Jefferson, Phys.Rev B 61, 14225 (2000).

216. J.Mathon, Phys.Rev.B 54, 55 (1996); Phys.Rev.B 55, 960 (1997)

17. S.Sanvito, C.J.Lambert, and J.H.Jefferson, Phys.Rev.B 60, 7385 (1999).

18. T.Valet and A.Fert, Phys. Rev. B 48, 7099 (1993).

219. Q.Yang, P.Holody, S.-F.Lee, L.L.Henry, R.Loloee, P.A.Schroeder, W.P.Pratt, and J.Bass, Phys. Rev. Lett. 72 $3274(1994)$

220. S.D.Steenwyk, S.Y.Hsu, R.Loloee, J.Baas, and W.P.Pratt, J. Magn. Magn. Mat. 170, L1 (1997).

221. S.Dubois, L.Piraux, J.M.George, K.Ounadjela, J.L.Duvail, and A.Fert, Phys.Rev.B 60, 477 (1999).

222. W. Park, D.V.Baxter, S.Steenwyk, I.Moraru, W.P.Pratt, and J.Baas, Phys.Rev.B 62, 1178 (2000).

223. J.F.Gregg, W.Allen, K.Ounadjela, M.Viret, M.Hehn, S.M.Thompson, and J.M.D.Coey, Phys.Rev Lett. 77, 1580 (1996)

224. U.Rüdiger, J.Yu, S.Zhang, A.D.Kent, and S.S.P.Parkin, Phys.Rev.Lett. 80, 5639 (1998); U.Rüdiger, J.Yu, L.Thomas, S.S.P.Parkin, and A.D.Kent, Phys.Rev.B 59, 11914 (1999)

225. P.M.Levy and S.Zhang, Phys.Rev.Lett. 79, 5110 (1997).

226. R.P. van Gorkom, A.Brataas, and G.E.W.Bauer, Phys.Rev.Lett. 83, 4401 (1999); J.B.A.N. van Hoof, K.M.Schep, A.Brataas, G.E.W.Bauer, and P.Kelly, Phys.Rev.B 59, 138 (1999); A.Brataas, G.Tatara, and G.E.W.Bauer, Phys.Rev.B 60, 3406 (1999)

227. Y.B.Xu, C.A.F.Vaz, A.Hirohata, H.T.Leung, C.C.Yao, and J.A.C.Bland, E.Cambril, F.Rousseaux, and H.Launois, Phys.Rev.B 61, R14901 (2000).

228. U.Ebels, A.Radulescu, Y.Henry, L.P.

229. J.-E.Wegrowe, A.Comment, Y.Jaccard, J.-Ph.Ansermet, N.M.Dempsey, and J.-P.Nozieres, Phys.Rev.B 61, 12216 (2000).

230. P.Bruno, Phys.Rev.Lett. 83, 2425 (1999).

231. N.Garcia, M.Munoz, and Y.-W.Zhao, Phys.Rev.Lett. 82, 2923 (1999).

232. H.Oshima and K.Miyano, Appl.Phys.Lett. 73, 2203 (1998)

233. T.Ono, Y.Ooka, H.Miyajima, and Y.Otani, Appl.Phys.Lett. 75, 1622 (1999).

234. H.Imamura, N.Kobaysh, S.Takasashi, and S.Maekawa, Phys.Rev.Lett. 84, 1003 (2000).

235. H.Sakakima, M.Satomi, Y.Sugita, Y.Kawawake, J.Magn.Magn.Mat. 210, L20 (2000); M.F.Gillies, A.E.T.Kuiper, J.Appl.Phys. 88, 5894 (2000)

236. X.Portier and A.K.Petford-Long, J.Phys.D: Appl.Phys. 32, R89 (1999).

237. G.A.Prinz, J.Magn.Magn.Mater. 200, 57 (1999). 